\title{
Uncertainties associated with in situ high-frequency long- term observations of suspended particulate matter concentration using optical and acoustic sensors
}

\author{
Fettweis Michael ${ }^{1,{ }^{*}}$, Riethmüller Rolf $2,{ }^{*}$, Verney Romaric ${ }^{3}$, Becker Marius ${ }^{4}$, Backers Joan ${ }^{1}$, \\ Baeye Matthias ${ }^{1}$, Chapalain Marion ${ }^{3}$, Claeys Styn ${ }^{5}$, Claus Jan ${ }^{6}$, Cox Tom ${ }^{7}$, Deloffre Julien ${ }^{8}$, \\ Depreiter Davy ${ }^{6}$, Druine Flavie ${ }^{8,9}$, Flöser Götz ${ }^{2}$, Grünler Steffen ${ }^{10}$, Jourdin Frédéric ${ }^{11}$, Lafite Robert ${ }^{8}$, \\ Nauw Janine ${ }^{12,13}$, Nechad Bouchra ${ }^{1}$, Röttgers Rüdiger ${ }^{2}$, Sottolichio Aldo ${ }^{14}$, Van Engeland Tom ${ }^{7}$, \\ Vanhaverbeke Wim ${ }^{1}$, Vereecken Hans ${ }^{5}$
}

\author{
${ }^{1}$ Royal Belgian Institute of Natural Sciences, OD Nature, Rue Vautier 29, 1000 Brussels, Belgium \\ 2 Helmholtz-Zentrum Geesthacht, Institute for Coastal Research, Max-Planck-Str. 1, 21502 Geesthacht, \\ Germany \\ 3 IFREMER, Laboratoire DHYSED, CS10070, 29280 Plouzané, France \\ ${ }^{4}$ Christian-Albrechts-Universität, Institute of Geosciences, Otto-Hahn-Platz 1, 24118 Kiel, Germany \\ ${ }^{5}$ Flanders Hydraulics Research, Berchemlei 115, 2140 Antwerp, Belgium \\ 6 IMDC, Van Immerseelstraat 66, 2018 Antwerp, Belgium \\ 7 University of Antwerp, Ecosystem Management Research Group, Universiteitsplein 1C -C.0.32, 2610 \\ Wilrijk, Belgium \\ ${ }^{8}$ Normandie University Rouen, UNIROUEN, UNICAEN, CNRS, M2C, 76000 Rouen, France \\ 9 University of Tours, EA GéoHydrosystèmes continentaux, Parc Grandmont, 37200 Tours, France \\ 10 Federal Waterways Engineering and Research Institute (BAW), Wedeler Landstr. 157, 22559 \\ Hamburg, Germany \\ 11 Service Hydrographique et Océanographique de la Marine (SHOM), 13 rue du Chatellier, 29228 \\ Brest, France \\ 12 Royal Netherlands Institute for Sea Research, Department of Coastal Systems Royal Netherlands \\ Institute for Sea Research, Department of Coastal Systems and Utrecht University, PO Box 59, 1790 \\ AB Den Burg Texel, The Netherlands \\ ${ }^{13}$ Royal Netherlands Institute for Sea Research, Department of Coastal Systems and Utrecht \\ University, PO Box 59, 1790 AB Den Burg Texel, The Netherlands \\ 14 University of Bordeaux, EPOC, UMR5805, 33600, Pessac, France \\ *Corresponding authors : Michael Fettweis, email address : mfettweis@naturalsciences.be ; Rolf \\ Riethmüller, email address : rolf.riethmueller@hzg.de
}

\begin{abstract}
:
Measurement of suspended particulate matter concentration (SPMC) spanning large time and geographical scales have become a matter of growing importance in recent decades. At many places worldwide, complex observation platforms have been installed to capture temporal and spatial variability over scales ranging from $\mathrm{cm}$ (turbulent regimes) to whole basins. Long-term in situ measurements of SPMC involve one or more optical and acoustical sensors and, as the ground truth reference, gravimetric measurements of filtered water samples. The estimation of SPMC from optical and acoustical proxies
\end{abstract}


generally results from the combination of a number of independent calibration measurements, as well as regression or inverse models. Direct or indirect measurements of SPMC are inherently associated with a number of uncertainties along the whole operation chain, the autonomous field deployment, to the analyses necessary for converting the observed proxy values of optical and acoustical signals to SPMC. Controlling uncertainties will become an important issue when the observational input comprises systems of sensors spanning large spatial and temporal scales. This will be especially relevant for detecting trends in the data with unambiguous statistical significance, separating anthropogenic impact from natural variations, or evaluating numerical models over a broad ensemble of different conditions using validated field data.

The aim of the study is to present and discuss the benefits and limitations of using optical and acoustical backscatter sensors to acquire long-term observations of SPMC. Additionally, this study will formulate recommendations on how to best acquire quality-assured SPMC data sets, based on the challenges and uncertainties associated with those long-term observations. The main sources of error as well as the means to quantify and reduce the uncertainties associated with SPMC measurements are also illustrated.

\section{Highlights}

- Errors associated with optical and acoustical sensors for SPMC are quantified. A strict protocol limits the uncertainties. Systematic errors may reach up to $\pm 20 \%$ and errors due to biofouling to $100 \%$ or more. Changes of the inherent particle properties may result in uncertainties up to $200 \%$. A model based on the $\mathrm{R}^{2}$ quantifies the uncertainty of the sensor derived SPMC. Hach-turbidities could be a cheap alternative for sample SPMC.

Keywords : Suspended Particulate Matter, Measurement uncertainty, Regression, Optical and acoustical sensors 


\section{Introduction}

Water clarity is an important parameter to understand marine ecosystems and is mainly controlled by suspended particulate matter concentration (SPMC). To detect variability in SPMC, networks of observational platforms have been installed worldwide using optical and acoustical sensors (e.g. Butman et al., 1979; Grabemann and Krause, 1989 Fettweis et al., 1998; Guézennec et al., 1999; Ganju and Schoelhamer, 2006; Badewien et al., 2009; Palinkas et al., 2010; Garel and Ferrera, 2011; Nauw et al., 2014; Anastasiou et al., 2015; Jalón-Rojas et al., 2015; Many et al., 2016; Druine et al., 2018) as well as sensors or samples that give information on the shape, size and composition of the SPM (e.g. Krivtsov et al., 2008; Cartwright et al., 2009; Gray and Gartner, 2009; Fettweis and Baeye, 2015; van der Hout et al., 2015; Chapalain et al., 2019). In addition to these sensors gravimetric measurements of filtered water samples are generally used as ground truth reference (e.g. Neukermans et al., 2012a). The infrastructure on which the sensors are attached includes fixed (piles, benthic landers, tripods, moored lines and buoys) and moving platforms (vessels, gliders, AUV, ROV, floats) or a combination of both (Baschek et al., 2017), and the SPMC may cover the whole range of coastal environments from oligotrophic to hyperturbid conditions.

Long-term and high frequency data series of SPMC are typically collected indirectly with autonomous sensors that measure either the optical beam attenuation as a percentage of light transmission (Moody et al., 1987; Spinrad et al., 1989; Agrawal and Pottsmith, 2000), the backor sidescatter intensity of light in volt or factory calibrated turbidity units, or the acoustic backscatter in counts or volts (Thorne and Hanes, 2002; Downing, 2006; Rai and Kumar, 2015). The measuring frequency should at least be able to resolve the dominant variations (often the tidal cycle) and might be as high as to record finer scale variations such as turbulence. The time span of long-term measurements should cover variations ranging from lunar to seasonal and inter-annual cycles.

The combination of different sensors measuring in environments with high spatiotemporal variability implicitly demands adapted pre- and post-measurement procedures in order to obtain homogenous data sets. Conversion of the sensor output to physical units (e.g. mass or volume concentration of particles) results from laboratory and field calibrations, as well as data post processing procedures. The whole procedure requires direct measurements in the laboratory using standard turbidity solutions for calibration, and in the field using SPM mass concentrations determined through filtration of water samples. The way the sensor output is transformed to SPMC (in $\mathrm{g} / \mathrm{I}$ ) depends further on the modelling techniques used to relate sensor output to SPMC and is further complicated by the significant variability of the inherent optical (IOP) and acoustical properties (IAP) of the SPM (e.g. Slade et al., 2011; Woźniak et al., 
Version: August 11, 2019;

2011; Sahin et al., 2017). To guarantee quality and repeatability the laboratory procedures and the measuring process need to be documented; from the planning of the measurements to the post processing of the data (Lane et al., 2000; Waldmann et al., 2010; Bolanos et al., 2011; Gil et al., 2016).

Direct or indirect measurements of SPMC are inherently associated with a number of uncertainties along the whole operational chain; from the laboratory work, to system problems due to the autonomous nature of the sensor deployments, to converting the observed proxy values of optical and acoustic signals to SPMC. Guidelines for good practice for fluvial sediment transport measurements can be found in e.g. Rasmussen et al. (2009). However, limited information exists in guidelines or standards that can be applied to long term observation programs of SPMC in marine and estuarine environments. Recent literature highlights only part of the uncertainties or problems related to the use of optical and acoustical sensors (Rai and Kumar, 2015; Rymszewicz et al., 2017). This study goes further as it reviews and discusses the benefits, limitations, and problems of present practices as they relate to the use of optical and acoustical sensors in long-term deployments, and formulates recommendations for acquiring the best quality-assured SPMC data possible. Other uncertainties or biases in the observation, which are for example related to the representativeness of the measuring location within the regional context, the interactions of the measuring infrastructure with the environment, or the disturbances by human activities are not part of this study. 


\section{Methods for long-term in situ SPMC measurements}

\subsection{Terminology}

SPM is a mixture of clay to sand-sized particles that can be detected in suspension and that consists of varying amounts of minerals from physico-chemical and biogenic origin, living and non-living organic matter, and particles from human origin, see Table 1 . The particles are considered to be in suspension as long as they do not form an interconnected matrix of bonds that prevents their mobility; this is the case when the concentration is below the gelling point (McAnally et al., 2007). The inherent properties of SPM (i.e. the concentration, size and composition) may change over time depending on the seafloor composition (cohesive and noncohesive sediments), the hydrodynamics, the measuring height above the bed and biological activity. Sand grains are generally limited to the near-bed layer (bed-load), while fine-grained sediments can be found throughout the water column. Charged particles such as clays and polymers may become attached to each other to form fragile structures known as flocs. The composition, size, density, structural complexity, and settling velocity of flocs vary as a function of turbulence, chemical environment (salinity) and bio-chemical composition (e.g. Eisma, 1986; Dyer and Manning, 1999; Droppo et al., 2005; Fettweis and Lee, 2017).

Long-term in situ measurements of SPMC in coastal seas and estuaries involve one or more optical and acoustical sensors and, as the ground truth reference, gravimetric measurements of filtered water samples. The combination of indirect and reference measurements requires two main calibration steps (sensor and model parameter calibration) at different moments during the workflow to extract reliable and homogeneous SPMC. These calibration steps are essential for relating changes in calibration constants (both sensor and model-parameter constants) to either sensor degradation or to natural variability in SPM inherent properties. Changes in SPM properties and concentration might be related to seasonal and geographical variations. The latter is typically occurring along the gradients from the estuary, coastal zone towards the offshore (Fettweis et al., 2006; Becker et al., 2013; Maerz et al., 2016; Many et al., 2016). Sensor calibration refers to the comparison of the output signal of an optical or acoustical sensor against laboratory standards (e.g. Thorne et al., 1991; Downing, 2006). For turbidity this is usually formazine or an alternative standard (AMCO clear $^{\circledR}$, StablCal ${ }^{\circledR}$ ), while for acoustical backscatter intensity a solution of standard spheres with a given size distribution, concentration and at a given distance from the transducer is used. Acoustic sensor calibration is not commonly applied for long-term measurements due to practical difficulties in setting up a laboratory device. Sensor calibration is necessary to convert the sensor output to various turbidity units (see below) or decibels ( $d B$ ). During long-term field operations the sensor 
calibration constants, relative to a standard may change. Any of these changes in the sensor calibration constants are inherent to the sensors and not to the environment.

Model parameter calibration refers to the regression between the sensor signal after sensor calibration and the corresponding real SPMC. The calibration should ideally be carried out in situ. This type of calibration is well distinguished from sensor calibration as it relies on natural particles and has to be carried out regularly, as well as each time the SPM inherent properties change significantly. If the sensors have been well-calibrated, then any changes in model calibration constants are caused by changes in SPM inherent properties.

Sample SPMC is the SPMC obtained from filtration and gravimetric measurements of water samples. As the real, in situ SPMC cannot be measured by any direct method we will use the sample SPMC as our reference, as it is less influenced by the inherent particle properties of the SPM (Neukermans et al., 2012a; Röttgers et al., 2014).

Sensor SPMC is a surrogate obtained by an acoustical or optical backscatter sensors or other type of sensors.

Turbidity refers to the optical water cloudiness caused by suspended particles and dissolved substances, which scatter and absorb light (Downing, 2005; Ziegler, 2003; Gray and Gartner, 2009). Turbidity does not have a SI unit, is not uniquely defined and depends strongly on the applied protocols. It is thus an arbitrary unit that is incomparable to measurements taken at other times and places or with different turbidity meters, which diminishes the comparability of turbidity data for scientific purposes (Downing, 2006). There are two international recognized methodologies for determining turbidity: the ISO Method 7027 (ISO, 1999) and the American EPA Method 180.1 (EPA, 1993). Both estimate turbidity, for the ISO method it is in formazine Nephelometric Units (FNU), and for the EPA method in Nephelometric Turbidity Units (NTU), respectively, and in both methods, the optical sensor to be used is a nephelometer that must measure side-scattered light at $90^{\circ}$. There are, however, some differences between the two methodologies. Turbidity following the EPA method, is poorly defined. The strengths of the ISO method include the use of a stable monochromatic near infrared light source of $860 \mathrm{~nm}$ with low absorbance interference with samples, which is critical in reducing the impact of particulate and coloured dissolved organic matter absorption and in having low amounts of stray light (Sadar, 1999). Sensors designed according to the ISO definition of turbidity provide thus a better basis for the comparability of measurements than those designed following the EPA specification (Barter and Deas, 2003, Nechad et al., 2009; Bright et al., 2018). The ISO compliant sensors are therefore recommended when no legal regulations require other protocols. As previously stated, both protocols refer to a formazine-equivalent turbidity value. If an alternative to formazine is used as the standard, it should be explicitly stated that turbidity 
refers to the alternative standard-equivalent NU or TU. The unit of the formazine (or alternative) standard is generally given in NTU, despite the fact that the standard solution can be created by following either the ISO or EPA protocol. In an ISO-compliant formazine solution of 1000 "NTU" an ISO-compliant side-scattering sensor will measure $1000 \mathrm{FNU}$, whereas an EPA-compliant side-scattering sensor will measure another value in NTU. The difference between FNU and NTU is mainly due to the spectral range of lights that are emitted by the instruments. For example, in waters with particles highly absorbing in the blue-green range, an EPA-compliant instrument measures lower turbidity values than an ISO-compliant instrument. Optical Backscatter Sensors (OBS) measure the particle backscattering of light at different angles of detection $\left(120^{\circ}-165^{\circ}\right.$, or even including side and forward scattering depending on the type of sensor); although highly correlated, the turbidity measured by an OBS and a Nephelometer is not necessarily the same. For example, Nechad et al. (2016) report a large variability in the relationship between in situ side- and back-scattering coefficients in the lower turbidity range ( $<20 \mathrm{FNU}$ ) and a convergence with increasing turbidity. Turbidity from backscatter devices should be expressed in Formazine Backscatter Units (FBU) for the near IR (830-890nm) light (ISO Method 7027) and Backscatter Turbidity Units (BTU) for the EPA Method 180.1 (Dogliotti et al., 2016; Nechad et. al, 2016). Often FTU (Formazine Turbidity Unit) or NTU are used as unit without specifying how the instrument measures the sample (side, back or forward scattering) or which protocol it follows (ISO or EPA). By adapting the correct units, the confusion on protocol or scattering angle can be avoided.

\subsection{Sensors used to obtain long-term SPMC time series}

\subsubsection{Sensitivity of optical sensors to inherent particle properties}

Various measurements of IOPs, which are defined as properties of the water column that are independent of the ambient light field, are used as proxies for SPMC. Optical sensors rely on the interaction of SPM with light at a given wavelength $\lambda$ through absorption $a(\lambda)$ and scattering $b(\lambda)$. For long-term measurements, the attenuation or the scattering at a given angle are mainly used to obtain the SPMC surrogates. Details on the optical properties of particles can be found e.g. in the Ocean Optics Web Book (2018). Available sensors can differ in wavelength and backscattering angle. The backscattering of light by particles, $b_{b}(\lambda)$, is theoretically calculated as the integration of the volume scattering function (VSF; denoted as $\beta$ $(\lambda, \theta)\left[\mathrm{m}^{-1} \mathrm{sr}^{-1}\right]$ with $\theta$ representing the scattering angle), over all backward directions. Boss and Pegau (2001) and Berthon et al. (2007) showed that $b_{b}$ is highly correlated to $\beta$ at angles between $120^{\circ}-140^{\circ}$, while Chami et al. (2006) found that the increase of $\beta$ at $\theta>150^{\circ}$ can significantly impact the backscattering. The backscattering of particles at large wavelengths $(\lambda>700 \mathrm{~nm})$ gives the best estimations for SPMC (Downing, 2006; Boss et al., 2009a). The effect 
of absorption by particles and coloured dissolved organic matter is higher at shorter wavelengths (Yentsch, 1962) which can impact (back)scattering or turbidity estimation by sensors that emit light at short wavelengths, as was highlighted by Sutherland et al. (2000) and Downing (2005). In more turbid waters, high amounts of particle absorption can influence the estimation of backscattering coefficient, even at longer wavelengths and should be properly corrected for (Doxaran et al., 2016).

As mentioned above, ISO Method 7027 (ISO, 1999) and EPA Method 180.1 (EPA, 1993) estimate turbidity $T$ in Formazine Nephelometric Units (FNU) or Nephelometric Turbidity units (NTU), respectively, such as $T=\frac{\beta\left(90^{\circ}\right)}{\beta^{F}\left(90^{\circ}\right)}$ where $\beta^{F}\left(90^{\circ}\right)\left[\mathrm{m}^{-1}\right]$ is the VSF at $90^{\circ}$ of a unit of formazine. As has been outlined above, other types of optical sensors such as backscatter sensors are widely used to measure turbidity and hence to estimate SPMC. In terms of sensor calibration based on successive dilution of a standard solution, this use of multiple sensor types does not cause problems as the sensor output will be compared, and then associated, to the standard solution that can be expressed in a turbidity unit. This calibration must then be used for quality control and to ensure the stability of the intrinsic sensor performance, and possible drifts induced by electronic failure or damages on optical windows that may induce maintenance and repair. The main issue comes when these instruments measure turbidity in situ or from natural water samples as IOPs (i.e. scattering efficiency $K$ ), in that case the VSF are dependent on particles shape (Slade et al., 2013), particles size and density, refractive index (Mishchenko et al., 2002; Boss et al., 2004), and colour (Sutherland et al., 2000; Hatcher et al., 2000; Bright et al., 2018). Generally, $b(\lambda)=K(\lambda, r) N(r) \pi r^{2}$, where $K$ is the scattering efficiency factor for non-absorbing particles, $N$ the number of particles, and $r$ the particle radius. For spherical particles $S P M C=\frac{4}{3}$ $N \rho \pi r^{3}$, where $\rho$ is the dry density (i.e. particle mass divided by the particle volume), resulting in a ratio of turbidity to SPMC that is inversely proportional to the particle radius and density (Sutherland et al., 2000; Babin et al., 2003):

$$
b(\lambda)=\frac{3}{2} K(\lambda, r) \frac{S P M C}{\rho r}
$$

The SPM dry density (proportional to the excess density) can, depending on the particle composition, be anywhere in between the range of well below $100 \mathrm{~kg} \mathrm{~m}^{-3}$ for the organic flocs and up to $2650 \mathrm{~kg} \mathrm{~m}^{-3}$ or more for the individual mineral particles. In case the SPM consists of flocs the variations in density and size can be very large, with the densest flocs having the smallest size and vice versa. The consequence of this is that the dependence of $b(\lambda)$ on $\rho r$ is weaker than its dependence on $1 / r$ (Bowers et al., 2009) but can still be significant (Gibbs, 1985; Babin et al., 2003; Baker and Lavalle, 1984). This demonstrates that a change in the composition of particles and/or their shape, size and density affects the turbidity measured by 
a side- and backscatter sensor (e.g. Binding et al., 2005; Neukermans et al., 20012b; Zhang et al., 2014; Druine et al., 2018), but also indicates that the model parameter calibration of optical backscatter instruments using a single optical property (backscattering coefficient or beam attenuation) against sample SPMC is often successful over a wide range of particle sizes (Boss et al., 2009b; Bowers et al., 2017) as long as SPM characteristics do not change significantly over the observed period.

\subsubsection{Sensitivity of acoustic sensors to particle properties}

Some acoustics devices commonly used in coastal areas, such as ADVs or ADCPs are primarily designed for current velocity measurement, while others (ABS) are specifically designed for measuring SPMC at a single point (Guerrero and Di Federico, 2018) or in a vertical profile (Hay and Sheng, 1992). Analogously to optical devices, the emitted acoustic wave, at a given frequency, interacts with particles in suspension while propagating in the medium and are backscattered to the receivers (Thorne and Hanes, 2002). The recorded volume backscattering strength $\left(S_{v}\right.$, in $\left.\mathrm{dB}\right)$ is a proxy of the SPM concentration, but is also strongly modulated by SPM features such as size, density and shape, depending on the acoustic wavelength. Originally used in sandy environments (Sheng and Hay, 1988; Thorne and Hanes, 2002), these devices are now routinely deployed in fine sediment environments (e.g. Gartner, 2004; Hoitink and Hoekstra, 2005; Merkelbach, 2006; Merkelbach and Ridderinkhof, 2006; Tessier et al., 2008; Sahin et al., 2013). The sonar equation (Urick, 1975) is commonly used to relate $S_{v}$ (in $\mathrm{dB}$ ) and SPMC concentration, including acoustic signal correction for geometry compensation, spherical spreading, and water and particle attenuation:

$$
\frac{S}{N}=S L-20 \log _{10}\left(\psi R^{2}\right)-2 \int_{0}^{R}\left(\alpha_{w}(r)+\alpha_{s}(r)\right) d r+S_{v}+10 \log _{10}\left(\varphi R^{2} \frac{W S}{2}\right)
$$

and

$$
S_{v}=10 \log _{10}\left(\frac{S P M C \dot{\sigma}}{\rho_{s} v_{s}}\right)
$$

where $S / N$ is the signal to noise ratio $(\mathrm{dB})$ received by the device. After Gostiaux and Van Haren (2010), Mullison (2017) specifies the values of $S / N$ in function of the raw echo readings (in counts) of the ADCP devices. $S L$ is the source level (dB); $R$ is the along-beam distance from transducers, $a_{w}$ and $a_{s}$ respectively the water and sediment attenuation, $\varphi$ the angular aperture; WS the cell size and $\psi$ the near field correction. $\bar{\sigma}_{,} \rho_{s}$ and $v_{s}$ are particle features: backscattering cross section, particle dry density and volume respectively. Comparably to optical devices, the relationship between the acoustic backscatter and SPMC strongly depends on the nature, size, density and shape of the particles, both for the estimation of the particle attenuation and for the calculation of the backscattering cross section (Fugate and Friedrichs, 2002; Ha et al., 2009; Salehi and Strom, 2011; Rouhnia et al., 2014), achieved 
using theoretical acoustic models (Stanton, 1998; Thorne and Hanes, 2002; Thorne et al., 2014). However, contrary to optical devices, the sensor calibration is not routinely (often never) operated due to the difficulty to access requested laboratory facilities. Hence this step is often skipped and quality check is based on comparison with other in situ sensors or SPMC samples.

\subsubsection{Conversion of acoustical and optical sensor output to SPMC}

The relationship between OBS or nephelometer signal and SPMC is almost linear as long as the sensor is not deployed in highly concentrated waters (Downing, 2006), and the simplest model is a linear regression model. The same holds for single point acoustical sensors (ADV) or for the first bin of a profiling acoustical sensor, where the target volume is very close to the sensors. As far as SPMC are lower than several $\mathrm{g} / \mathrm{l}$, a direct empirical relationship can be built such as $\log _{10}(S P M C) \sim S_{v}$, where $S_{v}$ can be related to the signal/noise ratio (Fugate and Friederichs, 2002; Voulgaris and Meyers, 2004; Verney et al., 2007; Ha et al., 2009; Salehi and Strom, 2011). For profiling acoustic sensors, the sonar equation should be considered to correct for the signal loss along the acoustic path. The conversion factor from counts to $\mathrm{dB}$, as commonly used in acoustics, is typically provided by the manufacturer. Close to the transducer, the acoustic signal has to be corrected for near-field effects (Downing et al., 1994) and for ringing effects that may affect the first bins, in particular when blank distance is set too small in the configuration parameters. Corresponding data cannot be corrected and should be discarded (Muste et al., 2006). A formulation for the water absorption coefficient was proposed by e.g. François and Garrison (1982a, 1982b) and later simplified by Ainslie and McColm (1998), who showed that their result did not differ from the original equation more than the accuracy error. The sonar equation yields the so-called water-corrected backscatter, which is a property of the suspension at all locations along the acoustic path. Subsequent processing depends on the SPMC. In case of moderately turbid environment, i.e. lower than $100 \mathrm{mg} / \mathrm{I}$, sound attenuation by SPM is usually neglected as it is one or two orders of magnitude lower than the water absorption coefficient (Ha et al., 2011). SPMC is then either determined by applying an appropriate calibration, similar to single point optical sensors, or by a theoretical acoustic model. In the latter case, physical properties of the transducer and of the SPM must be exactly known, which are rarely available. If SPMC exceeds $100 \mathrm{mg} / \mathrm{l}$, sediment absorption should be considered. However, this term is a function of the SPM concentration, which is also the unknown of the calculation. The inversion problem is solved by iterative methods (Thorne et al., 1994; Holdaway et al., 1999). This technique is efficient but requires assumption or knowledge about SPM characteristics (size, density) and is based on the choice of an acoustic model adapted to the observed SPM, and may in some specific case exponentially propagate uncertainties and fail to estimate SPMC (Becker et al., 2013). 
Version: August 11, 2019;

Theoretical acoustic models were originally built to simulate the physical interactions between particles and the acoustic signal (Sheng and Hay, 1988, Medwin and Clay, 1998) and were applied to sand particles in suspensions (Thorne and Hanes, 2002). These models were later adapted to represent low density aggregates of SPM (Stanton, 1998; McDonald et al., 2013, Thorne et al., 2014). Differences between models mainly appear in the methodology to calculate the total scattering and backscattering cross section as well as the compressibility of flocs and their ability to interact with sound. Merckelbach and Ridderinkhoff (2006) and Nauw et al (2014) observed that at strong currents (>1 m/s) acoustical backscatter exceeds the linear relationship to sample SPMC noted at lower currents. This was not due to changes in the particle-sizes and the non-linearity was compensated for, based on a model that suggests a transition from random phase to coherent particle backscatter by turbulence-induced variability in in the spatial distribution of SPMC (Merckelbach, 2006). More research is however required to understand the cause of this effect.

\subsection{Existing international guidelines and standards}

In the European framework guidelines for SPMC determination consider only the collection of water samples. OSPAR's Joint Assessment and Monitoring Programme JAMP (2012) refers to the ICES TIMES report by Yeats and Brügmann (1990). The Trilateral Monitoring and Assessment Program for the Wadden Sea (TMAP) refers in their handbook on nutrients (TMAP, 2009) explicitly to the corresponding guidelines of JAMP. HELCOM (2015) treats SPMC as a cofactor in water analysis and keeps its determination by filtration according to ISO (1997). In a global framework, the IOC-EU-BSH-NOAA-(WDC-A) International Workshop on Oceanographic Biological and Chemical Data Management did not include SPMC in the list of bio-geochemical bulk parameters (IOC, 1996). For the ARGO programme, Quality Control (QC) manuals for BioArgo particle backscattering measurements are yet in development (Schmechtig et al., 2015), the main purpose of these measurements is the use of the backscattering coefficient as proxy for POC concentration (IOCCG, 2011). The National Oceanic and Atmospheric Administration NOAA does not provide standards for SPMC measurements in official documents. In some technical reports, however, the methods to obtain SPMC are described, always using gravimetric analyses of water samples (e.g. Pait et al., 2015). For the large-scale integrated ocean and coastal ocean observatories, such as IOOS in the United States (www.ioos.us) and IMOS in Australia (www.imos.org.au), no guidelines for best practice of SPMC measurements are put forward. The U.S. Geological Survey provides a number of documents comprising guidelines for the sampling (Edwards and Glysson, 1999), for the lab analysis of the samples (Matthes et al., 1992; Shreve and Downs, 2005), for the proper layout and operation of field stations and sensors to meet accuracy and precision requirements (Wagner et al., 2006). They 
Version: August 11, 2019;

also describe how to compute time series of SPMC and their related loads using proper statistical regression models (Rasmussen et al., 2009, Topping and Wright, 2016). These guidelines were developed for measurements in rivers, but many of them can be transferred to estuaries and coastal seas in a straightforward way, except that estuarine and coastal SPM exhibits much higher variability on texture and composition. 
Version: August 11, 2019;

\section{Sources of uncertainties}

Uncertainties, as described in Ramsey and Ellison (2007) and ISO (2008; 2017), hamper data quality and may arise from mal-functioning of sensors (3.1), the environment influencing the sensors without changes in SPMC (3.2), SPMC sample collection and analysis (3.3), modelling of sensor output to sample SPMC (3.4), and additional uncertainties arising from human error and uncontrolled environmental boundary conditions (3.5).

\subsection{Sensor}

Sensor related uncertainties occur if the sensor output changes over time unrelated to changes in inherent particle properties or SPMC. Concerning optical backscatter sensors this is caused by variations in voltage supply, changes in the transmittance of the window that is the interface between the sensor and the water or other degradations. Concerning acoustical backscatter sensors, the main sensor related uncertainty is related to battery power. The reason that no other sensor related uncertainties are documented is due to the limited availability of facilities to test acoustic sensors in a lab, and that in contrast to optical sensor, no standards and norms exists so far to test and calibrate acoustical backscatter sensors. Other uncertainties may arise from the drift of the internal clock of the sensor or the data logger in particular in case of long term monitoring.

\subsubsection{Drift with time}

Optical sensors are subject to gradual decreasing transmittance of the sensor window during deployment due to scratches caused by the collision of particles. Therefore, the factory calibration parameters should be used with care and be checked regularly. The damage of the transmission and detection windows is often not visible by visual inspection. A re-calibration of 11 Seapoint turbidity sensors operated by HZG in the laboratory for example revealed that the average slope between factory calibration and formazine recalibration agreed within $1 \%$, but the individual slopes showed discrepancies up to $\pm 30 \%$ with a standard deviation of $15 \%$. Figure 1 shows a comparison between an OBS3+ factory calibration and a sensor calibration obtained after 329 and 421 days highly turbid (SPMC > 1g/l) sea water. Using the factory calibration constants without recalibration would indicate an apparent increase of the turbidity. Any of these changes in the calibration constants are inherent to the sensors itself and the need of recalibration has to be specified by the user based on the required precision.

Variations in voltage supply result in a drift, which is unrelated to variations in SPMC or inherent particle properties. In clear water the output of for example an OBS3+ will be a minimum voltage, increasing to a maximum voltage at the maximum turbidity range. The sensor needs to be fed by a constant minimum voltage in order to give good data. Lower voltage supply results in an apparent decrease in turbidity. Figure 2 a shows an OBS3+ in low (0- 
Version: August 11, 2019;

$2000 \mathrm{BU})$ and high (0-4000 BU) range that has been fed with different input voltages while measuring in a standard of 2000 AMCO Clear ${ }^{\circledR}$-equivalent BU. For an input voltage of more than $6 \mathrm{~V}$ the low range registers a constant value of $5.1 \mathrm{~V}$ (saturation), while the high range registers about half of it $(2.5 \mathrm{~V})$. If the supplied voltage decreases, the signal registered by the OBS3+ decreases as well. An example of a time series collected with an OBS that received a low battery voltage and another one with a normal voltage supply is illustrated in Figure $2 \mathrm{~b}$. The recorded SPMC signal of the first sensor was on average about $90 \%$ lower than it should be. After sensor calibration with lower voltage supply the data could be corrected. Battery power affects also acoustical backscatter sensors (Tessier et al., 2008). From the registered battery voltage during the deployment the emitted power can be converted into the correction factor $P_{d b w}$ calculated as $P_{d b w}=10 \log \left(P^{2}\right) / R$, with $\mathrm{R}$ the electrical resistance. If only one of the transducers of profiling acoustical sensors supplies more or less power than the others, then this can be corrected using the other transducers (Tessier, 2006).

\subsubsection{Sensitivity}

Some optical backscatter sensors, such as an OBS3+, are dual range sensors having both a lowand a high-range output. The two measuring ranges increase the resolution of the measurements, but each output is more accurate when its designed turbidity range matches its calibrated range. The RMS error between high and low range output depends on the turbidity. For example, for an averaged turbidity around 20 the low range $(500 \mathrm{BU})$ recordings during a tidal cycles differed by about $2 \%$ with the high range ones (2000 BU). When increasing the ranges (low: $1000 \mathrm{BU}$, high $4000 \mathrm{BU}$ ) then the relative difference increased to $24 \%$. The optimal range is to be chosen according to the expected range of turbidity values in the field.

\subsubsection{Inter-sensor variabilities}

The aim of multi-sensor calibration is to generate a reliable model parameter calibration to estimate SPMC at long-term monitoring stations by repeated field surveys and to record variabilities caused by the use of similar types of sensors. Figure 3 shows the result of a simultaneous calibration of three OBS3+ sensors during a tidal cycle. The RMSE between the three OBS3+ sensors, which have been calibrated against the same sample SPMC, was $2.6 \%$ for $1 \mathrm{~Hz}$ sampling and $2 \%$ for an averaging over $60 \mathrm{~s}$. The difference between sensor outputs is changing during the course of the measurements. This points to small-scale variations in the SPMC (sensors are located about $50 \mathrm{~cm}$ from each other) and to different sensitivities of the individual sensors. These examples presented strengthen the necessity of sensor calibration (difficult to achieve for profiling acoustic sensors) and model calibration for each sensor separately.

\subsection{Environmental related uncertainties}


Environmental related uncertainties occur when the sensor output changes over time unrelated to changes in inherent particle properties or SPMC. This occurs when the signal is for example attenuated by biofouling or the occurrence of air bubbles. Another environmental uncertainty is specific to optical sensors and occurs when the emitted light beam is strongly attenuated and backscattered light decreases with increasing sediment concentration.

\subsubsection{Biofouling}

Biofouling or other type of fouling can limit the accuracy and quality of long-term SPMC measurements within a week, depending on the season and the environment. Biofouling occurs in four phases starting with the development of an organic film, followed by primary and secondary micro colonizers and finally tertiary colonizers attach to the micro-fouling film (Abarzua and Jakubowski, 1995). The impact of these different phases on the recorded signal remains difficult to estimate and can result in a temporary or permanent increase of the backscattered signal due to additional reflection or a decrease due to attenuation (Kerr et al., 1998; Delauney et al., 2010). Increase of the backscattered signal can also be caused by plant or artificial (e.g. fishnet) filaments trapped by the measuring infrastructure and constantly or ephemerally influencing the detection volume. There are several methods and reasons to protect the sensors against biofouling (Ridd and Larcombe, 1994; Manov et al., 2004; Whelan and Regan, 2006). The most obvious reason is to obtain good quality data. Another one is that the fouling development on the whole measuring infrastructure can disturb the properties of the study site. For example, the growth of epi-fauna on the infrastructure may influence the measurements as they trap, accumulate and temporarily release SPM (Baeye and Fettweis, 2015). Effectively biofouling results in a gradual and continuous drift of the signal over time together with an apparent decrease in sensitivity (Dolphin et al., 2000; Downing, 2006). Permanent biofouling events will gradually change the backscatter intensity and can only be corrected if a non-affected reference sensor is at hand. The reference sensor can be single beam acoustic or optical backscatter sensor or a not-affected beam of an acoustic profiler. The onset of a change in the SPMC signal due to biofouling is difficult to identify and therefore should be based on the hypothesis that the observed changes cannot any more be explained by known physical behaviour of SPMC variations.

Figure 4 is an example of epifauna (barnacles) growing on an OBS that resulted in an increase of backscatter intensity until saturation and thus an overestimation of the SPMC. The timing of biofouling was estimated at the point where the ratio of the affected sensor to a non-affected one started to increase monotonically. Biofouling has also been observed on moored up-ward looking ADCPs. A straightforward identification of these events is possible by computing the cross-beam backscatter ratio. Expressed in $\mathrm{dB}$, this ratio is computed as the difference between 
the maximum and the minimum of all backscatter intensities recorded at a given depth by all beams of the sensor. In case of an ADCP, each ratio value is then computed over a typical ensemble composed by three or four data points (Jourdin et al., 2014, their eq.10). Hence, in a homogeneous medium and in absence of biofouling, measured values of the cross-beam backscatter ratio are close to the sensor noise (typically $1 \mathrm{~dB}$ ). But a drop of up to $10 \mathrm{~dB}$ has been observed in ADCP moorings deployed off the French coast (Jourdin et al., 2014), while in an ADCP mooring off the Belgian coast a gradual drop of more than $20 \mathrm{~dB}$ in acoustic backscatter intensity have been recorded. A value of $10 \mathrm{~dB}$ leads to under-estimation of the suspended sediment concentration by one order of magnitude, over the entire acoustic profile. If only one beam of an ADCP is affected by biofouling, this can be corrected by applying the median function to all beams as explained in Jourdin et al. (2014). Figure 5 provides an example of permanent biofouling resulting in a gradually decrease of the acoustic backscatter intensity during the mooring, while in Figure 6 episodic biofouling events are shown. In any case, acoustic and optical instrument should be serviced at least once a month and at higher frequency during periods of strong seasonal bloom (van der Hout et al., 2017).

\subsubsection{Saturation and ambiguity problems}

SPMC in the field may vary from several $\mathrm{mg} / \mathrm{l}$ to a few $\mathrm{g} / \mathrm{l}$. At these ranges, the OBS output increases with SPMC, and the gain setting is adjusted by the manufacturer for an optimized sensitivity at the expected concentrations in the field. However, for too high SPMC values, saturation occurs and the OBS output will show a plateau as illustrated in Figure 4 (the OBS at 0.2 mab from day 137 onward). The individual setting of certain probes can be adjusted (see section 3.1.2) to broaden the range of SPMC before saturation.

For many OBS probes, beyond the saturation range, the output signal decreases with increasing concentration as was firstly observed in the field by Kineke and Stenberg (1992). They attributed this trend to the partial blockage of the emitted beam by highly concentrated suspensions, the reduction of the scattering volume relative to the area of detection. Figure 7 shows typical bell-shaped backscatters curve, meaning that a given OBS output can correspond to two possible SPMC values. The two values that can be obtained (i.e. the up and over response) depend on the sensitivity of the individual sensor. This bell-shaped response can be particularly useful when measuring near-bed very high concentrations (Sottolichio et al., 2011). However, because of this ambiguity, the determination of SPMC in highly concentrated waters (>4 $\mathrm{g} / \mathrm{l}$ ) with a single-point measurement is not always possible. A correction is possible in case of vertical profiles, because SPMC is continuously increasing downward and the decrease of the near-bed OBS output can be readily related with an increase of SPMC (Kineke and Sternberg, 1992). However, this does not help in case of time series, obtained by one probe at fixed height 
above the bed. The use of a second probe above the other could help to solve the ambiguity of one of the two probes (Figure 7). Acoustic backscattering sensors show the same type of response than optical probes, and the ambiguity issue applies thus also to acoustic devices, such as single point ADV sensors (Ha et al., 2009; Sottolichio et al., 2011).

\subsubsection{Air/gas bubbles}

Due to the high acoustic impedance or the very different refractive indices between gas and water, the volumetric backscattering strength measured by acoustical sensors or the scattering efficiency measured by an optical sensor is sensitive to the presence of air or gas microbubbles (1 to $500 \mu \mathrm{m}$ in radius) in water. These bubbles are mainly generated at the sea surface by wave breaking or white-capping (e.g. Schwendeman and Thomson, 2015) and as a consequence the subsurface scattering is perturbed by the sea state (e.g. Klein, 2003; Downing, 2006; Puleo et al., 2006) from surface up to depth (in $\mathrm{m}$ ) that approximately equals the wind speed (in $\mathrm{m} / \mathrm{s}$ ) squared divided by 15 (Wang et al., 2016) or in the vicinity of bubble plumes (Nauw et al., 2015). Indeed, the finest bubble population of typical size less than $50 \mu \mathrm{m}$ in radius penetrates within the water column where it can remain in near-equilibrium suspension (e.g. Randolph et al., 2014). Outside the surf zone, bubbles can be carried down in form of plumes or patches by convergent fronts or wind-induced down-welling to depths up to $30 \mathrm{~m}$. The vertical distribution of wave bubbles decays exponentially with depth resulting in a similar decrease of the backscattering strength at lengths scales of 0.5 to $5 \mathrm{~m}$ (Wang et al., 2016). Expressed in decibels the backscattering intensity displays a linear decrease with depth (e.g. Wang et al., 2011) and this property together with measurements of the wind speed $\left(U_{10}\right)$ and wave height in parallel can be used to discard parts of profiles affected by wave bubbles, see the example in Jourdin et al. (2014).

\subsubsection{Density stratification}

As reminded in Eq. 2, acoustic backscatter inversion requires first correction for water attenuation, which is a function of the acoustic frequency, temperature, salinity and depth (Lurton, 2010). In estuaries and coastal seas, strong density stratification can be observed, and may introduce noise in the backscatter intensity inversion if not corrected properly: for a 1200 $\mathrm{kHz} \mathrm{ADCP}$, the water attenuation coefficient can vary from $0.2 \mathrm{~dB} / \mathrm{m}$ to $0.55 \mathrm{~dB} / \mathrm{m}$. For a given average temperature, the water attenuation coefficient variability is around $0.1 \mathrm{~dB} / \mathrm{m}$ for a $1200 \mathrm{kHz}$. The uncertainty introduced by stratification was examined using ADCP and CTD measurements in the Seine estuary, where strong temporal and vertical stratification are observed as shown in Figure 8 (Sahin et al., 2017). In the given example, not considering water salinity/temperature variability may induce uncertainty on absolute acoustic backscatter intensity of the order of $1 \mathrm{~dB}$, which results in a systematic error of about $\pm 40 \%$. Similar 
uncertainties can be expected offshore, as salinity/temperature variability is lower but depths are larger.

\subsection{Sample related uncertainties}

Sample related uncertainties occur during collection and analysis of the sample SPMC, i.e. the filtering and weighing. A diversity of methods and equipment are used to collect water samples, such as Niskin bottles, Go-Flow bottles and stand-alone or on-board sea water pump or suction systems. The bottles attached to a profiling frame, or the intake for the pump system should be close enough to the detection volume of the sensors, which are deployed for model calibration. Whereas the occurrence of SPM in plumes creates random scatter in the relation between filter weights and sensor outputs, the occurrence of permanent gradients causes systematic errors. Therefore, one should take beforehand knowledge of the spatial gradients to define "close enough". It is not possible to present a general number here, as the gradients are dependent on the spatial extension of the studied basins or estuaries or the typically prevailing hydrodynamic energy inputs. In most cases samplers are mounted on the same frames as the sensor packages. Scales of horizontal gradients are normally in the order of more than $10 \mathrm{~m}$ and therefore less critical than vertical that are typically in the order of a meter or less.

Standard protocols for dry mass SPMC determination (Strickland and Parsons, 1968; Pearlman et al. 1995) and additional procedures to partition SPMC into its organic and inorganic content (e.g. loss on ignition, element analysis) are straightforward: They include filtering a specific volume through a pre-weighed filters, washing of sample filters and filter edges with deionized water (or MilliQ water) to remove salt, drying, and weighing of the filter to determine the dry mass of the SPMC. In early years, paper filters were used; later membrane filters (Banse et al., 1963) have been in use, while nowadays often glass-fibre filters (type GF/F or GF/C) are used. The removal of the salt in filters is a critical source of uncertainty, which if not well done, will lead to an overestimation of the SPMC (Neukermans et al., 2012a). Additional uncertainties are related to the determination of the dry mass and filter volume and the amount of structural water in minerals (Barillé-Boyer et al., 2003). Most uncertainties are related to a constant mass offset (salt, weight and volumetric determination) and are, hence, relatively small when enough mass of particulates (several milligram) is collected on the filter. The collection of a sufficient mass is easy to achieve in turbid nearshore or estuarine waters dominated by mineral material, but more difficult in low turbid organic-rich waters or for example during a Phaeocystis bloom. In the latter case the gelatinous algal colonies may clog the filter before enough material is collected and prevent the removal of the salt. In cases were the sample mass on the filter is not very high, errors due to salt are significant (Banse et al., 1963; Stavn et al., 2009; Röttgers et al., 2014). In cases were the sample is turbid (>100 mg/l), the main uncertainty is often caused by 
the difficulty to homogenize the sample prior to subsampling and filtration (Fettweis 2008). Methodological improvements include the optimization of the filtration volume and cleaning of the filter margin after the funnel is removed (Neukermans et al., 2012a); the correction of the salt mass bias using procedural control filters (Stavn et al., 2009), the filling of the filter margin with salt-free water before filtration and the determination of a SPMC free of the salt-bias using a set of different sample volumes (Röttgers et al., 2014).

The uncertainty in sample SPMC decreases with increasing SPMC as shown for a data set of about 2500 sample SPMC from the Belgian nearshore obtained with the method of triplicates with the same volume using GF/C filters. The uncertainty (expressed as the RMSE of the triplicates divided by the mean value) is highest (8.5\%) for sample SPMC lower than $5 \mathrm{mg} / \mathrm{l}$ and decreases with increasing SPMC to $6.7 \%$ (<10 mg/), 3.5\% (10-50 mg/l) and 2.1\% (>100 mg/l). This error corresponds to the uncertainty introduced if only one replica was used instead of three replicas. In case of triplicates the error is limited by excluding sample SPMC with a RMSE exceeding a threshold value. The need of subsamples with different volumes versus subsamples with the same volume has been checked from samples collected in three stations with different turbidity in the southern North Sea. The estimated slope, i.e. the SPMC, has an uncertainty that can be higher than $1 \mathrm{mg} / \mathrm{l}$ (>30\%) for clearer waters (SPMC about $3 \mathrm{mg} / \mathrm{l}$ ) and then it drops to about $0.3 \mathrm{mg} / \mathrm{I}$ for SPMC of about $11 \mathrm{mg} / \mathrm{l}$ (3\%) or $25 \mathrm{mg} / \mathrm{l}$ (1\%). Using the method with the same volume, the uncertainty is about $0.3 \mathrm{mg} / \mathrm{l}(10 \%)$ for the clearest sample, about $0.4 \mathrm{mg} / \mathrm{l}$ (4\%) for SPMC around $11 \mathrm{mg} / \mathrm{l}$ and about $0.8 \mathrm{mg} / \mathrm{l}$ (3\%) for SPMC of around $25 \mathrm{mg} / \mathrm{l}$. The relative difference between the estimated SPMC using this method and the different volume method (applied three times, and averaged) is about $11 \%$ for clear waters, $6 \%$ and $4 \%$ for higher levels of turbidity. One should consider that the uncertainty (standard deviation) of the SPMC through averaging of triplicates with the same volume is not the same uncertainty as that obtained via the slope determination using the different volume method. The latter includes a random and a systematic error due to the offset by salt, while the same volume method provides only a measurement error. The systematic error is not detected in the same volume method, which explains the apparent lower uncertainty of this method, especially at low SPMC as compared to the different volume method. The method with different volumes is more accurate, the latter holds especially in low SPMC waters, where the effect of salt is important (Röttgers et al., 2014).

\subsection{Relating sensor output to sample SPMC}

Modelling techniques establish a statistical relationship between turbidity or acoustical backscatter intensities and reference SPMC data, i.e. the sample SPMC (ISO, 2014). This relationship is applied to convert the time-series of turbidity or acoustical backscattered signal 
into SPMC. However, for acoustic devices such as an ADCP a calibration against in situ water samples is challenging, as samples are often difficult to collect next to the profiling sensor and as the property of the SPM may change over the water column. For these cases the SPMC derived from optical sensors measuring close to the ADCP is often used as reference SPMC. It must be noted that the sample SPMC or the optical-derived SPMC is collected at a given location in a confined volume, while the acoustic backscatter signal from a profiling sensor is the mean of a user-defined volume, which is generally much larger than the one of the sample SPMC. This procedure will add additional uncertainty to the model.

The first two sections deal with a purely technical aspect, i.e. the biases involved with the choice of the linear regression model. The third section addresses uncertainties that are caused by systematic changes of the particle IOPs or IAPs that will result in varying proportional factors between the detected signal and SPMC that are not detected during the generally short calibration periods. A general bias in the model parameter calibration relation may be caused by the spatiotemporal mismatch between the sample and the sensor, by local changes in the turbulence and floc size caused by the sampling device or by the calibration period that is not necessarily representative for the whole unsupervised time series. The fourth section deals with the inconsistencies between parallel optical and acoustical data that are caused by the uncorrelated and random variations in the IOPs and IAPs.

\subsubsection{Modelling techniques}

The relationship between optical sensor output to SPMC or the acoustic backscatter to the $10 \log _{10}$ (SPMC) can be modelled using a linear model (see section 2.2). The model can be applied to the linear domain or the log-log transformed domain (the latter only for turbidity data). Mostly, after quality checks of the data, linear regression finds optimal regression parameters by minimization of the squared differences between the values of the dependent variable and the regression line ( $X^{2}$ method, further called LSQ). In general, however, different options for the linear regression method can be considered that lead to different values in the regression parameters for the same data set and different levels of predictability of SPMC from optical turbidity or acoustic backscatter intensity.

The first option is the choice of the independent (explanatory) and dependent (response) variable. As one finally wants SPMC as a function of turbidity, mostly SPMC is used as dependent variable. On the other hand, as turbidity depends on SPMC and not vice versa, this requires turbidity as dependent variable and then compute SPMC from turbidity using the inverse of the calibration regression. As another option, one may regard both SPMC and turbidity not strictly causal dependent on each other. This situation may be adequate for cases where water sampling locations and turbidity detections volumes differ by typically more than 
the coherence lengths of the SPMC in the observation area. Then the minimization of the difference between data and regression line should take place perpendicularly to the regression line solving an Eigenvalue problem. This method leads to symmetric results no matter whether SPMC or turbidity is the dependent or independent variable.

In most real data sets, the assumptions for linear regressions are not met (e.g. Gilbert, 1987) and ordinary least-squares fitting can have undesired sensitivities both in best model parameter or confidence interval estimations. In this study, two examples of robust fitting are contrasted with ordinary least-squares, i.e. the Theil-Sen estimator and the "iteratively reweighted least squares" (further called Robust) method (Press et al., 1989).

To overcome the problems with data heteroscedasticity, $\log _{10}$ - transformations of the variables can be applied, a decision that should be based on the examination of the model residuals (Helsel and Hirsch, 2002). This transformation has also the advantage that the distribution of data along the independent variable is more homogeneous as there is very often a bias in the coastal water sample data sets towards lower SPMC. The re-transformation of the regression line into the original units introduces a bias that arises as the regression estimates are computed from means in log units unequal to the mean in original units. Helsel and Hirsch (2002) recommend the usage of the non-parametric "smearing" estimator

$$
B C F=\frac{\sum_{i=1}^{n} 10^{e_{i}}}{n}
$$

introduced by Duan (1983) as bias correction factor (BCF) with $n$ the number of samples, and $e_{i}$ the residual of sample $i$ in log-units. This BCF is not symmetric when using the Eigenvalue regression in the log space as SPMC and turbidity have different units.

Figure 9 shows the calibration of an OBS against sample SPMC for the different methods and the exchange of dependent and independent variables. It also shows that outside the data range available for calibration, the uncertainty may increase significantly. The next example the direct calibration of an ADCP (1 MHz Nortek AWAC) against sample SPMC in a moderately turbid environment $(<100 \mathrm{mg} / \mathrm{l}$ ) is shown (Figure 10). The direct calibration with sample SPMC was performed for the surface and bottom samples separately as they originate from different population of particles in suspension, i.e. surface plume and bottom resuspension. The data are from a coastal observatory located at the mouth of the Seine estuary and consists of a bottom station equipped with an ADCP and a Wetlabs NTUSB optical backscatter sensor (Wetlabs OBS) and a surface buoy measuring turbidity with a similar Wetlabs NTUSB sensor.

In Figure 11 the model calibration of two acoustic sensors ( $3 \mathrm{MHz}$ Sontek ADP with series number M284 and M947) is shown using the OBS-derived SPMC as the reference SPMC. The data is from a one-year time series in 2013 collected in the turbid Belgian nearshore area at 
about $2 \mathrm{~m}$ above the bed (Fettweis et al., 2016). Both acoustic sensors have measured about half of the period at a burst rate of 15 minutes and all the available OBS and ADP data pairs have been used for the model calibration (M284: 12280; M947: 14923). The OBS sensors have been calibrated twice during the period against sample SPMC. The model calibration (M284: $\left.R^{2}=0.4 ; M 947: R^{2}=0.6\right)$ shows strong scatter in the data and thus a high uncertainty in the acoustic derived $10 \log _{10}$ (SPMC) versus acoustical backscatter in $\mathrm{dB}$. This is indicated by the dashed lines in Figure 11 representing the results of 7 different regression models. Their slopes differ by a factor of 1.8 and the associated uncertainties by even a factor of 3 within the data range when the OBS-SPMC data are re-transformed to SPMC. Other studies report similar (up to 0.8 ) ranges of $R^{2}$ (Fugate and Friedrichs, 2002; Gartner 2004; Dufois et al., 2014). The difference between OBS and ADCP estimates of SPMC during a one-week deployment in San Francisco Bay was $8 \%$ to $10 \%$ relative to OBS range and about $35 \%$ to $40 \%$ relative to OBS mean (Gartner, 2004).

\subsubsection{The choice of the linear regression model}

To estimate the uncertainties introduced by the choice of a specific regression method, we applied the different regression methods as described in section 3.4.1 to eleven data sets with $\mathrm{R}^{2}$ between 0.58 and 0.99 . With three minimisation schemes (LSQ, Eigenvalue and Robust fit) plus the Theil-Sen estimator, exchanging turbidity and SPMC as independent variable and taking the data with and without $\log _{10}$-transformations we obtain 15 combinations per data set, as for the non-transformed data the eigenvalue method is symmetric to the choice of abscissa and ordinate. The data sets consist of pairs of sample SPMC and turbidity collected at various location with Hach nephelometers (North Sea, English Channel, Mediterranean Sea, French Guiana coast, Gironde estuary, Rio de la Plata) and Seapoint sensors (Wadden Sea, North Sea, Oosterschelde estuary, Weser estuary). As an example for a field data set $\left(R^{2}=0.61\right.$, 26 samples), Figure 9 depicts the 15 regressions. Within the range of the calibration data, the different model results differ by some $20 \%$, but they increase significantly beyond the data sample range. In the following, we define Regression Spread (RS) as the percentage difference between SPMC computed from the regression models with highest and lowest slope as a function of turbidity. In Figure 12a the RS derived from the seven regression models for the non-transformed (i.e. linear scale) sample-SPMC/turbidity data pairs versus data set $\mathrm{R}^{2}$. Figure 9 exemplifies that within each data set RS depends on the value of the turbidity relating to the mean turbidity. To make the regressions of all data sets comparable, RS was computed for different z-transformed (standardized) turbidities, $T_{Z-s t d}$ defined as

$$
T_{Z-s t d}=(T-\grave{T}) / s t d(T)
$$


where $T$ is turbidity and $\grave{T}$ the average and $s t d(T)$ the standard deviation of the turbidities in each data set. For $T_{z-s t d}$, six values between -0.5 to 8 (see Figure 12) were chosen to cover the turbidity range from close to the origin to far beyond the range of the calibration data pairs. For $T_{z-s t d}=2$ (typically the $95 \%$ percentile of the turbidities in the calibration data sets) RS increases significantly with decreasing $R^{2}$ from nearly $0 \%$ for $R^{2}$ close to 1 , to $10 \%$ for $R^{2}=0.93$ and $30 \%$ for $\mathrm{R}^{2}=0.60$. For $T_{z-s t d}=0$ (centre of the turbidities in the calibration data sets), RS is close to zero, as expected. For $T_{z-s t d}=8$ and $\mathrm{R}^{2}<0.6, \mathrm{RS}$ amounts to more than $50 \%$. Figure $12 \mathrm{~b}$ and $\mathrm{c}$ display slopes and intercepts by linear regression (Eigenvalues method) of RS for the six $T_{z-s t d}$. Their dependence can be closely fitted by exponentials and yields the following formula for RS as a function of $\mathrm{R}^{2}$ and $T_{z-s t d}$ :

$$
R U=140\left(e^{-0.35 T_{z-s t d}}-135\right) R^{2}+140\left(1-e^{-0.44 T_{z-s t d}}\right)-5
$$

This formula predicts the percentage spreading within $5 \%$ for an $R^{2}$ of 0.91 and can be used to estimate the uncertainties involved with the choice of the regression method applied based only on $\mathrm{R}^{2}$ and dependent on the value of $T_{z-s t d}$ for any data set.

We further investigated RS of each regression method for the exchange of independent and dependent variables, as this, according to Figure 9 , is the main control for the magnitude of RS. For each data set, we created the mean of all seven linear scaled data regressions as a baseline and computed for each regression method the Regression model choice Uncertainty (RU), defined as the relative differences of SPMC to the baseline value as a function of $T_{z-s t d}$. In Figure 13a RU averaged over the data sets with $\mathrm{R}^{2}<0.75$ and in Figure $13 \mathrm{~b} \mathrm{RU}$ averaged over the data sets with $\mathrm{R}^{2}>0.90$ versus $T_{z-s t d}$ is shown. At $T_{z-s t d}=0$, $\mathrm{RU}$ is nearly zero for all methods, whereas towards negative $T_{z-\text { std }}$ it diverges sharply reaching more $\pm 40 \%$ at $T_{z-s t d}=-1$ (the $10 \%$ percentile of the turbidities in the calibration data set), as the computed absolute SPMC differences in the denominator of $\mathrm{RU}$ are approaching zero. With increasing $T_{z-s t d}$, these differences remain confined to $\pm 15 \%\left(R^{2}<0.75\right)$ and $\pm 5 \%\left(R^{2}>0.9\right)$ within the calibration data range below $T_{z-s t d}=1.6$ (90\% percentile). However, further increasing $T_{z-s t d}$ results in even higher $\mathrm{RU}$ that depends both of the $\mathrm{R}^{2}$ of the data set and the regression method. For data sets with $R^{2}>0.90$, this plateau remains below $\pm 5 \%$ for all regression methods, but raises to nearly $\pm 20 \%$ for data sets with $R^{2}<0.75$. Here, the regression methods differentiate distinctively from each other, see Figure 13a. The LSQ method for example, with turbidity as dependent variable and SPMC computed from the inverse of the regression, deviates from the baseline by $+20 \%$, whereas the Eigenvalue and inverse robust fit regression still remain close to $+5 \%$. All inverse regressions have a positive bias with respect to the mean, whereas the regressions with SPMC as dependent variable have a negative bias of the same amount. 
The situation is quite different for the regressions performed with $\log _{10}$-transformed data. The slope of the regression may deviate from 1, resulting in a non-linear power function after retransformation. This may result in quite large $\mathrm{RU}$ at high $T_{z-s t d}$, see Figure 14. For data sets with $\mathrm{R}^{2}<0.75$, the $\mathrm{RU}$ exceeds by more than $\pm 50 \%$ at $T_{z-\text { std }}=8$ with no signs to reach a plateau limit at higher $T_{z-s t d}$. For $\mathrm{R}^{2}>0.9$, the $\mathrm{RU}$ is much lower with $\pm 20 \%$ at most and reaching the plateau at $T_{z-s t d}=6$. This value still is four times higher than for the regressions with nottransformed data. Within the calibration data range the uncertainties are still higher by a factor of 2 compared to the not transformed cases. At the lower end of the of $T_{Z-s t d}$, the RU of all models are more or less identical and remain low as all regressions are forced to cross the origin point.

Of course, the results shown here are valid for any data set independent of the meaning of the variables. This means that the uncertainties involved with the model calibration of acoustical backscatter data against an OBS derived $10 \log _{10}$ (SPMC) are much larger as the examples in Figure 10 and 11 show, where the $R^{2}$ is 0.4 and 0.6 respectively. The situation is even aggravated after raising the data to the power of 10 , where the uncertainties will increase accordingly and likely exceed $100 \%$.

\subsubsection{Variabilities in SPM inherent properties}

Variabilities in IOPs may occur between different geographical areas or within a same measuring location. The former is illustrated in Figure 15, where the relation between sample SPMC and Seapoint turbidity is shown for different areas in the North Sea. The spreading in the data is caused by variabilities in inherent particle properties between the areas. Additional causes of the differences are inter-sensor variabilities and uncertainties due to the sampling and filtration protocols. The latter are thought to be of lesser importance as the differences in slope of the regression lines (German Bight: 1.05 and Oosterschelde estuary: 2.34) is larger than these additional uncertainties. Local variation in the IOPs have thus to be considered when regional turbidity data are compiled into SPMC maps.

Intra-tidal variabilities in IOPs at a same location have been observed at various locations. Figure 16a shows that the specific backscatter ratio (i.e. turbidity divided by sample SPMC) is oscillating with the tides (Becherer et al., 2016) and that this may be largely assigned to the organic content of the particles that increases with the specific backscatter ratio (Figure 16b). The magnitude and the tidal signal of the ratio nearly persisted after the storm even though sample SPMC at maximum currents increased threefold. With respect to the tidal mean this would result in a systematic over- and underestimation of the SPMC by $60 \%$ over the tidal cycle. Variation in the specific backscatter ratio may also occur on seasonal time scales as observed in Liverpool Bay (Jafar-Sidik et al., 2017). These variations are caused by the 
seasonality of the primary production and turbulence regime and have changed the ratio by a factor 2 .

Another example shows intra-tidal variations in acoustical and optical inherent particle properties due to occurrence of mixed particles (i.e. sand and mud) in suspension at a muddy and sandy bed site located in the main tidal channel of a brackish marsh in the Scheldt estuary (Schwarz et al., 2017). The optical sensor was calibrated with sample SPMC and the optical derived SPMC was subsequently used to calibrate the acoustic backscatter sensor. The muddy bed site shows a strong correlation in contrast with the sandy bed site between acoustical and optical backscatter during the two tidal cycles (Figure 17). These observations indicate constant particle properties during a tide at the muddy site and changing particle properties at the sandy site, indicating that the SPM at the muddy site consists mostly of muddy flocs resuspended from the bed, while at the sandy site sand grains with diameters comparable to those of flocs are eroded into suspension at high flow velocities. Their intermittent abundance will lead to changes in the IOP's of the SPM due to their higher specific density, i.e. the increase of $r \rho$ will lower the ratio of turbidity to sample SPMC (see eq. 1). A storm event recorded at an observation pole in the Elbe river (Baschek et al., 2017, Kappenberg et al., 2018), discloses a further example of significant changes in this ratio due to sand grains entrained into the water column. The ratio of optical turbidity to SPMC derived from water samples taken hourly over a period of 12 hours before, during and after the storm varied from 0.4 during the moderate wind phases to 0.12 just after the peak of the wind speed (Figure 18). Grain-size analysis of the samples revealed a significant increase of mainly sand around the $90 \mu \mathrm{m}$ fraction. As a consequence, optical turbidity remained in the range of average tidal variability during the storm, whereas sample SPMC exceeded this by a factor of five. Similar observations have been made at other locations where mixed sediments and/or strong changing current and wave conditions caused sandy material or particle with other erosion characteristics to be resuspended up to the detection volumes of the acoustic and optical sensors (see e.g. Fugate and Friedrichs, 2002; Fettweis et al., 2012).

The sample SPMC and the sensor data should preferably be collected in situ as it represents the actual inherent properties of the SPMC and is thus more reliable than in laboratory model calibrations (ISO, 2014). In case no in situ samples can be collected or if the range of sample SPMC is too small, in laboratory model calibration can be applied with representative samples. Bollen et al. (2006) describes a laboratory model calibration using a representative in situ bed sample taken near the monitoring station. The bed sample was sieved on a $63 \mu \mathrm{m}$ sieve, followed by desalination and drying, to finally produce standard solutions with defined SPMC. The regression model using in situ data has a larger slope and overestimates the sensor output 
Version: August 11, 2019;

by about $10 \%$. The difference is most probably caused by changes of the laboratory versus in situ IOP of the SPM.

The examples demonstrated that in situ calibration with samples is necessary at all representative locations and phases of the local hydrodynamic and biogeochemical conditions to take the varying composition of the suspended particles into account.

\subsubsection{Comparison between optical and acoustical derived SPMC}

A comparison of the modelled SPMC was performed from different optical and acoustical sensors, based on the data from the mouth of the Seine estuary (Figure 10). The same sample SPMC was used to build models for a moored Wetlabs OBS, a profiling OBS3+ and a moored ADCP (1MHz Nortek AWAC) and the modelled optical derived SPMC was further used as reference SPMC to build a model for the moored ADCP in order to estimate and compare model performance and associated uncertainties (Figure 19). Results show that the optical sensors have highest accuracy against sample SPMC (OBS3+: 10\%, Wetlabs OBS: 40\%) and that the acoustical derived SPMC compare well with the OBS3+ profiles for the period where both sensors have been calibrated against sample SPMC. In the same figure the acoustic derived SPMC is compared with the SPMC time series derived from the Wetlabs OBS's, thus for periods that are not covered by the field campaigns dedicated for calibration. The optical and acoustic derived SPMC values significantly differ, the ADCP underestimating large optical derived SPMC and overestimating the lower ones. The acoustic sensor has the larger uncertainty at lowest (400\% for SPMC $<5 \mathrm{mg} / \mathrm{l}$ ) and highest values; in the mid-range the uncertainty is about $100 \%$ (5 $\mathrm{mg} / \mathrm{l}<\mathrm{SPMC}<10 \mathrm{mg} / \mathrm{l}$ ) and below $50 \%$ (SPMC $<75 \mathrm{mg} / \mathrm{l}$ ). The results also show that the error of the reference SPMC propagates into the uncertainty of the acoustic derived SPMC, as can be seen when comparing the ADCP derived SPMC from Wetlabs OBS (20-150\%) and OBS3+ (20$50 \%)$.

High uncertainties between acoustic and OBS derived SPMC have been observed in the data from the Belgian nearshore, see Figure 20. The normalized RMSE associated with the regression model varies between $100-500 \%$ (mean regression is 170\%) for the M284 ADP and 90-140\% (mean regression is 90\%) for the M947 ADP. The uncertainty is lowest in the mid-range SPMC (100-1000 mg/l), with normalized RMSE between 50-70\% (M284) and 45-60\% (M947) for the best regression models and higher for the lower and higher SPMC values.

Both examples indicate that the differences between acoustic and optic derived SPMC can be very large and that these uncertainties increase when long time series are used for calibration. These differences are probably caused by variabilities in inherent acoustical and optical properties of the SPM that occurred over time scales longer than the sampling surveys, by differences in built-in sensor technology or due to time-delay between sensor measurements 
Version: August 11, 2019;

and sample time. Further differences may also be caused by the presence of coloured dissolved organic matter (CDOM) that affects the optical measurements (Bright et al., 2018) and that may change the size and density of the flocs and thus the inherent properties of the particles (Lee et al., 2019). In order to improve the correlation, the inherent acoustic and optical properties of the particles, and the presence of dissolved OM should be incorporated into the model calibrations.

\subsection{Additional uncertainties}

Additional uncertainties are caused by the direct and indirect human interference in the chain of operation. These includes errors directly caused by the human variability, by the specific problems associated with working in the sea or by the effects of human activities not related to the operational chain that have an impact on the SPMC. Many of these errors are often only detected after the field campaign and should therefore be discarded, see for example the occurrence of outliers in the model parameter calibration that cannot be explained by natural processes. 
Version: August 11, 2019;

\section{Towards best practice}

To come to best practice for long-term monitoring activities of SPMC an accurate documentation of the successive operations and of the accumulating effect of uncertainties is required. An overview of the generic succession of operations required to run long-term or large-scale measurements of SPM concentrations is depicted in Figure 21. In long-term observations the pre-measurement, measurement and post-measurement phases and activities are not in strict sequence but interleave.

Beforehand knowledge on the magnitudes in SPMC will guide the choice of instrumentation and define the need for adjoined measurements of state variables such as particle size, shape and composition, salinity, temperature, current velocity or turbulence, see Table 2 . Before field usage, all sensors should be calibrated in the laboratory against standards to manifest a reference against any possible changes in sensor response after recovery. During the measurement phase the observing system is impacted by processes that may disturb the sensor outputs with respect to the preceding sensor and model calibration, as presented in chapter 3. A functional understanding of the interrelation between inherent particle properties, system state conditions and sensor SPMC will only be achieved if sufficient surveys are carried out to estimate the source and magnitude of changes in the sensor SPMC between the in situ calibration activities.

Post-measurement phase starts with the raw data retrieval and their conversion into asciibased data and archiving information that collate all so far undertaken calibration, accuracy and precision measures (Tzeng et al., 2016). After recovery from the field, optical and (if possible) acoustical sensors should be calibrated against the lab reference to quantify any changes in sensor response due to electronic drift or optical window damages. In post-processing the data should first undergo an expert judgement to flag occurrences of spikes, stuck values or unreliable magnitudes. The resulting set of accepted and corrected data together with sample SPMC acts as input for the computation of the sensor SPMC. The associated error estimates are due to the measurement procedures themselves and yield only the errors at the observing point and for the calibration periods of the time series.

\subsection{Discussion and ranking of the uncertainties}

The overall error of the SPMC data set consists of random errors that lead to uncertainties of individual SPMC but approximate the accurate value with increasing amount of data; and systematic errors (biases) that lead to an average over- or underestimation of all data. There exist two types of biases: one that is constant or independent of observing times and locations (e.g. the choice of the regression method, as discussed in section 3.4.2) and another one that changes with time and location (section 3.4.3). Some kinds of errors can be detected, and to 
Version: August 11, 2019;

some extent corrected, whereas, others are inherently associated with the applied technologies and its interference with the environment and remain spurious and difficult to quantify or to control. The first types of errors are related to the sensors, sampling and lab protocols or the modelling techniques, while the latter are mainly related to systematic, often gradually changing natural variability in SPM inherent properties. Table 3 lists the sources of uncertainties as described in chapter 3 . The listed uncertainties represent typical values that indicate their relative importance for the overall error. The following discussion continues along the vertical order of table 3 and relates to the major items of the workflow depicted in Figure 21. As the errors and their mitigation or correction measures are quite dependent on the site conditions, we did not give a set of specific guidelines. In our view, regular sensor calibration against a standard solution, field inter-calibrations of sensors against reference sensors, biofouling, splitting up the sample into different volumes for filtration at SPMC $<3 \mathrm{mg} / \mathrm{l}$, the choice of the regression model and long-term changes in the IOPs are the most critical elements in the workflow.

\subsubsection{Sensor related uncertainties}

Most sensor related sources of uncertainty can be deduced from the correct ratio of the sensor signal to a standard solution. Any deviation from the 1:1 ratio results in a bias of potentially $\pm 15 \%$ that will pass through the whole workflow and needs to be detected and corrected for. Controlling the sensor drift will require either the close follow up of the battery voltage and indirect field inter-calibration against a well calibrated reference sensor operated in parallel very close to the long-term observing location, or by regular re-calibration checks in the laboratory with formazine or other reference solutions after recovery of the sensor.

The usage of sensors that are not adapted to the range of turbidity values of the site, as shown in section 3.1.2, may reduce the overall accuracy of the output in general by a bias of about $2 \%$ up to $24 \%$. The limited turbidity resolution may also increase the random or steady changes in IOPs and thus in the ratio of sample SPMC to turbidity.

The comparison of the SPMC derived from different OBS3+ sensors (section 3.1.3) shows that inter-sensor variability for identical sensor types can be reduced to natural random fluctuations in the water bodies, if all sensors are calibrated separately against sample SPMC. If intercomparing different types of sensors, it is not evident beforehand that even separate calibration leads to the same results for longer time series, as different sensors may react differently to changes in the IOPS of the SPM. This may result in time-dependent biases and has to be investigated case by case.

\subsubsection{Environmental related uncertainties}


Biofouling represents an important challenge for accurate measurements of turbidity and in some cases of acoustical backscatter intensity in the field. If not detected and flagged, the bias (positive for turbidity; negative for acoustical backscatter) can easily reach many tens of percent. Especially optical sensor output is sensitive to increasing coverage of the sensors material disturbing the light pathways or scatter detection volume. Although technologies exist to mitigate the impact of fouling, regular cleansing of the sensors remains a necessity and needs to be accompanied by sensor inter-calibration against a reference device or solution. As long as the effect of biofouling is limited, corrections by means of independent not-affected devices is sometimes possible. In many cases, however, tagging and discarding bio-fouled data is the only option.

Discarding the very high SPMC that saturate the sensor output will introduce a bias. Modelling the gaps by extending the power spectrum of the time series will lead to more realistic values, however, the degree of uncertainty depends on the specific situation and the applied interpolation model.

\subsubsection{Sample related uncertainties}

The determination of sample SPMC is through three steps, sampling, filtering and subsequent weighing. The main uncertainties involved are related to the filtering procedure at SPMC $<3$ $\mathrm{mg} / \mathrm{l}$, because the weights of the filter loadings are in the order of the sample specific filter offset. So far the most accurate protocol to determine this offset is the method with different filter volumes by Röttgers et al. (2014). At SPMC $>25 \mathrm{mg} / \mathrm{l}$ the error without knowing the filter offset amounts to $3 \%$, but raises to $50 \%$ for SPMC $<3 \mathrm{mg} / \mathrm{l}$. When the filter offset is known then the uncertainties reduce to less than $10 \%$ for SPMC $<3 \mathrm{mg} / \mathrm{I}$. For SPMC $>25 \mathrm{mg} / \mathrm{l}$ the impact of the filter offset becomes negligible. Using only one filter in this range bears the risk that faulty sample SPMC cannot be identified and show up as outliers in the scatter plots that cannot be removed from the calibration data set. This can be avoided by working with triplicates and removing the sample that differs from the other two by more than a pre-defined value which reduces the random error by a factor of 2 down to the order of $5 \%$ or less.

A simple method to evaluate the quality of the sample SPMC compared with in situ optical measurements consist in using a Hach turbidimeter in addition to the filtration. Large inconsistency between the Hach sample turbidity measured on board, the in situ turbidity from a sensor and the sample SPMC will designate this sample as unreliable.

\subsubsection{Choice of the linear regression model}

The choice of the linear regression model including the choice of the dependent and independent variable has some significance as it is systematic and leads to SPMC that are too high or too low. To our knowledge, publications involving SPMC estimated from optical 
turbidity or acoustical backscatter do not consider this issue. It is quite evident and quantified in Figures 13 and 14 that a calibration data set within an $R^{2}>0.9$ should be achieved to keep uncertainties involved with the choice of a specific regression model well below $10 \%$, independent of the model and the range of the calibration data compared to the complete data set. However, under circumstances of variable IOPs or IAPs in the sample calibration period, such a high $\mathrm{R}^{2}$ may not be achievable.

From the three regression methods that are sensitive to the exchange of dependent and independent variables, it is the Robust fit that in our examples shows the least sensitivity to this exchange, followed by the Theil-Sen estimator and the LSQ method as exemplified by Figures 13 and 14 . A recommendation for the best, i.e. least sensitive regression model is not straightforward. The reason is that although prominent outliers in the calibration data set may suggest the preference for a robust method, they bear the risk of creating biases in the regression if they are caused by erratic variations in the inherent particle properties (see section 3.4.3) and not by sensor, sampling or human related faults. In the absence of substantial, not removable outliers, Eigenvalue regression may be the proper option, as both SPMC and turbidity/acoustical backscatter, averaged over the sampling period, have errors of comparable magnitude. As another option, the regression average of a method with exchanged ordinate and abscissa may be used. The random error statistics of this approach may be generated by a boot-strap method. Regressions with $\log _{10}$-transformed data exhibit a generally higher sensitivity to the model choice for $T_{z-s t d}>0$ that increase substantially for turbidities above the calibration data range. In this respect they bear the risk of substantial biases in the SPMC computed from higher optical or acoustical backscatter data.

\subsubsection{Variabilities in SPM inherent optical and acoustical properties}

Uncertainties due to undetected changes in inherent particle properties of the SPM are difficult to quantify during long-term SPMC measurements, however, they may be significant for specific sites or periods. All of them can be ascribed to changes in particle size, shape, composition and density and to the occurrence of coloured dissolved organic substances. Regular water sampling to compare the sample SPMC with sensor output will provide insight into the stability of models used to estimate sensor SPMC over time and/or space (e.g. Druine et al., 2018). The examples of section 3.4.3 show that even intra-tidal variations or events of several hours lead to systematic uncertainties of $50 \%$ to $200 \%$. Therefore, it is recommended to add further sensors to the observing system that give direct indication for changes in the inherent particle properties (Table 2).

When measuring in the benthic boundary layer the SPMC may reach values that exceed the calibration data range. The calibration data set does not cover the sensor SPMC data range and 
may result in high uncertainty for the largest SPMC value. For these situations a model calibration in the laboratory allows extending the calibration data range (see section 3.4.3). This is meaningful if the associated uncertainty is lower than the one from an in situ model calibration at large $T_{Z-s t d}$.

\subsection{Water sample turbidity as a surrogate for SPMC?}

\subsubsection{Uncertainty between SPMC and turbidity}

To separate variability due to measurement errors from variability due to natural variations in IOPs, protocols that use the same water sample for filtration and for turbidity estimation should be used in parallel to the in situ procedures. Figure 22 shows a collection of sample SPMC versus turbidity measurements of the same water sample recorded with a portable ISO Hach 2100P nephelometer from various waters around the world (North Sea, English Channel, Mediterranean Sea, Rio de la Plata, French Guiana nearshore, Gironde estuary, Scheldt estuary, see Table 3), collected by the RBINS (Dogliotti et al., 2015; Knaeps et al., 2015) and HZG (Röttgers et al., 2014). The protocols for sample SPMC and turbidity measurements are those described in Neukermans et al. (2012a) for RBINS and Röttgers et al. (2014) for HZG. The figure shows that the ratio between sample SPMC and Hach turbidity is quite stable within an uncertainty of $20 \%$ throughout the regions. In this collection, $4.1 \%$ of couples fail outside of the 95\% confidence interval regarding the t-distribution of sample SPMC/turbidity in the logarithmic space. The outlying couples probably reflect the tails of the combined natural variability of particles mass-specific side-scattering coefficient, and sampling and filtration errors. The average ratio of sample SPMC to turbidity from all data is about $1.13 \mathrm{mg} / \mathrm{I} / \mathrm{FNU}$ (RBINS data), and $1.19 \mathrm{mg} / \mathrm{l} / \mathrm{FNU}$ (HZG data).

Despite calibration to a formazine reference solution and the use of ISO-normed optical sensors, model calibration may vary considerably in recorded turbidity for a same SPMC solution across different instruments resulting in instrument-specific turbidity-SPMC relations, see Figure 23. Although different types of optical sensors have been calibrated against the same reference solution, they yield up to $20 \%$ differences results in the field. Another example concerns data collected by Ifremer and the University of Rouen at different locations in the Seine estuary (Druine et al., 2018) with a ISO Hach $2100 \mathrm{~N}$ nephelometer (same constructor, but different type as the above mentioned 2100P) give significant different turbidity values for the same SPMC. Given the consistency of the Hach 2100P data pairs from two institutes over a wide range of areas we assume that the differences are mainly caused by the instrument specificities. The latter was confirmed during inter-calibration of both ISO standardized nephelometers with StabICal ${ }^{\circledR}$ and with SPMC solutions. The inter comparison revealed that the $2100 \mathrm{~N}$ measures about $13 \%$ lower turbidity values for the same StablCal ${ }^{\circledR}$ solution than the 
Version: August 11, 2019;

2100P. This strengthens further that any turbidity unit should be referred to the instrument used and the protocol applied (see section 2.1).

\subsubsection{Need for further sample SPMC?}

The above results prohibit the comparison of turbidity values measured by different types of optical sensors. Turbidity as a surrogate of SPMC is only reliable, as long as site-specific (model) and instrument-specific (sensor) calibrations are carried out. On the other hand, the quiet stable relationship between the ISO Hach water sample turbidity and the sample SPMC in Figure 22 throughout different localities suggests that this relationship could be used as an estimate of SPMC in two ways. Firstly, it adds a third independent measurement and helps to separate model-calibration outliers due to natural fluctuations from sample or human faults. Secondly, the stable ratio of Hach derived turbidity to sample SPMC could be used instead of sample SPMC for in situ turbidity data. This introduce an individual random uncertainty of some $20 \%$, which could be compensated by less laboratory effort and therefore more available calibration data. The site specific regressions for sample SPMC versus Hach turbidity vary by only $20 \%$ in the RBINS data set shown in Figure 22 . Using the global fit at different sites still reproduces the sample SPMC within less than $20 \%$ with an average bias of $3 \%$. Especially given the high uncertainties of low sample SPMC due to the filter offsets, a $20 \%$ random error for Hach-derived SPMC seems to be a suitable and cheap alternative to filter weights. 


\section{Conclusions and Outlook}

Optical and acoustical sensors have proved invaluable in the study of SPM dynamics in marine and estuarine environments as they allow collecting easily in situ, high-frequency SPMC time series over long periods of time. The payback is the availability of large homogeneous data set of SPMC from various locations on the globe; the drawback is that the quality or certainty of the data and thus also the inter-comparability depends on factors that are only to a certain level avoidable.

Long-term observations of SPMC are the result of a complex ladder of operations that involve field, laboratory and modelling methods. Each step contributes its own random and systematic errors to the overall uncertainties of the sensor SPMC. Systematic errors related to the functioning of the sensors, the environment, the collection and processing of calibration samples and faulty human operations are detectable and sometimes correctable. As long as protocols for sample analysis and sensor calibration are carefully obeyed, uncertainties can be confined within $\pm 5 \%$, otherwise they may reach up to $\pm 20 \%$. Biofouling may add a further bias of $100 \%$ (positive for optical, negative for acoustical sensors), and their detection in generally leads to a loss of data. A good understanding of the processes that are causing changes in SPMC and particle inherent properties (size, shape, density and composition) is required in order to estimate their importance and to possibly rescale the sensor data to some reference particle properties. Variations in these properties may result in over- or underestimation of the SPMC by up to a factor 2 or more. Based on the uncertainties, listed in table 3, one can achieve random errors below $25 \%$ and biases below $40 \%$ only with substantial efforts in technologies that indicate the changes in inherent particle properties.

Acoustical and optical sensors require both the conversion of the sensor output (after sensor calibration) to a mass concentration. This is done by relating the sensor output to a reference SPMC, which is preferably the sample SPMC. The choice of the regression method, the dependent and independent variable, and the error associated with the reference SPMC determines the coefficient of determination. We have built a model that based on the $\mathrm{R}^{2}$ and the normalized turbidity/dB quantifies the uncertainty of the sensor derived SPMC in the calibration range and outside of it. The model shows that the Robust fit and the Eigenvalue regression have less prediction bias than the Theil-Sen estimator and the ordinary LSQ. This bias is not an issue for $\mathrm{R}^{2}>0.9$ and remains below $10 \%$, but it becomes significant for lower $\mathrm{R}^{2}$ and can amount to $30 \%$. Short-term variabilities in the model-regressions generally show up as random noise limiting the $\mathrm{R}^{2}$ of the calibration data set, but the extrapolation of the regression parameters to longer periods or larger areas may introduce biases of more than $50 \%$. 
Version: August 11, 2019;

The site specific regressions for sample SPMC versus Hach turbidities vary by $20 \%$ between different coastal regions and even less within the same region. This relationship could be a cheap alternative to filter weights as less laboratory effort is needed as long as the same nephelometer is used. Indeed, our study confirms that the relation between turbidity and sample SPMC is depending on protocols (EPA, ISO), technology (scattering angle) and the manufacturer, and even may differ between sensors of the same type (e.g. Downing, 2006; Rai and Kumar, 2015; Rymszewicz et al., 2017). The relation between the output of an acoustical sensor and SPMC is even more variable. In spite of these uncertainties, turbidity is still often used as a proxy for water clarity or SPMC as is the $\mathrm{dB}$ of acoustical sensors. We advise to not use turbidity (or $\mathrm{dB}$ ) for scientific purposes as it diminishes the comparability of the data. Instead, the sensor output should be transformed into a mass concentration, a unit that is comparable in time and between regions. If this is not possible, then the turbidity data should always be referred to the instrument used and the protocol applied. The problem aggravates when turbidity data that have been collected using different technologies and protocols over long periods of time and regional scales are stored in international data bases (e.g. turbidity in EMODnet, see http://www.emodnet.eu), and used to derive conclusive trends of the environmental status of marine and estuarine areas. We hope that the uncertainty analysis presented here will serve as a basis for the validation of historical turbidity and SPMC data and that it offers guidelines to obtain inter-comparable high quality long-term SPMC time series. 
Version: August 11, 2019;

\section{Acknowledgements}

This work was initiated on a meeting held in February 2016. As an outcome a number of recommendations were formulated that have been compiled in the text. The first four authors have structured compiled and written large parts of the text. The other authors have provided examples, data and have contributed to the writing. The research was supported by the Belgian Science Policy (BELSPO) within the BRAIN-be program (INDI67 project), the Maritime Access Division of the Flemish Ministry of Mobility and Public Works (MOMO project), the research and development programme of the Federal Waterways Engineering and Research Institute (BAW) and the Seine Aval Research Program (SUSPENSE and PHERSQUES projects). Ship time for the RBINS data collection was provided by BELSPO and RBINS-OD Nature. 


\section{References}

Abarzua, S., Jakubowski, S., 1995. Biotechnological investigation for the prevention of biofouling. I Biological and biochemical principles for the prevention of biofouling. Marine Ecology Progress Series 123, 301-312.

Adriaens, R., Zeelmaekers, E., Fettweis, M., Vanlierde, E., Vanlede, J., Stassen, P., Elsen, J., Środoń, J., Vandenberghe, N., 2018. Quantitative clay mineralogy as provenance indicator for recent muds in the southern North Sea. Marine Geology 398, 48-58. doi:10.1016/j.margeo.2017.12.011

Agrawal, Y.C., Pottsmith, H.C., 2000. Instruments for particle size and settling velocity observations in sediment transport. Marine Geology 168, 89-114.

Ainslie, M.A., McColm, J.G., 1998. A simplified formula for viscous and chemical absorption in sea water. Journal of the Acoustical Society of America 103, 1671-1672.

Anastasiou, S., Sylaios, G.K., Tsihrintzis, V.A., 2015. Suspended particulate matter estimates using optical and acoustic sensors: application in Nestos River plume (Thracian Sea, North Aegean Sea). Environmental Monitoring and Assessment 187, 1-17. doi:10.1007/s10661-015-4599-y

Babin, M., Morel, A., Fournier-Sicre, V., Fell, F., Stramski, D., 2003. Light scattering properties of marine particles in coastal and open ocean waters as related to the particle mass concentration. Limnology and Oceanography 48, 843-859. doi:10.4319/lo.2003.48.2.0843

Badewien, T.H., Zimmer, E., Bartholomä, A., Reuter, R., 2009. Towards continuous long-term measurements of suspended particulate matter (SPM) in turbid coastal waters. Ocean Dynamics 59, 227-238. doi:10.1007/s10236-009-0183-8

Baeye, M., Fettweis, M., 2015. In situ observations of suspended particulate matter plumes at an offshore wind farm. Geo-Marine Letters 35, 247-255. doi:10.1007/s00367-015-0404-8

Baker, E.T., Lavelle, J.W., 1984. The effect of particle size on the light attenuation coefficient of natural suspensions. Journal of Geophysical Research 89 (C5), 8197-8203.

Banse, K., Falls, C.P., Hobson, L.A., 1963. A gravimetric method for determining suspended matter in sea water using Millipore filters. Deep Sea Research and Oceanographic Abstracts. 639-642.

Barillé-Boyer, A.-L., Barillé, L., Massé, H., Razet, D., Héral, M., 2003. Correction for particulate organic matter as estimated by loss on ignition in estuarine ecosystems. Estuarine, Coastal and Shelf Science 58, 147-153. doi:10.1016/S0272-7714(03)00069-6

Barter, P.J., Deas, T., 2003. Comparison of portable nephelometric turbidimeters on natural waters and effluents. New Zealand Journal of Marine and Freshwater Research 37, 485-492.

Baschek, B., Schroeder, F., Brix, H., Riethmüller, R., Badewien, T.H., Breitbach, G., Brügge, B., Colijn, F., Doerffer, R., Eschenbach, C., Friedrich, J., Fischer, P., Garthe, S., Horstmann, J., Krasemann, H., Metfies, K., Merckelbach, L., Ohle, N., Petersen, W., Pröfrock, D., Röttgers, R., Schlüter, M., Schulz, J., Schulz-Stellenfleth, J., Stanev, E., Staneva, J., Winter, C., Wirtz, K., Wollschläger, J., Zielinski, O., Ziemer, F., 2017. The Coastal Observing System for Northern and Arctic Seas (COSYNA). Ocean Sciences 13, 379-410. doi:10.5194/os-13-379-2017

Becherer, J., Flöser, G., Umlauf, L., Burchard, H., 2016. Estuarine circulation versus tidal pumping: Sediment transport in a well-mixed tidal inlet. Journal of Geophysical Research 121, 6251-6270. doi:10.1002/2016JC011640

Becker, M., Schrottke, K., Bartholomä, A., Ernstsen, V., Winter, C., Hebbeln, D., 2013. Formation and entrainment of fluid mud layers in troughs of subtidal dunes in an estuarine turbidity zone. Journal of Geophysical Research 118, 2175-2187. doi:10.1002/jgrc.20153

Berthon, J.-F., Shybanov, E., Lee, M.E.-G., Zibordi, G., 2007. Measurements and modeling of the volume scattering function in the coastal northern Adriatic Sea. Applied Optics 46, 5189-5203. doi:10.1364/A0.46.005189

Binding, C.E., Bowers, D.G., Mitchelson-Jacob, E.G., 2005. Estimating suspended sediment concentrations from ocean colour measurements in moderately turbid waters; the impact of variable particle scattering properties. Remote sensing of Environment 94, 373-383. doi:10.1016/j.rse.2004.11.002

Bolanos, R., Amoudry, L., Doyle, K., 2011. Effects of instrumented bottom tripods on process measurements. Journal of Atmospheric and Oceanic Technology 28, 827-837. doi:10.1175/2010JTECHO816.1

Bollen, M., Sas, M., Vanlede, J., de Mulder, T., 2006. Measuring High Concentration Benthic Suspensions (HCBS), using a high resolution SiltProfiler. Proc. $15^{\text {th }}$ International Congress of the International Federation of Hydrographic Societies. Special Publication, 55, 138-140. www.vliz.be/imisdocs/publications/114165.pdf 
Boss, E., Pegau, W.S., 2001. Relationship of light scattering at an angle in the backward direction to the backscattering coefficient. Applied Optics 40, 5503-5507. doi:10.1364/AO.40.005503

Boss, E., Pegau, W.S., Lee, M., Twardowski, M.S., Shybanov, E., Korotaev, G., Baratange, F., 2004. The particulate backscattering ratio at LEO 15 and its use to study particle composition and distribution. Journal of Geophysical Research 109(C01014). doi:10.1029/2002JC001514

Boss, E., Taylor, L., Gilbert, S., Gundersen, K., Hawley, N., Janzen, C., Johengen, T., Purcell, H., Robertson, C., Schar, D.W.H., Smith, G.J., Tamburri, M.N., 2009a. Comparison of inherent optical properties as a surrogate for particulate matter concentration in coastal waters. Limnology \& Oceanography Methods 7, 803-810. doi:10.4319/lom.2009.7.803

Boss, E., Slade, W., Hill, P. 2009b. Effect of particulate aggregation in aquatic environments on the beam attenuation and its utility as a proxy for particulate mass. Optic Express 17, 9408-9420. doi:10.1364/OE.17.009408

Bowers, D.G., Binding, C.E., 2006. The optical properties of mineral suspended particles: a review and synthesis. Estuarine, Coastal and Shelf Science 67, 219-23. doi:10.1016/j.ecss.2005.11.010

Bowers, D.G., Braithwaite, K.M., Nimmo-Smith, W.A.M., Grahams, G.W., 2009. Light scattering by particles suspended in the sea: The role of particle size and density. Continental Shelf Research 29, 1748-1755 doi:10.1016/j.csr.2009.06.004

Bowers, D.G., McKee, D., Jago, C.F., Nimmo-Smith, W.A.M. 2017. The area-to-mass ratio and fractal dimension of marine flocs. Estuarine, Coastal and Shelf Science, 189, 224-234. doi:10.1016/j.ecss.2017.03.026

Bright, C.E., Mager, S.M., Horton, S.L. 2018. Predicting suspended sediment concentration from nephelometric turbidity in organic-rich waters. River Research and Applications 34, 629-639. doi:10.1002/rra.3305

Butman, B., Noble, M., Folger, D.W., 1979. Long-term observations of bottom current and bottom sediment movement on the mid-Atlantic continental shelf. Journal of Geophysical Research 84(C3), 1187-1205. doi:10.1029/JC084iC03p01187.

Cartwright, G.M., Friedrichs, C.T., Dickhudt, P.J., Gass, T., Farmer, F.H. 2009. Using the Acoustic Doppler Velocimeter (ADV) in the MUDBED real-time observing system. OCEANS 2009.

Chami, M., Marken, E., Stamnes, J.J., Khomenko, G., Korotaev, G., 2006. Variability of the relationship between the particulate backscattering coefficient and the volume scattering function measured at fixed angles. Journal of Geophysical Research 111(C5), C05013. doi:10.1029/2005JC003230

Chapalain, M., Verney, R., Fettweis, M., Jacquet, M., Le Berre, D., Le Hir, P. 2019. Investigating suspended particulate matter in coastal waters using fractal theory. Ocean Dynamics, 69, 59-81. doi:10.1007/s10236-0181229-6

Delauney, L., Compère, C., Lehaitre, M., 2010. Biofouling protection for marine environmental sensors. Ocean Science 6, 503-511. doi:10.5194/os-6-503-2010

Dogliotti, A.-I., Ruddick, K., Nechad, B., Doxaran, D., Knaeps, E., 2015. A single algorithm to retrieve turbidity from remotely-sensed data in all coastal and estuarine waters. Remote Sensing of Environment 156, 157-168. doi:10.1016/j.rse.2014.09.020

Dogliotti, A., Nechad, B., Ruddick, K., Gossn, J.I., 2016. Towards an autonomous turbidimeter network for multimission ocean colour satellite validation activities. In: Proc. Living Planet Symposium (ed. Ouwehand, L.), ESASP Volume 740, ISBN: 978-92-9221-305-3

Dolphin, T.J., Green, M.O., Radford J.D.J., Black, K.P., 2000. Biofouling of optical backscatter sensors: Prevention and analytical correction of data. Journal of Coastal Research, SI34, 334-341.

Downing, A., Thorne, P.D., Vincent, C.E., 1994. Backscattering from a suspension in the near field of a piston transducer. Journal of the Acoustical Society of America 97 (3), 1614-1620.

Downing, J., 2005. Turbidity Monitoring. In Environmental Instrumentation and Analysis Handbook (Eds. Down, R.D., Lehr, J.H.), John Wiley \& Sons Inc. doi:10.1002/0471473332.ch24

Downing, J., 2006. Twenty-five years with OBS sensors: The good, the bad, and the ugly. Continental Shelf Research 26, 2299-2318. doi:10.1016/j.csr.2006.07.018

Doxaran, D., Leymarie, E., Nechad, B., Dogliotti, A., Ruddick, K., Gernez, P., Knaeps, E., 2016. Improved correction methods for field measurements of particulate light backscattering in turbid waters. Optics Express 24(4), 36153637. doi:10.1364/OE.24.003615

Droppo, I., Leppard, G., Liss, S., Milligan T., 2005. Flocculation in Natural and Engineered Environmental Systems, CRC Press, Boca Raton, Fla, 438pp. 
Druine, F., Verney, R., Deloffre, J., Lemoine, J.-P., Chapalain, M., Landemain, V., Lafite, R., 2018. In situ high frequency long term measurements of suspended sediment concentration in turbid estuarine system (Seine Estuary, France): Optical turbidity sensors response to suspended sediment characteristics. Marine Geology, 400, 24-37. doi:10.1016/j.margeo.2018.03.003

Duan, N., 1983. Smearing estimate-A nonparametric retransformation method: Journal of the American Statistical Association 78 (383), 605-610.

Dufois, F., Verney, R., Le Hir, P., Dumas, F., Charmasson, S., 2014. Impact of winter storms on sediment erosion in the Rhone River prodelta and fate of sediment in the Gulf of Lions (North Western Mediterranean Sea). Continental Shelf Research 72, 57-72. doi:10.1016/j.csr.2013.11.004

Dyer, K.R., Manning, A.J., 1999. Observation of the size, settling velocity and effective density of flocs, and their fractal dimensions. Journal of Sea Research 41, 87-95.

Edwards, T.K., Glysson, G.D., 1999. Field methods for measurement of fluvial sediment. U.S. Geological Survey Techniques of Water-Resources Investigations, USGS Survey Book 3, chap. C2, 89 pp.

Eisma, D., 1986. Flocculation and de-flocculation of suspended matter in estuaries. Netherlands Journal of Sea Research 20, 183-199.

EPA, 1993. Method 180.1 - Determination of turbidity by nephelometry (revision 2.0), Environmental Protection Agency, Cincinnati, 10.

Fettweis, M., Sas, M., Monbaliu, J., 1998. Seasonal, Neap-spring and Tidal Variation of Cohesive Sediment Concentration in the Scheldt Estuary. Belgium. Estuarine, Coastal and Shelf Science 47, 21-36. doi:10.1006/ecss.1998.0338

Fettweis M., Francken F., Pison V., Van den Eynde, D., 2006. Suspended particulate matter dynamics and aggregate sizes in a high turbidity area. Marine Geology 235, 63-74. doi:10.1016/j.margeo.2006.10.005

Fettweis, M., 2008. Uncertainty of excess density and settling velocity of mud flocs derived from in situ measurements. Estuarine, Coastal and Shelf Science 78, 426-436. doi:10.1016/j.ecss.2008.01.007

Fettweis, M., Baeye, M., Lee, B.J., Chen, P., Yu, J.C.R., 2012. Hydro-meteorological influences and multimodal suspended particle size distributions in the Belgian nearshore area (southern North Sea). Geo-Marine Letters 32, 123-137. doi:10.1007/s00367-011-0266-7

Fettweis, M., Baeye, M., 2015. Seasonal variation in concentration, size and settling velocity of muddy marine flocs in the benthic boundary layer. Journal of Geophysical Research 120, 5648-5667. doi:10.1002/2014JC010644

Fettweis, M., Baeye, M., Cardoso, C., Dujardin, A., Lauwaerts, B., Van den Eynde, D., Van Hoestenberghe, T., Vanlede, J., Van Poucke, L., Velez, C., Martens, C., 2016. The impact of disposal of fine grained sediments from maintenance dredging works on SPM concentration and fluid mud in and outside the harbor of Zeebrugge. Ocean Dynamics 66, 1497-1516. doi:10.1007/s10236-016-0996-1

Fettweis, M., Lee, B.J., 2017. Spatial and seasonal variation of biomineral suspended particulate matter properties in high-turbid nearshore and low-turbid offshore zones. Water 9, 694. doi:10.3390/w9090694

Guerrero, M., Di Federico, V. 2018. Suspended sediment assessment by combining sound attenuation and backscatter measurements - analytical method and experimental validation. advances in Water Resources 113, 167-179. doi:10.1016/j.advwatres.2018.01.020

Fugate, D.C., Friedrichs, C.T., 2002. Determining concentration and fall velocity of estuarine particle populations using ADV, OBS and LISST. Continental Shelf Research 22, 1867-1886. doi:10.1016/S0278-4343(02)00043-2

Ganju, N.K., Schoellhamer, D.H., 2006, Annual sediment flux estimates in a tidal strait using surrogate measurements. Estuarine, Coastal and Shelf Science 69, 165-178. doi:10.1016/j.ecss.2006.04.008

Garel, E., Ferreira, O., 2011. Monitoring estuaries using non-permanent stations: practical aspects and data examples. Ocean Dynamics 61, 891-902. doi:10.1007/s10236-011-0417-4

Gibbs, R. J., 1985. Estuarine flocs: Their size, settling velocity and density. Journal of Geophysical Research 90 (C2), 3249-3251.

Gil, Y., David, C.H., Demir, I., Essawy, B.T., Fulweiler, R.W., Goodall, J.L., Karlstrom, L., Lee, H., Mills, H.J., Oh, J.-H., Pierce, S.A., Pope, A., Tzeng, M.W., Villamizar, S.R., Yu, X., 2016. Toward the geoscience paper of the future: Best practices for documenting and sharing research from data to software to provenance. Earth and Space Science, 3, 388-415, doi:10.1002/2015EA000136.

Gilbert, R.O., 1987. Statistical Methods for Environmental Pollution Monitoring. Van Nostrand Reinhold Company Inc. New York, 320pp. 
Gostiaux, L., van Haren, H., 2010. Extracting meaningful information from uncalibrated backscattered echo intensity data. Journal of Atmospheric and Oceanic Technology, 27, 943-949. doi:10.1175/2009JTECHO704.1

Grabemann, I., Krause, G., 1989. Transport processes of suspended matter derived from time series in a tidal estuary. Journal of Geophysical Research 94(C10), 14373-14379. doi:10.1029/JC094iC10p14373.

Gray, J.R., Gartner, J.W., 2009. Technological advances in suspended-sediment surrogate monitoring, Water Resources Research, 45, W00D29. doi:10.1029/2008WR007063

Guerrero, M., Di Federico, V., 2018. Suspended sediment assessment by combining sound attenuation and backscatter measurements - analytical method and experimental validation. Advances in Water Resources, 113, 167-179. doi:10.1016/j.advwatres.2018.01.020

Guézennec, L., Lafite, R., Dupont, J.P., Meyer, R., Boust, D., 1999. Hydrodynamics of suspended particulate matter in the tidal freshwater zone of a macrotidal estuary (the Seine Estuary, France). Estuaries 22, 717-727.

Ha, H.K., Hsu,W.-Y., Maa,J.P.-Y., Shao,Y.Y., Holland,C.W., 2009. Using ADV backscatter strength for measuring suspended cohesive sediment concentration. Continental Shelf Research 29, 1310-1316. doi:10.1016/j.csr.2009.03.001

Ha, H.K., Maa, J.P.-Y., Park, K., Kim, Y.H., 2011. Estimation of high-resolution sediment concentration profiles in bottom boundary layer using pulse-coherent acoustic Doppler current profilers. Marine Geology 279, 199-209. doi:10.1016/j.margeo.2010.11.002

Hatcher, A., Hill, P., Grant, J., Macpherson, P., 2000. Spectral optical backscatter of sand in suspension: effects of particle size, composition and colour. Marine Geology 168, 115-128. doi:10.1016/S0025-3227(00)00042-6

Hay, A.E., Sheng, J. 1992. Vertical profiles of suspended sand concentration and size from multifrequency acoustic backscatter. Journal of Geophysical Research 97, 15661-15677.

Helsel, D.R., Hirsch, R.M. 2002. Statistical Methods in Water Resources. Techniques of Water-Resources, Investigations of the US Geological Survey, Book 4, Hydrologic Analysis and Interpretation, 510pp. https://pubs.usgs.gov/twri/twri4a3/

HELCOM, 2015. Manual for Marine Monitoring in the COMBINE Programme of HELCOM, 466pp. http://helcom.fi/action-areas/monitoring-and-assessment/manuals-and-guidelines/combine-manual

Hoitink A.J.F., Hoekstraa, P., 2005. Observations of suspended sediment from ADCP and OBS measurements in a mud-dominated environment. Coastal Engineering 52, 103-118. doi:10.1016/j.coastaleng.2004.09.005

Holdaway, G.P., Thorne, P.D., Flatt, D., Jones, S.E., Prandle, D., 1999. Comparison between ADCP and transmissometer measurements of suspended sediment concentration. Continental Shelf Research 19(3), 421441. doi:10.1016/S0278-4343(98)00097-1

IOCCG, 2011. Bio-optical sensors on ARGO floats. In: Claustre, H. (ed.) Reports of the International Ocean-Colour Coordinating Group, No. 11, IOCCG, Dartmouth, Canada. http://www.ioccg.org/reports/IOCCG_Report11.pdf

IOC, 1996. Workshop Report No. 122, IOC-EU-BSH-NOAA-(WDC-A) International Workshop on Oceanographic Biological and Chemical Data Management, Intergovernmental Oceanographic Commission Hamburg, Germany, 20-23 May 1996

ISO, 1997. Water quality -- Determination of suspended solids by filtration through glass-fibre filters. ISO Standard 11923:1997, International Organization for Standardization, Geneva, Switzerland.

ISO, 1999. Water quality - determination of turbidity, ISO Method 7027, International Organization for Standardization, Geneva, Switzerland.

ISO, 2008. Evaluation of measurement data - Guide to the expression of uncertainty in measurement. ISO/IEC Guide 98-3:2008 (JCGM/WG1/100) International Organization for Standardization, Geneva, Switzerland.

ISO, 2014. Hydrometry -- Suspended sediment in streams and canals -- Determination of concentration by surrogate techniques. ISO 11657:2014, International Organization for Standardization, Geneva Switzerland.

ISO, 2017. Guidance for the use of repeatability, reproducibility and trueness estimates in measurement uncertainty estimation, ISO 21748, International Organization for Standardization, Geneva, Switzerland.

Jafar-Sidik, M., Gohin, F., Bowers, D., Howarth, J., Hull, T., 2017. The relationship between Suspended Particulate Matter and turbidity at a mooring station in a coastal environment: consequences for satellite-derived products. Oceanologia 59, 365-378. doi:10.1016/j.oceano.2017.04.003

Jalón-Rojas, I., Schmidt, S., Sottolichio, A., 2015. Turbidity in the fluvial Gironde Estuary (southwest France) based on 10-year continuous monitoring: sensitivity to hydrological conditions. Hydrology and Earth System Science 19, 2805-2819. doi:10.5194/hess-19-2805-2015 
JAMP, 2012. Eutrophication monitoring guidelines: Chlorophyll a in Water. OSPAR Joint Assessment \& Monitoring Programme, Agreement 2012-11.

Jourdin, F., Tessier, C., Le Hir, P., Verney, R., Lunven, M., Loyer, S., Lusven, A., Filipot, J.-F., Lepesqueur, J., 2014. Dual-frequency ADCPs measuring turbidity. Geo-Marine Letters 34, 381-397. doi:10.1007/s00367-014-0366-2

Kappenberg, J., Berendt, M., Ohle, N., Riethmüller, R., Schuster, D., Strotmann, T., 2018. Variation of hydrodynamics and water constituents in the mouth of the Elbe estuary, Germany. Civil Engineering Research Journal 4, 555643. doi:10.19080/CERJ.2018.04.555643

Kerr, A., Cowling, M.J., Beveridge, C.M., Smith, M.J., Parr, A.C.S., Head, R.M., Davenport, J., Hodgkiess, T., 1998. The early stages of marine biofouling and its effects on two types of optical sensors. Environment International 24, 331-343.

Kineke, G.C, Sternberg, R.W., 1992. Measurements of high concentration suspended sediments using the optical backscatterance sensor. Marine Geology, 108, 253-258

Klein, H., 2003. Investigating sediment re-mobilisation due to wave action by means of ADCP echo intensity data Field data from the Tromper Wiek, western Baltic Sea. Estuarine, Coastal and Shelf Science 58, 467-474. doi:10.1016/S0272-7714(03)00113-6.

Krivtsov, V., Howarth, M.J., Jones, S.E., Souza, A.J., Jago, C.F., 2008. Monitoring and modelling of the Irish Sea and Liverpool Bay: An overview and an SPM case study. Ecological Modelling 212, 37-52. doi:10.1016/j.ecolmodel.2007.10.038

Lane, A., Riethmüller, R., Herbers, D., Rybaczokv P., Günther, H., Baumert, H., 2000. Observational data sets for model development. Coastal Engineering 41, 125-153.

Lee, B.J., Kim, J., Hur, J., Choi, I.H., Toorman, E., Fettweis, M., Choi, J.W., 2019. Seasonal dynamics of organic matter composition and its effects on suspended sediment flocculation in river water. Water Resources Research. doi:10.1029/2018WR024486

Lurton, X. 2010. An introduction to underwater acoustics. Springer Verlag $2^{\text {nd }}$ Edition, 680pp.

Maerz, J., Hofmeister, R., van der Lee, E.M., Gräwe, U., Riethmüller, R., Wirtz, K.W., 2016. Maximum sinking velocities of suspended particulate matter in a coastal transition zone. Biogeosciences 13, 4863-4876, doi:10.5194/bg-13-4863-2016

Manov, V., Chang, G.C., Dickey, T.D., 2004. Methods for Reducing Biofouling of Moored Optical Sensors. Journal of Atmospheric and Ocean Technology, 21, 958-968.

Many, G., Bourrin, F., Durrieu de Madron, X., Pairaud, I., Gangloff, A., Doxaran, D., Ody, A., Verney, R., Menniti, C., Le Berre, D., Jacquet, M., 2016. Particle assemblage characterization in the Rhone River ROFI. Journal of Marine Systems 157, 39-51. doi:10.1016/j.jmarsys.2015.12.010

McAnally, W.H., Friedrichs, C., Hamilton, D., Hayter, E., Shrestha, P., Rodriguez, H., Sheremet, A., Teeter, A.M., 2007. Management of fluid mud in estuaries, bays, and lakes. I: Present state of understanding on character and behavior. Journal of Hydraulic Engineering 133, 9-22.

Medwin, H., Clay, C.S., 1998. Fundamentals of acoustical oceanography. Academic Press, New York, 712pp.

Merkelbach, L.M., 2006. A model for high-frequency acoustic Doppler current profiler backscatter from suspended sediment in strong currents. Continental Shelf Research 26, 1316-1335. doi:10.1016/j.csr.2006.04.009

Merkelbach, L.M., Ridderinkhof, H., 2006. Estimating suspended sediment concentration using backscatterance from an acoustic Doppler profiling current meter at a site with strong tidal currents. Ocean Dynamics 56, 153168. doi:10.1007/s10236-005-0036-z

Mishchenko, M.I., Travis, L.D., Lacis, A.A., 2002. Scattering, absorption, and emission of light by small particles. Cambridge University Press, Cambridge.

Moody, J.A., Butman, B., Bothner, M.H., 1987. Near-bottom suspended matter concentration on the continental shelf during storms: estimates based on in-situ observations of light transmission and a particle size dependent transmissometer calibration. Continental Shelf Research 7, 609-628.

Mullison, J., 2017. Backscatter estimation using Broadband Acoustic Doppler Current Profilers - updated. ASCE Hydraulic Measurements \& Experimental Methods Conference, Durham, NH (USA). July 9-12.

Muste, M, Kim, D., Burkhardt, A., Brownson, Z., 2006. Near-transducer errors in Acoustic Doppler Current Profiler measurements. World Environmental and Water Resources Congress, Omaha (Nebraska, USA), May 21-25, doi:10.1061/40856(200)164 
Nauw, J.J., Merckelbach, L.M., Ridderinkhof, H., van Aken, H.M., 2014. Long-term ferry-based observations of the suspended sediment fluxes through the Marsdiep inlet using acoustic Doppler current profilers. Journal of Sea Research 87, 17-29. doi:10.1016/j.seares.2013.11.013

Nauw, J., Linke, P., Leifer, I., 2015. Bubble momentum plume as a possible mechanism for an early breakdown of the seasonal stratification in the northern North Sea. Marine and Petroleum Geology 68, 789-805. doi:10.1016/j.marpetgeo.2015.05.003

Nechad, B., Ruddick, K., Neukermans G., 2009. Calibration and validation of a generic multisensor algorithm for mapping of turbidity in coastal waters. Proceedings SPIE "Remote Sensing of the Ocean, Sea Ice, and Large Water Regions", SPIE Vol. 7473, 74730H.

Nechad, B., Dogliotti, A., Ruddick, K., Doxaran, D., 2016. Particulate backscattering retrieval from remotely-sensed turbidity in various coastal and riverine turbid waters. In: Proc. Living Planet Symposium (ed. Ouwehand, L.), ESA-SP Volume 740, ISBN: 978-92-9221-305-3

Neukermans, G., Ruddick, K., Loisel, H., Roose, P., 2012a. Optimization and quality control of suspended particulate matter concentration measurement using turbidity measurements. Limnology and Oceanography Methods 10 , 1011-1023.

Neukermans, G., Loisel, H., Mériaux, X., Astoreca, R., McKee, D., 2012b. In situ variability of mass-specific beam attenuation and backscattering of marine particles with respect to particle size, density, and composition. Limnology and Oceanography 57, 124-144. doi:10.4319/lo.2011.57.1.0124 1

Ocean Optics Web Book, 2018. A collaborative web-based book on optical oceanography http://www.oceanopticsbook.info/

Pait, A.S., Galdo Jr, F.R., Hartwell, S.I., Mason, A.L., Apeti, D.A., Jeffrey, C.F.G., Hoffman, A.M., Pittman, S.J., 2015. An assessment of nutrients, sedimentation, and total suspended solids (TSS) in the St. Thomas East End Reserves (STEER). NOAA Technical Memorandum NOS/NCCOS 184. Silver Spring, MD. 66pp

Palinkas, C.M., Ogston, A.S., Nittrouer, C.A., 2010. Observations of event-scale sedimentary dynamics with an instrumented bottom boundary-layer tripod. Marine Geology 274, 151-164. doi:10.1016/j.margeo.2010.03.012

Pearlman, S.R., Costa, H.S., Jung, R.A., McKeown, J.J., Pearson, H.E., 1995. Solids (section 2540). In: Eaton, A.D., Clesceri, L.S., Greenberg, A.E. (Eds.), Standard Methods for the Examination of Water and Wastewater. American Public Health Association, Washington DC, USA, pp. 2-53-2-64.

Press, W.H., Flannery, B.P., Teukolsky, S.A., Vetterling, W.T., 1989. Numerical recipes. Cambridge University Press, $702 \mathrm{pp}$.

Puleo, J.A., Johnson, R.V., Butt, T., Kooney, T.N., Holland, K.T., 2006. The effect of air bubbles on optical backscatter sensors. Marine Geology 230, 87-97. doi:10.1016/j.margeo.2006.04.008

Rai, A.K., Kumar, A., 2015. Continuous measurement of suspended sediment concentration: Technological advancement and future outlook. Measurements 76, 209-227. doi:10.1016/j.measurement.2015.08.013

Ramsey, M.H., Ellison, S.L.R. (Eds.). 2007. Measurement uncertainty arising from sampling: A guide to methods and approaches. Eurachem/EUROLAB/ CITAC/Nordtest/AMC Guide.

Randolph, K., Dierssen, H.M., Twardowski, M., Cifuentes-Lorenzen, A., Zappa, C.J., 2014. Optical measurements of small deeply penetrating bubble populations generated by breaking waves in the Southern Ocean. Journal of Geophysical Research Oceans 119, 757-776. doi:10.1002/2013JC009227.

Rasmussen, P.P., Gray, J.R., Glysson, G.D., Ziegler, A.C., 2009. Guidelines and procedures for computing time-series suspended-sediment concentrations and loads from in-stream turbidity-sensor and streamflow data: U.S. Geological Survey Techniques and Methods Book 3, chap. C4, 53 pp.

Ridd, P., Larcombe, P., 1994. Biofouling control for optical backscatter suspended sediment sensors. Marine Geology, 116, 255-258.

Röttgers, R., Heymann K., Krasemann, H., 2014. Suspended matter concentrations in coastal waters: Methodological improvements to quantify individual measurement uncertainty. Estuarine, Coastal and Shelf Science 151, 148-155. doi:10.1016/j.ecss.2014.10.010

Rouhnia, M., Keyvani, A., Strom, K., 2014. Do changes in the size of mud flocs affect the acoustic backscatter values recorded by a Vector ADV? Continental Shelf Research 84, 84-92. doi:10.1016/j.csr.2014.05.015

Rymszewicz, A., O'Sullivan, J.J., Bruen, M., Turner, J.N., Lawler, D.M., Conroy, E., Kelly-Quinn, M., 2017. Measurement differences between turbidity instruments, and their implications for suspended sediment 
concentration and load calculations: A sensor inter-comparison study. Journal of Environmental Management 199, 99-108. doi:10.1016/j.jenvman.2017.05.017

Sadar, M., 1999. Turbidimeter instrument comparison: Low-level sample measurements technical information series. Hach Company, report D90.5, 55pp.

Sahin, C., Verney, R., Sheremet, A., Voulgaris, G., 2017. Acoustic backscatter by suspended cohesive sediments: field observations, Seine Estuary, France. Continental Shelf Research 134, 39-51. doi:10.1016/j.csr.2017.01.003

Salehi, M., Strom, K., 2011. Using velocimeter signal to noise ratio as a surrogate measure of suspended mud concentration. Continental Shelf Research 31, 1020-1032. doi:10.1016/j.csr.2011.03.008

Schmechtig, C., Thierry, V., the Bio Argo Team, 2015. Argo quality control manual for biogeochemical data. doi:10.13155/40879

Schwarz, C., Cox, T.J.S., Van Engeland, T., Van Oevelen, D., Van Belzen, J, van de Koppel, J, Soetaert, K., Bouma, T.J., Meire, P, Temmerman, S., 2017. Field estimates of floc dynamics and settling velocities in a tidal creek with significant along-channel gradients in velocity and SPM. Estuarine, Coastal and Shelf Science 197, 221-235. doi:10.1016/j.ecss.2017.08.041

Schwendeman, M., Thomson, J., 2015. Observations of whitecap coverage and the relation to wind stress, wave slope, and turbulent dissipation, Journal of Geophysical Research Oceans 120, 8346-8363. doi:10.1002/2015JC011196.

Sheng, J., Hay, A.E., 1988. An examination of the spherical scatterer approximation in aqueous suspensions of sand. Journal of the Acoustical Society of America 83, 598-610.

Shreve, E.A., Downs, A.C., 2005. Quality-assurance plan for the analysis of fluvial sediment. U.S. Geological Survey Kentucky Water Science Center Sediment Laboratory, USGS Open-File Report 2005-1230, 28pp.

Slade, W.H. Boss, E., Russo, C. 2011. Effects of particle aggregation and disaggregation on their inherent optical properties. Optics Express 19, 7945-7959. doi:10.1364/OE.19.007945

Slade, W.H., Agrawal, Y.C., Mikkelsen, O.A., 2013. Comparison of measured and theoretical scattering and polarization properties of narrow size range irregular sediment particles. Presented at Oceans, San Diego, $23-7$.

Sottolichio, A., Hurther, D., Gratiot, N., Bretel, P., 2011. Acoustic turbulence measurements of near-bed suspended sediment dynamics in highly turbid waters of a macrotidal estuary. Continental Shelf Research, 31, S36-S49. doi:10.1016/j.csr.2011.03.016

Spinrad, R.W., Yentsch C.M., Brown, J., Dortch, Q., Haugen, E., Revelante, N., Shapiro, L., 1989. The response of beam attenuation to heterotrophic growth in a natural population of plankton. Limnology and Oceanography 34, 1601-1605.

Stanton, T.K., 1989. Simple approximate formulas for backscattering of sound by spherical and elongated objects. Journal of the Acoustical Society of America 86, 1499-1510.

Stavn, R.H., Rick, H.J., Falster, A.V., 2009. Correcting the errors from variable sea salt retention and water of hydration in loss on ignition analysis: Implications for studies of estuarine and coastal waters. Estuarine Coastal and Shelf Science 81, 575-582. doi:10.1016/j.ecss.2008.12.017

Strickland, J.D.H., Parsons, T.R., 1968. A practical handbook of seawater analysis. Bulletin 167. Fisheries Research Board of Canada, Ottawa, Canada, 181-184.

Sutherland, T.F., Lane, P.M., Amos, C.L., Downing, J., 2000. The calibration of optical backscatter sensors for suspended sediment of varying darkness levels. Marine Geology, 162, 587-597. doi:10.1016/S00253227(99)00080-8

Tessier, C., 2006. Charactérisation et dynamique des turbidités en zone côtière: l'exemple de la région marine Bretagne Sud. PhD thesis Université de Bordeaux, France, 400pp.

Tessier, C., Le Hir, P., Lurton, X., Castaing, P., 2008. Estimation de la matière en suspension à partir de l'intensité acoustique rétrodiffusée des courantomètres acoustiques à effet Doppler (ADCP). Comptes Rendus Geoscience 340, 57-67.

Thorne, P.D., Vincent, C.E., Hardcastle, P.J., Rehman, S., Pearson, N., 1991. Measuring suspended sediment concentrations using acoustic backscatter devices. Marine Geology, 98, 7-16.

Thorne, P.D., Hardcastle, P.J., Holdaway, G.P., Born, A.J., 1994. Analysis of results obtained from a triple frequency acoustic backscatter system for measuring suspended sediments, Proceedings of the 6th International Conference on Electronic Engineering in Oceanography, 394, 83-89.

Thorne, P.D., Hanes, D.M., 2002. A review of acoustic measurement of small-scale sediment processes. Continental Shelf Research 22, 603-632. doi:10.1016/S0278-4343(01)00101-7 
Thorne, P.D., MacDonald, I.T., Vincent C.E., 2014. Modelling acoustic scattering by suspended flocculating sediments. Continental Shelf Research 88, 81-91. doi:10.1016/j.csr.2014.07.003

TMAP, 2009. Monitoring Handbook: Eutrophication - Nutrients (version 16.09.2009, TMAG 09-2). http://www.waddensea-secretariat.org/monitoring-tmap/manual-guidelines

Topping, D.J., Wright, S.A., 2016, Long-term continuous acoustical suspended-sediment measurements in riversTheory, application, bias, and error. U.S. Geological Survey, USGS Professional Paper 1823, 98 pp. doi:10.3133/pp1823.

Tzeng, M.W., Dzwonkowski, B., Park, K., 2016. Data processing for a small-scale long-term coastal ocean observing system near Mobile Bay, Alabama. Earth and Space Science 3, 510-522. doi:10.1002/2016EA000188.

van der Hout, C.M., Gerkema, T., Nauw, J.J., Ridderinkhof, H., 2015. Observations of a narrow zone of high suspended particulate matter (SPM) concentrations along the Dutch coast. Continental Shelf Research 95, 2738. doi:10.1016/j.csr.2015.01.002

Van der Hout, C.M., Witbaard, R., Bergman, M.J.N., Duineveld, G.C.A., Rozemeijer, M.J.C., Gerkema, T., 2017. The dynamics of suspended particulate matter (SPM) and chlorophyll-a from intratidal to annual time scales in a coastal turbidity maximum. Journal of Sea Research 127, 105-118. doi:10.1016/j.seares.2017.04.011

Wagner, R.J., Boulger Jr, R.W., Oblinger, C.J., Smith, B.A., 2006. Guidelines and standard procedures for continuous water-quality monitors - Station operation, record computation, and data reporting. U.S. Geological Survey, USGS Techniques and Methods 1-D3, 51 pp. http://pubs.water.usgs.gov/tm1d3

Waldmann, C., Tamburri, M., Prien, R.D., Fietzek, P., 2010. Assessment of sensor performance. Ocean Science 6, 235-245. doi:10.5194/os-6-235-2010

Wang, D.W., Wijesekera, H.W., Teague, W.J., Rogers, W.E., Jarosz, E., 2011. Bubble cloud depth under a hurricane. Geophysical Research Letters 38, L14604. doi:10.1029/2011GL047966

Wang, D.W., Wijesekera, H.W., Jarosz, E., Teague, W.J., 2016. Turbulent diffusivity under high winds from acoustic measurements of bubbles. Journal of Physical Oceanography 46, 1593-1613. doi:10.1175/JPO-D-15-0164.1

Whelan, A., Regan, F., 2006. Antifouling strategies for marine and riverine sensors. Journal of Environmental Monitoring 8, 880-886. doi:10.1039/b603289c

Wilcox, R.R., 2001, Fundamentals of modern statistical methods substantially improving power and accuracy. Springer-Verlag, 249pp.

Woźniak, S.B., Meler, J., Lednicka, B., Zdun, A., Stoń-Egiert, J. 2011. Inherent optical properties of suspended particulate matter in the southern Baltic Sea. Oceanologia 53, 691-729. doi:10.5697/oc.53-3.691

Yeats, P.A., Brügmann, L., 1990. Suspended particulate matter: collection methods for gravimetric and trace metal analysis. ICES Techniques in Marine Environmental Sciences 7, 9pp.

Yentsch, C.S., 1962. Measurement of visible light absorption by particulate matter in the ocean. Limnology and Oceanography 7, 207-217.

Zhang, X., Stavn, R.H., Falster, A.U., Gray, D., Gould Jr, R.W., 2014. New insight into particulate mineral and organic matter in coastal ocean waters through optical inversion. Estuarine, Coastal and Shelf Science 149, 1-12. doi:10.1016/j.ecss.2014.06.003

Ziegler, A.C., 2003. Breakout session 1 - Definition of optical methods for turbidity and data reporting. In: Proceedings of the Federal Interagency Workshop on Turbidity and Other Sediment Surrogates (Gray, J.R., Glysson, G.D., eds.), U.S. Geological Survey, USGS Circular 1250, 9 - 13.

http://water.usgs.gov/pubs/circ/2003/circ1250/ 
Version: August 11, 2019;

Table 1: Bulk mineralogical composition (in \%) of the SPM in the English Channel and southern North Sea sampled with a centrifuge during transect or anchoring of the vessel. Offshore Somme mouth, Dover Strait, Calais, Zeebrugge and north of the Rhine mouth. Qtz=Quartz; Kspar =K-feldspar; Plag=Plagioclase; Carb=Sum of Calcite, Mg-rich Calcite, Dolomite, Aragonite; $\mathrm{Am}=$ Amorphous fraction (organic matter and biogenic opal); Hal=Halite; NClays=Sum of nonclay minerals; Kaol=Kaolinite; $\mathrm{Chl}=$ Chlorite; $2: 1=$ Sum of 2:1 clays and micas; Clays= Sum of Kaol+chl+2:1. The samples have been collected with a centrifuge (water intake about $4 \mathrm{~m}$ below surface) and analysed with XRD (data from Adriaens et al., 2018).

\begin{tabular}{|l|c|c|c|c|c|c|c|c|c|c|c|}
\hline & Qtz & Kspar & Plag & Carb & Am & Hal & NClays & Kaol & Chl & $2: 1$ & Clays \\
\hline Somme & 29 & 2 & 3 & 30 & 15 & 5 & $\mathbf{8 6}$ & $<1$ & $<1$ & 10 & $\mathbf{1 0}$ \\
\hline Dover Strait & 22 & 3 & 2 & 21 & 18 & 7 & $\mathbf{8 4}$ & 2 & 4 & 20 & $\mathbf{2 6}$ \\
\hline Calais & 10 & 1 & $<1$ & 29 & 22 & 3 & $\mathbf{6 6}$ & $<1$ & 2 & 31 & $\mathbf{3 3}$ \\
\hline Zeebrugge & 20 & 1 & 5 & 24 & 10 & 2 & $\mathbf{6 1}$ & 2 & 4 & 33 & $\mathbf{3 9}$ \\
\hline Rhine offshore & 29 & 4 & 1 & 21 & 12 & 3 & $\mathbf{7 0}$ & 1 & 2 & 27 & $\mathbf{3 0}$ \\
\hline Rhine nearshore & 17 & 4 & 2 & 21 & 16 & 1 & $\mathbf{6 1}$ & 2 & 1 & 35 & $\mathbf{3 8}$ \\
\hline
\end{tabular}


Version: August 11, 2019;

Table 2: List of additional state parameter and their measuring methods that are necessary to interpret the results of long-term SPMC measurements with more accuracy and completeness (sensor: instrument that can be used in long-term measurements; water/centrifuge sample: can be measured occasionally; station: nearby meteorological, tidal, or wave station; Lol=loss on ignition, $\mathrm{POC}=$ particulate organic carbon, $\mathrm{PON}$ : particulate organic nitrate, XRD: $\mathrm{x}$-ray diffractometer).

\begin{tabular}{|c|c|c|}
\hline State parameter & Method & Type \\
\hline Particle size in situ & $\begin{array}{l}\text { laser diffractometer, holography, optical } \\
\text { camera systems }\end{array}$ & sensor \\
\hline Particle size in lab & Grain size analysis (primary particle size) & $\begin{array}{l}\text { water sample, } \\
\text { centrifuge sample }\end{array}$ \\
\hline Particle composition & $\begin{array}{l}\text { LOI: Organic matter } \\
\text { Element analysis: POC, PON } \\
\text { XRD: Mineral composition } \\
\text { Pigment analysis: Chlorophyll } \\
\text { Colorimetric analysis: TEP concentration } \\
\text { Electron microscopy }\end{array}$ & $\begin{array}{l}\text { water sample, } \\
\text { centrifuge sample }\end{array}$ \\
\hline Particle composition & $\begin{array}{l}\text { Fluorimeter: Fluorescence } \\
\text { Primary production: Fast Repetition Rate } \\
\text { Fluorimeter }\end{array}$ & sensor \\
\hline \multirow{2}{*}{ Particle shape } & Holography, floc camera & sensor \\
\hline & Electron microscopy & water sample \\
\hline Turbulence & High frequency Acoustic Doppler Sensor & sensor \\
\hline Currents & Acoustical Doppler Sensor & sensor \\
\hline Waves & Acoustic Doppler Profiler, Wave rider, floater & sensor or station \\
\hline Density & Conductivity, Temperature & sensor \\
\hline Water level & Pressure sensor; tide gauge & sensor, station \\
\hline Distance to bed & Acoustic altimeter & sensor \\
\hline Meteorology & from relevant meteorological stations & station \\
\hline
\end{tabular}


Table 3: Quantification and nature (random or bias) of uncertainties (in \% based on normalized RMSE) from the examples described in chapter 3. Negative (positive) bias corresponds to an underestimation (overestimation) of the actual value.

\begin{tabular}{|c|c|c|c|c|c|c|c|}
\hline \multirow{2}{*}{ Source of uncertainty } & \multirow{2}{*}{ Detectable } & \multirow{2}{*}{ Correctable } & \multicolumn{2}{|c|}{ Uncertainty without correction } & \multicolumn{2}{|c|}{ Uncertainty with correction } & \multirow[t]{2}{*}{ Comment } \\
\hline & & & random & bias & random & bias & \\
\hline \multicolumn{8}{|l|}{ 3.1 Sensor } \\
\hline 3.1.1 Factory calibration & yes & yes & - & $\pm 15 \%$ & - & less than $\pm 2 \%$ & $\begin{array}{l}\text { not fully conclusive, as sensors } \\
\text { were checked after intense usage }\end{array}$ \\
\hline 3.1.1 Optical degradation & yes & yes & - & $20 \%$ & - & $\pm 5 \%$ & Figure 1 \\
\hline 3.1.1 Voltage supply & yes & yes & & $-90 \%$ & & $\pm 10 \%$ & Figure 2 \\
\hline 3.1.2.Sensitivity of sensor & yes & no & $<25 \%$ & & no example & no example & depends on measuring range \\
\hline 3.1.3 Inter-sensor variability & yes & no & $2 \%$ & $\begin{array}{l}\text { no example } \\
\text { provided }\end{array}$ & $\begin{array}{l}\text { no example } \\
\text { provided }\end{array}$ & $\begin{array}{l}\text { no example } \\
\text { provided }\end{array}$ & $\begin{array}{l}\text { Figure } 3 \\
\text { Comparing sensors of same type } \\
\text { and applying the same set of } \\
\text { sensor output to sample SPMC } \\
\text { calibration constants for all sensors }\end{array}$ \\
\hline \multicolumn{8}{|l|}{ 3.2 Environment } \\
\hline 3.2.1 Biofouling & yes & no $^{1}$, yes $^{2}$ & - & $\begin{array}{c}\text { positive, large (up } \\
\text { to }>100 \%)^{1} \\
\text { negative }^{2} \\
\end{array}$ & $f(\text { no of beams })^{2}$ & $\begin{array}{l}\text { data loss }{ }^{1} \\
\text { partial data loss }\end{array}$ & $\begin{array}{l}{ }^{1} \text { optical sensors } \\
\text { 2acoustical sensors with }>3 \text { beams }\end{array}$ \\
\hline 3.2.2 Saturation & yes & no & & Negative $^{1}$ & - & - & ${ }^{1}$ numerically not specified \\
\hline 3.2.2 Ambiguity & yes & yes & not specified & - & $-\quad-\quad$ & - & \\
\hline 3.2.3 Air bubbles & yes & no & - & - & $\sqrt{2}$ & ${ }^{1}$ data loss & ${ }^{1}$ optical and acoustical sensors \\
\hline 3.2.4 Density stratification & yes & yes & & less than $\pm 43 \%$ & 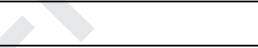 & - & acoustical sensors \\
\hline 3.3 Sample & & & & +2 & & & \\
\hline $\begin{array}{l}\text { one filter } \\
\text { triplicate filter } \\
\text { volume method }\end{array}$ & $\begin{array}{l}\text { yes }^{1} \\
\text { yes }^{1} \\
\text { yes }^{2}\end{array}$ & no & $\begin{array}{c}2 \%-10 \% \\
1 \%-6 \% \\
3 \%\end{array}$ & $1 \%-50 \%^{3}$ & $\begin{array}{l}- \\
- \\
-\end{array}$ & $\begin{array}{c}\text { not correctable } \\
\text { not correctable } \\
<5 \%^{3}\end{array}$ & $\begin{array}{l}{ }^{1} \text { only random uncertainty } \\
2 \text { random + bias } \\
{ }^{3} \text { random bias per sample ; } \\
\text { depends on SPMC (>50-<1 mg/l) }\end{array}$ \\
\hline 3.4 Model & & & & $\mathrm{r}$ & & & \\
\hline 3.4.2 Regression model & yes & no & $10 \%-60 \%^{1}$ & $0->30 \%^{1,2}$ & & & $\begin{array}{l}{ }^{1} \text { depends on } \mathrm{R}^{2} \text { and } \mathrm{T}_{\mathrm{z} \text {-std }} \\
{ }^{2} \text { depends on regression model }\end{array}$ \\
\hline $\begin{array}{l}\text { 3.4.3 Inherent particle } \\
\text { properties }\end{array}$ & yes $^{1}$ & partly & & $\begin{array}{c} \pm 50 \%^{2} \\
40 \%-60 \%^{3} \\
50 \%-200 \%^{4} \\
\pm 10 \%^{5}\end{array}$ & & & $\begin{array}{l}{ }^{1} \text { only with reference SPMC } \\
{ }^{2} \text { geographical variation, Figure } 15 \\
{ }^{3} \text { organic content, Figure } 16 \\
{ }^{4} \text { sand in suspension, Figure } 17+18 \\
{ }^{5} \text { in lab vs in situ calibration }\end{array}$ \\
\hline $\begin{array}{l}\text { 3.4.4 Optic vs. acoustic } \\
\text { sensors }\end{array}$ & yes & no & $50-100 \%^{2}$ & $\begin{array}{c}40 \%^{1} \\
O(100 \%)^{2}\end{array}$ & & & $\begin{array}{l}\text { 1acoustic sensor calibrated with } \\
\text { sample SPMC, Figure } 19 \\
\text { 2acoustic sensor calibrated with } \\
\text { OBS, Figure 19, } 20\end{array}$ \\
\hline
\end{tabular}


Version: August 11, 2019;

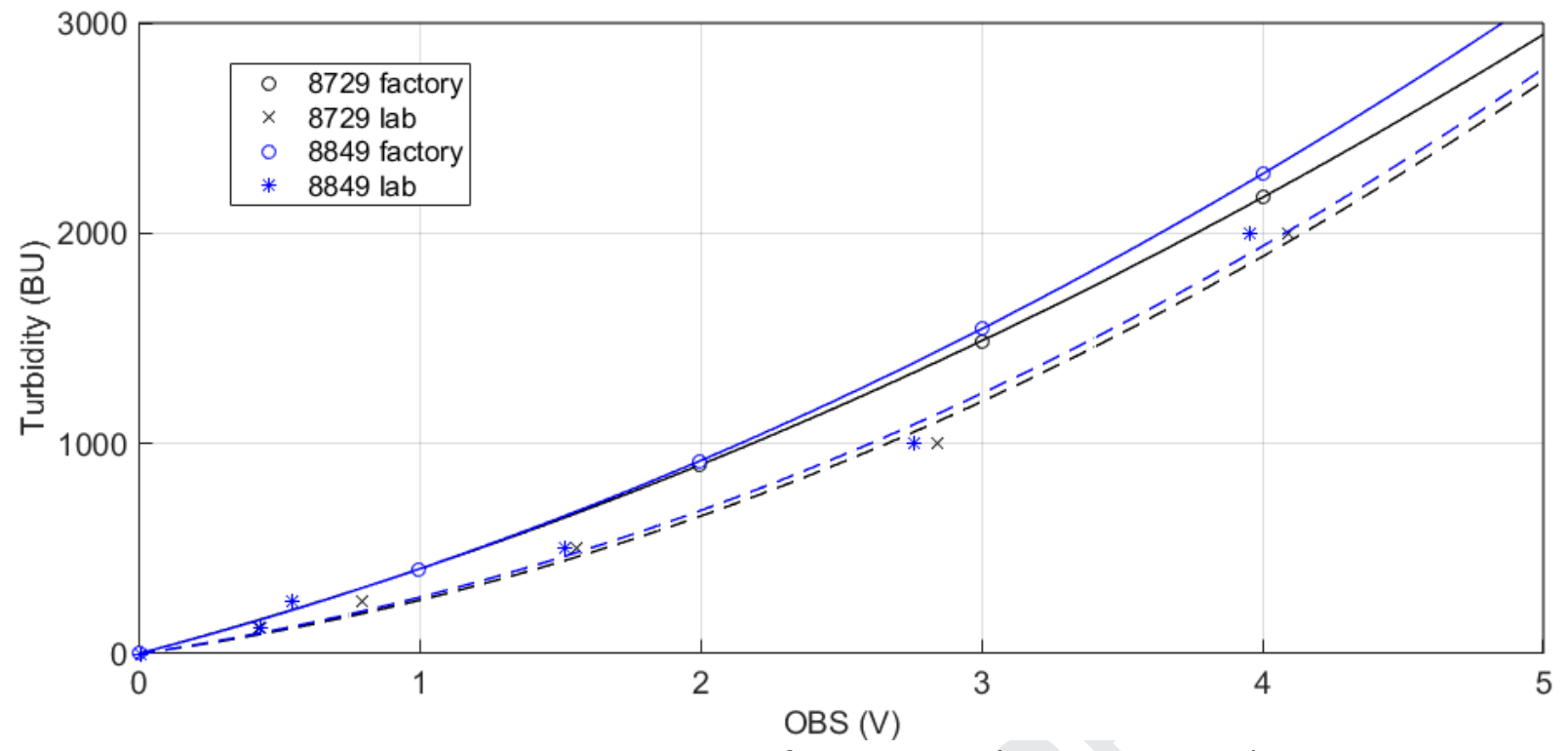

Figure 1: Factory and in lab sensor calibration of two OBS3+ (8729 and 8849). The laboratory calibration was done after 421 (8729) and 329 (8849) days of measurements in high turbid (SPMC > 1g/l) sea water during the period 2014-2016. The solid lines are the $2^{\text {nd }}$ order polynomial fittings provided by the factory, the dashed lines are the $2^{\text {nd }}$ order fittings after recalibration. 
Version: August 11, 2019;
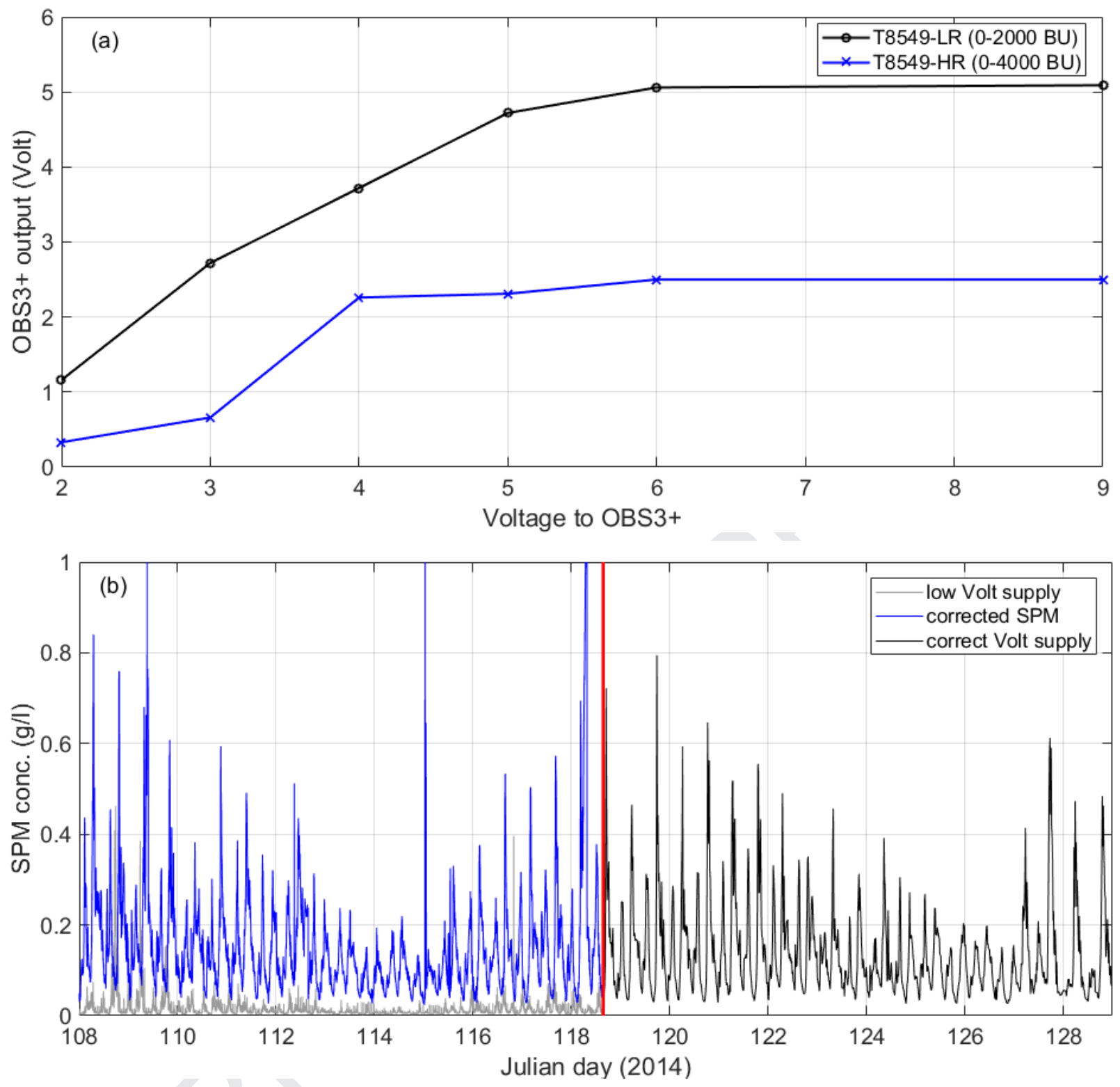

Figure 2: (a) Power provided by an external source to a OBS3+ (T8549) measuring in high and low range in a standard solution of 2000 AMCO Clear ${ }^{\circledR}$ equivalent BU. When the voltage drops below $6 \mathrm{~V}$, the sensor registers low voltage output values. (b) SPMC time series from the Belgian nearshore measured with a OBS3+ that was attached to a SonTek Hydra data logging system for storage and battery supply. The system was replaced on day 118.7 by a similar system. The first system was not providing the minimum of $6 \mathrm{~V}$ to the $\mathrm{OBS}$, the second system worked correctly. In blue is shown the corrected time series. 
Version: August 11, 2019;

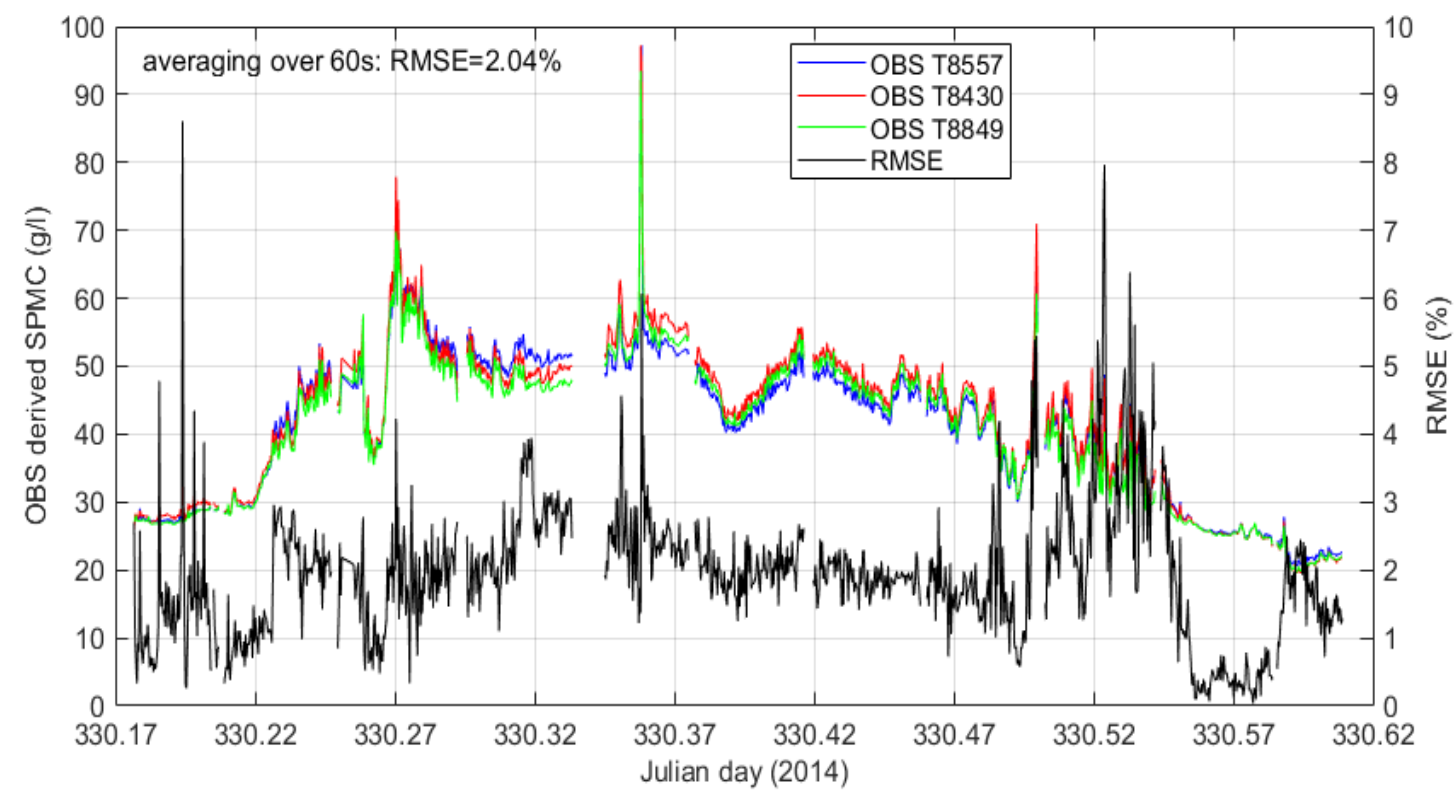

Figure 3: Inter-sensor variability between three $O B S 3+$ sensors measuring at about $50 \mathrm{~cm}$ from each other during a tidal cycle (Belgian nearshore area). The sensors have been calibrated using the same sample SPMC. The differences between the OBS's are not constant but change during the course of the tide. This points possibly to variations in the SPMC (sensor are located about $50 \mathrm{~cm}$ from each other) or to different sensitivities of the individual sensors. 
Version: August 11, 2019;

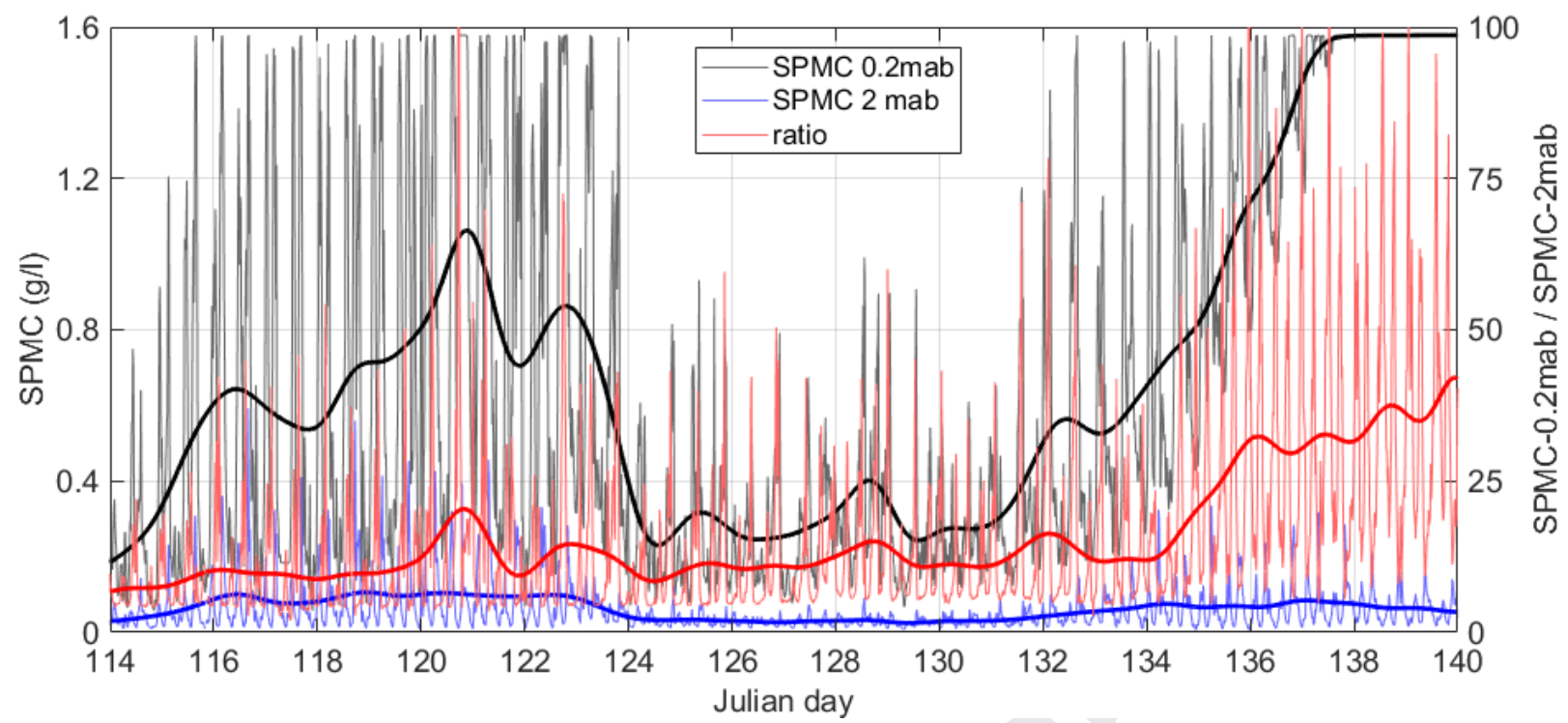

Figure 4: The time series collected in the Belgian nearshore area show the OBS derived SPMC at 0.2 and 2 mab (left axis) and the ratio between SPMC at 0.2 and at 2 mab (right axis). The thick lines are the low-passed filtered data. The OBS at 0.2 mab was covered by barnacles, while the one at 2 mab was not affected by biofouling. The low-pass filtered ratio indicates that biofouling started to affect the OBS at 0.2 mab from about day 134 onward. The OBS at 0.2 mab saturated during the measuring period when the SPMC exceeded about $1.6 \mathrm{~g} / \mathrm{l}$. 
Version: August 11, 2019;

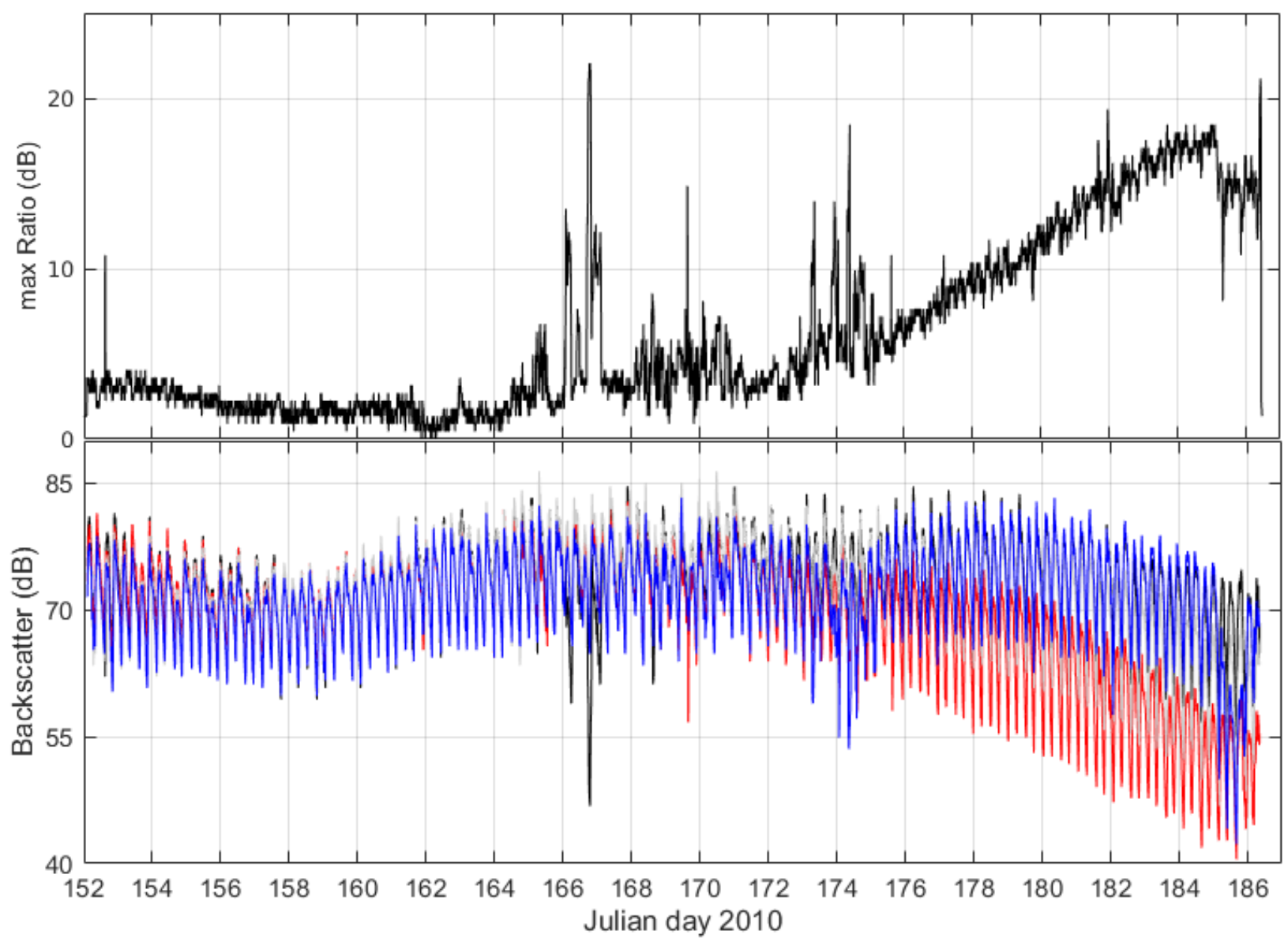

Figure 5: 35-day time series of cross-beam backscatter ratio (up) and the acoustic backscatter intensity (down) recorded by an upward-looking 1.2 $\mathrm{MHz} R D I A D C P$ ( $5^{\text {th }}$ bin at about $1.5 \mathrm{~m}$ above the ADCP is shown) moored offshore the Belgian coast. Changes in the cross beam backscatter ratio, $R_{v}$, occurred from day 165 onward and from day 172 a gradual increase is observed. Superposed are quarter-diurnal variations in $R_{v}$ of the order of 2-3 $d B$ that result from the deposition of fine sediments during slack water and the subsequent erosion during increasing currents. The recovered ADCP was massively fouled by barnacles, tube building worms and hydrozoa. 


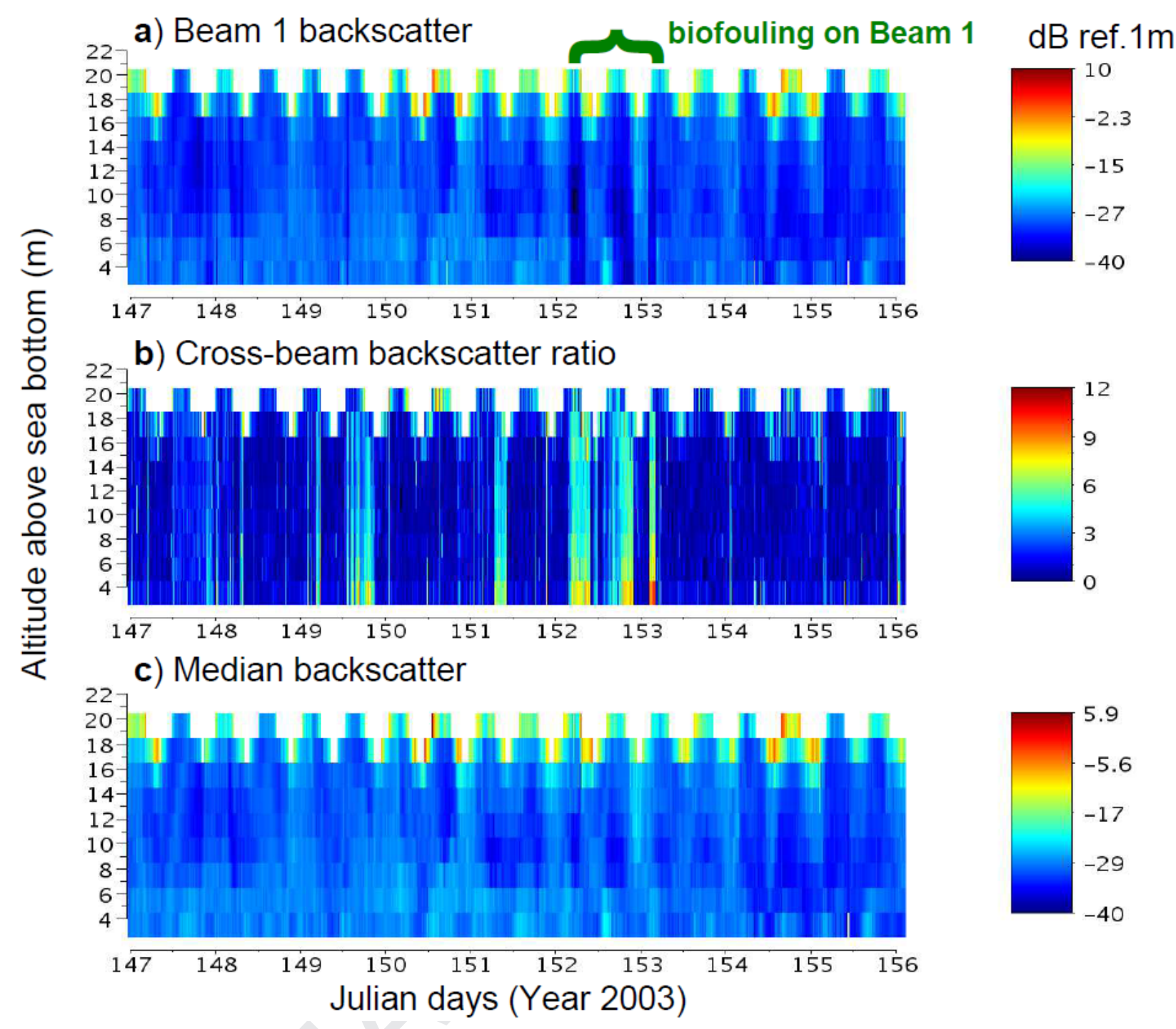

Figure 6: 9-day time series of the backscattering strength vertical profile recorded by an upward-looking $500 \mathrm{kHz}$ ADCP (Nortek ADP) moored on the sea bottom at $20 \mathrm{~m}$ depth in the Bay of Vilaine, South of Brittany (France). The surface oscillation corresponds to the tidal elevation. a) Volume backscatter strength measured by the transducer 1. At day 152 a biofouling event caused a sudden drop in all backscatter values of the profile, by nearly $10 \mathrm{~dB}$. Following, backscatter values rise again and drop again until day 153. b) Cross-beam backscatter ratio $R_{v}$. Biofouling events on other transducers are identified at days 149 and 151 . c) Median acoustic backscatter intensity. The median allows a computation of a backscattering strength without perturbations by the biofouling. 

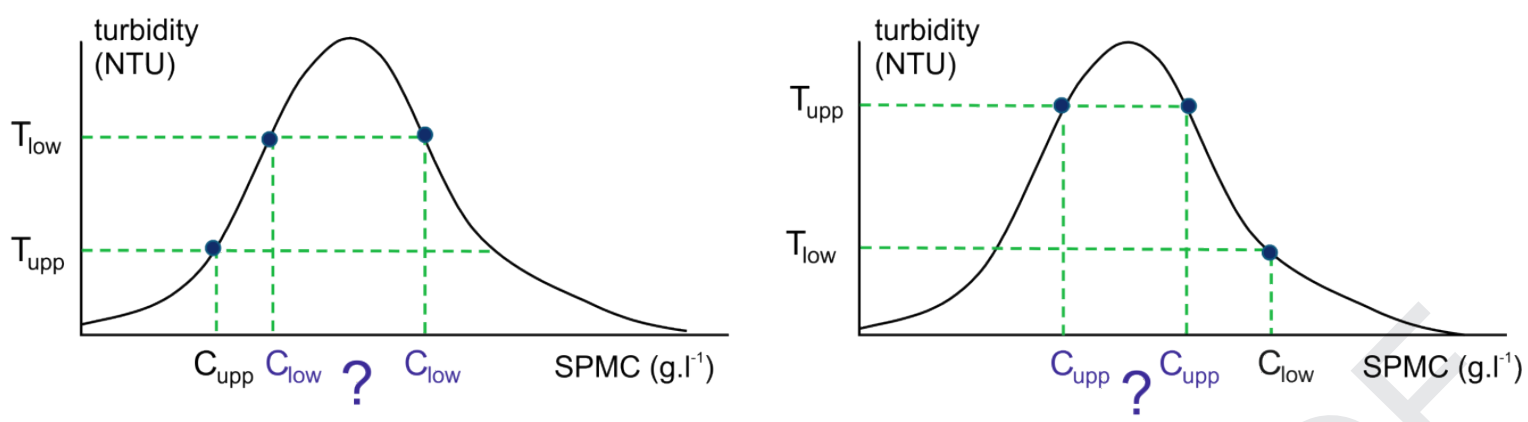

Figure 7: Typical bell-shaped backscatters curve of an OBS (Sottolichio et al., 2011), meaning that a given OBS output can correspond to two SPMC values. The figure shows how this ambiguity can be solved by using two superimposed sensors. Left: when the upper probe records lower turbidity than the lower probe, upper concentration is solved, but the lower remains unsolved. Right: when the upper probe records higher turbidity than the lower one, lower concentration is solved, but the higher remains ambiguous and unsolved. 

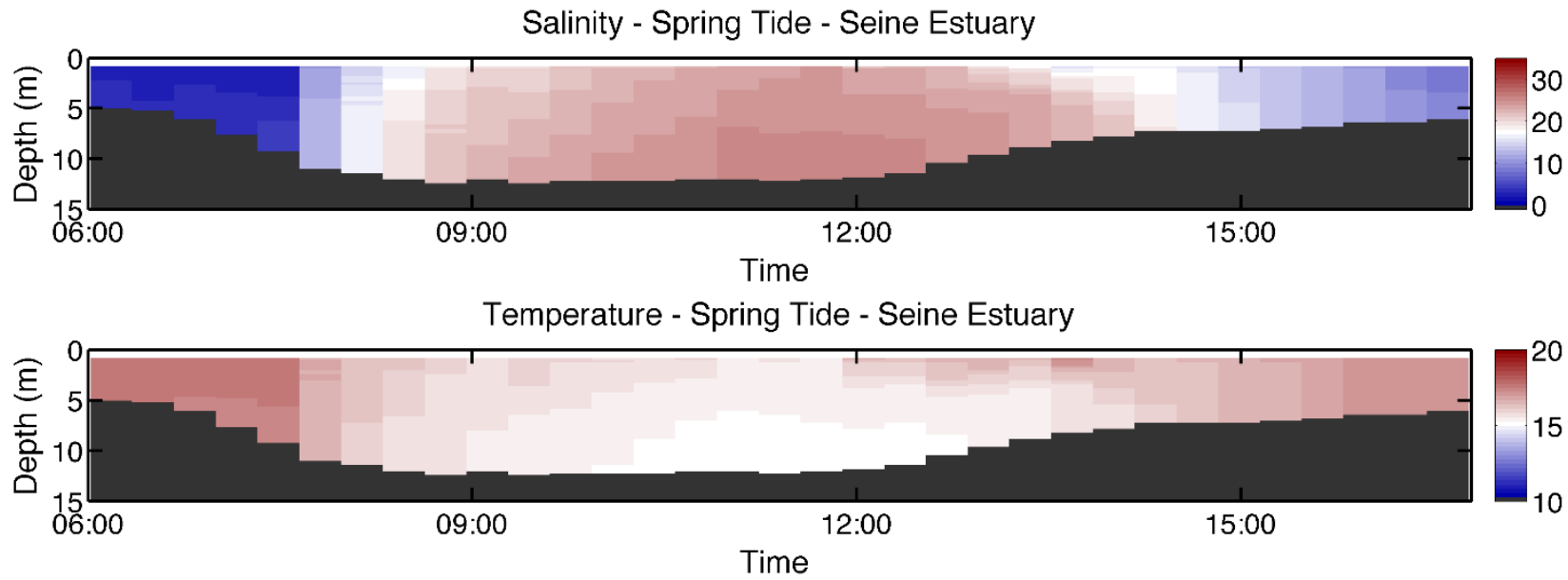

Water attenuation difference : variable salinity/temperature - fixed salinity/temperature

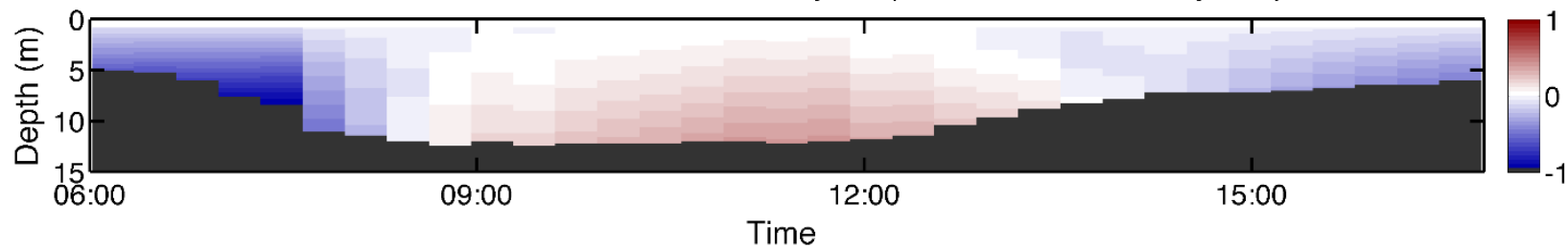

Figure 8: Salinity (top panel) and temperature $\left({ }^{\circ} \mathrm{C}\right.$, middle panel) variability in the Seine Estuary turbidity maximum zone during spring tide. The lower panel shows the water attenuation correction differences (in $\mathrm{dB}$ ) with and without consideration of salinity and temperature variability. 
Version: August 11, 2019;
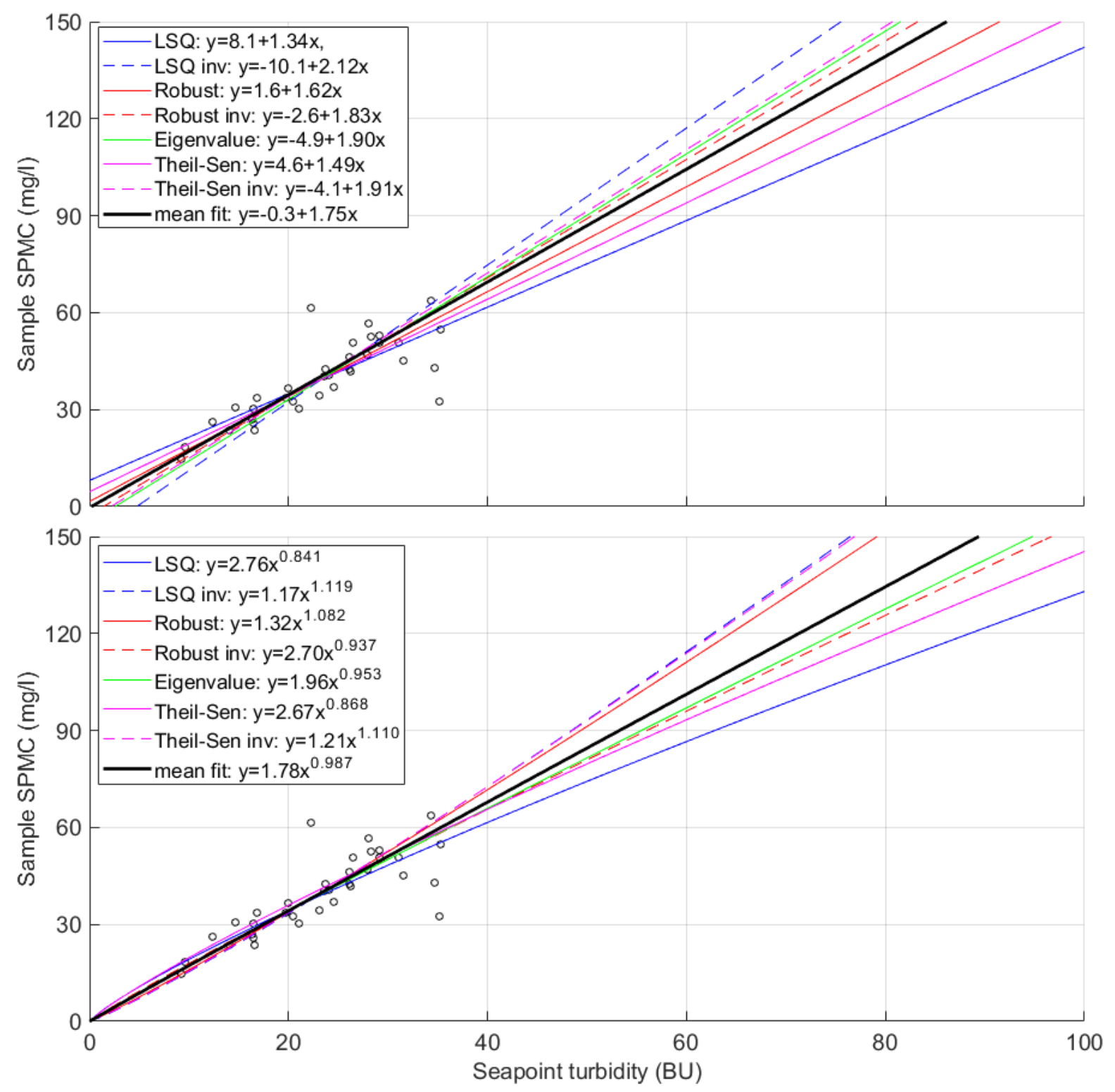

Figure 9: Relating sample SPMC to sensor output using different linear regression models for the actual and the log transformed data $\left(R^{2}=0.61,26\right.$ samples). The data have been collected in the Belgian nearshore area and consist of 26 sample SPMC and corresponding turbidity values from a Seapoint OBS. The solid lines correspond to the relationship $B U=f($ sample SPMC) and the dash lines to sample $S P M C=f(B U)$. The bold line is the mean of the 7 regressions and consist of $a$ baseline to show the variability of the different regression models (compare sections 3.4 .2 and 4.1.4). 
Version: August 11, 2019;

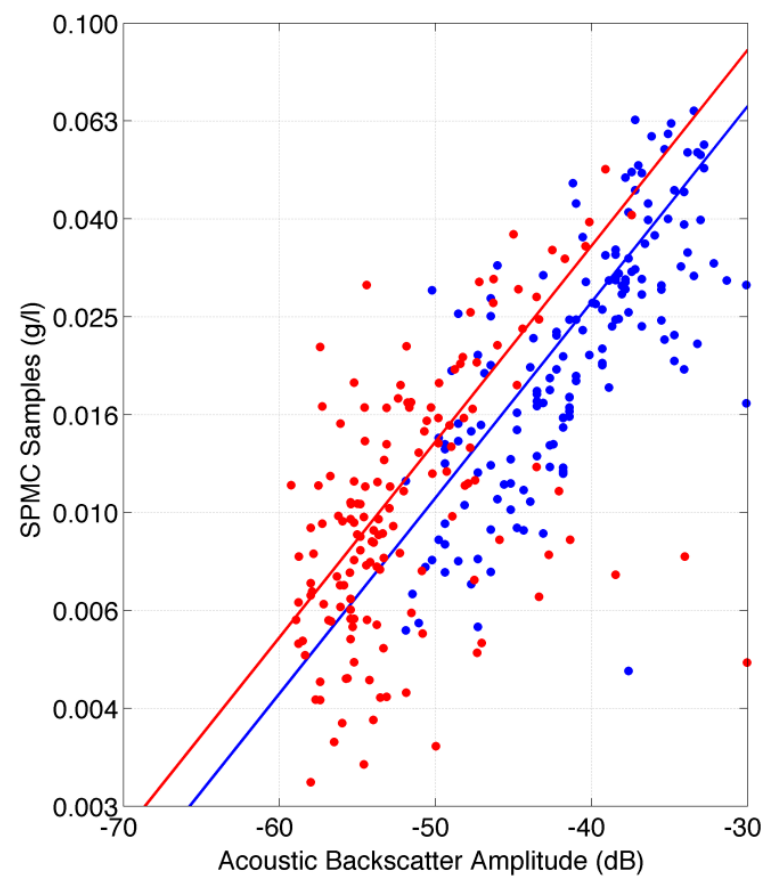

Figure 10: Direct calibration of a profiling acoustic sensor (Nortek AWAC) against sample SPMC in a moderately turbid environment (<100 mg/l) located at the mouth of the Seine estuary (red dots: surface samples, blue dots: bottom samples). 
Version: August 11, 2019;

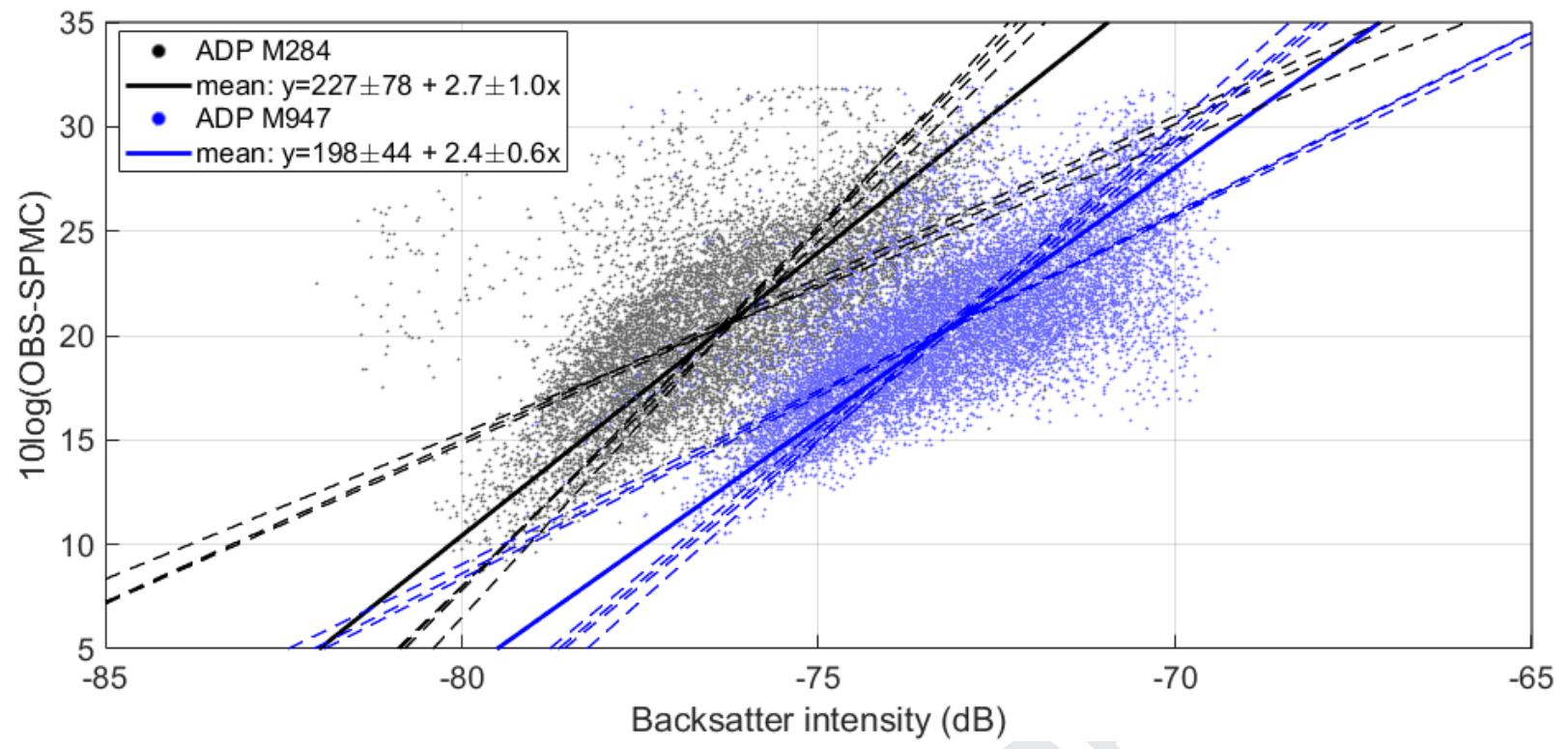

Figure 11: Relating OBS derived SPMC to the backscatter intensity of the first bin of two Sontek $3 \mathrm{MHz}$ ADP profilers (M284, M947) using linear regression models. The data set consists of about one-year data collected in 2013 in the Belgian nearshore area; every sensor measured about half of the period (M284: $\left.R 2=0.4 ; M 947: R^{2}=0.6\right)$. The dashed lines represent the regressions of 7 different models (see text) and the bold lines show the mean over the regressions, which is our baseline to show the variability of the different regression models (compare sections 3.4.2 and 4.1.4). 
Version: August 11, 2019;
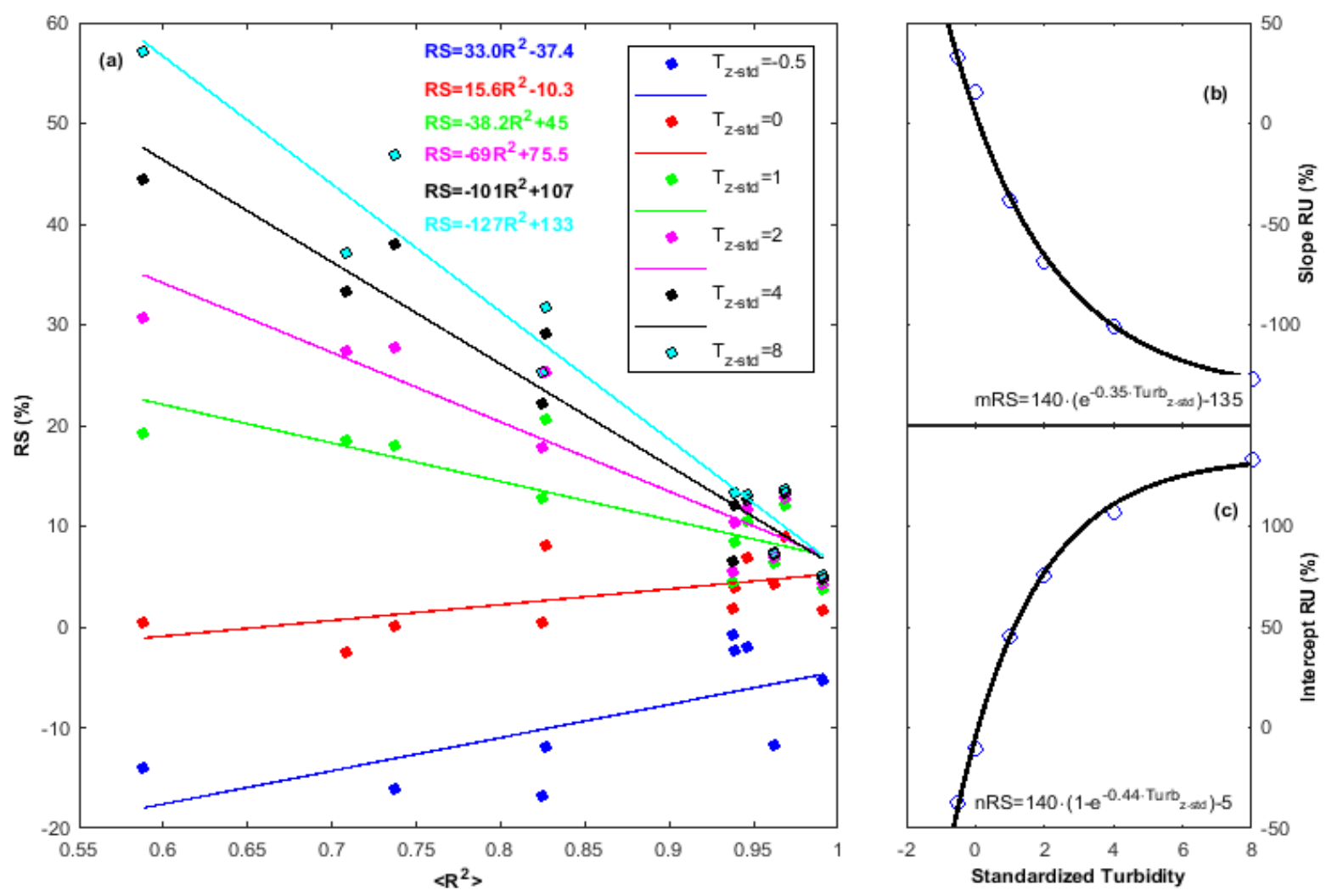

Figure 12: (a) Regression spread (RS) versus $R^{2}$ for 11 sample-SPMC/turbidity data calibration sets. Coloured dots denote values for different standardized turbidities $T_{z \text {-std }}$ (see section 3.4.2), coloured lines the corresponding results of the Eigenvalue regressions. (b) Slopes of the regression functions shown in panel (a) versus $T_{z-s t d}$. The line shows the result of the exponential fit. (c) The same as (b) for the intercepts. 
Version: August 11, 2019;

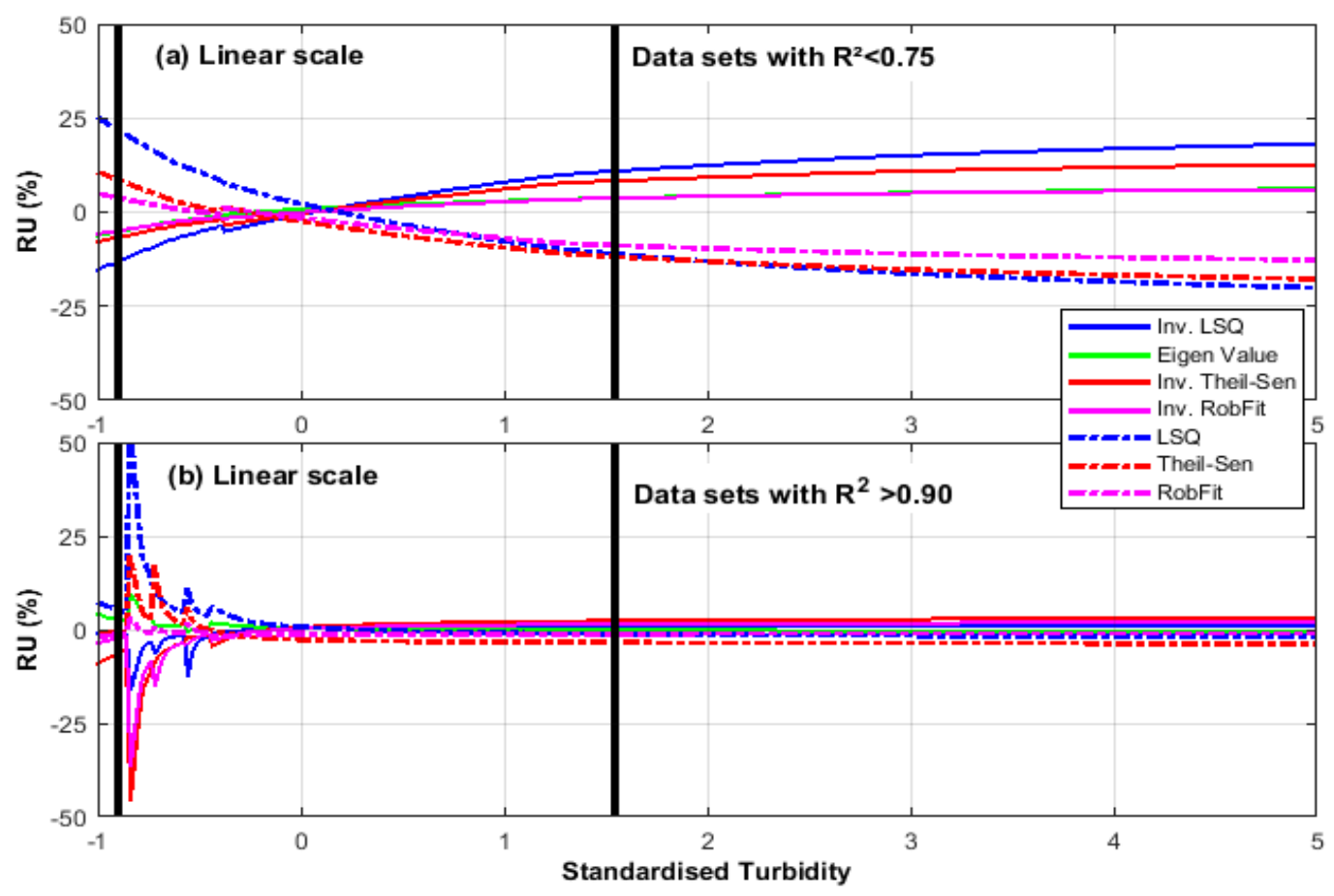

Figure 13: Regression model choice uncertainty $(R U)$ for the different regression models as a function of standardized turbidity $\left(T_{z-s t d}\right)$ for the linear scale. The examples show $R U$ averaged over data sets with $R^{2}<0.75$ (upper panels) and $R^{2}>0.90$ (lower panels). The black lines represent the 10 and $90 \%$ percentiles of $T_{z \text {-std }}$ averaged over all calibration data sets. Note the different limits in the ordinates of the left and the right panels. 


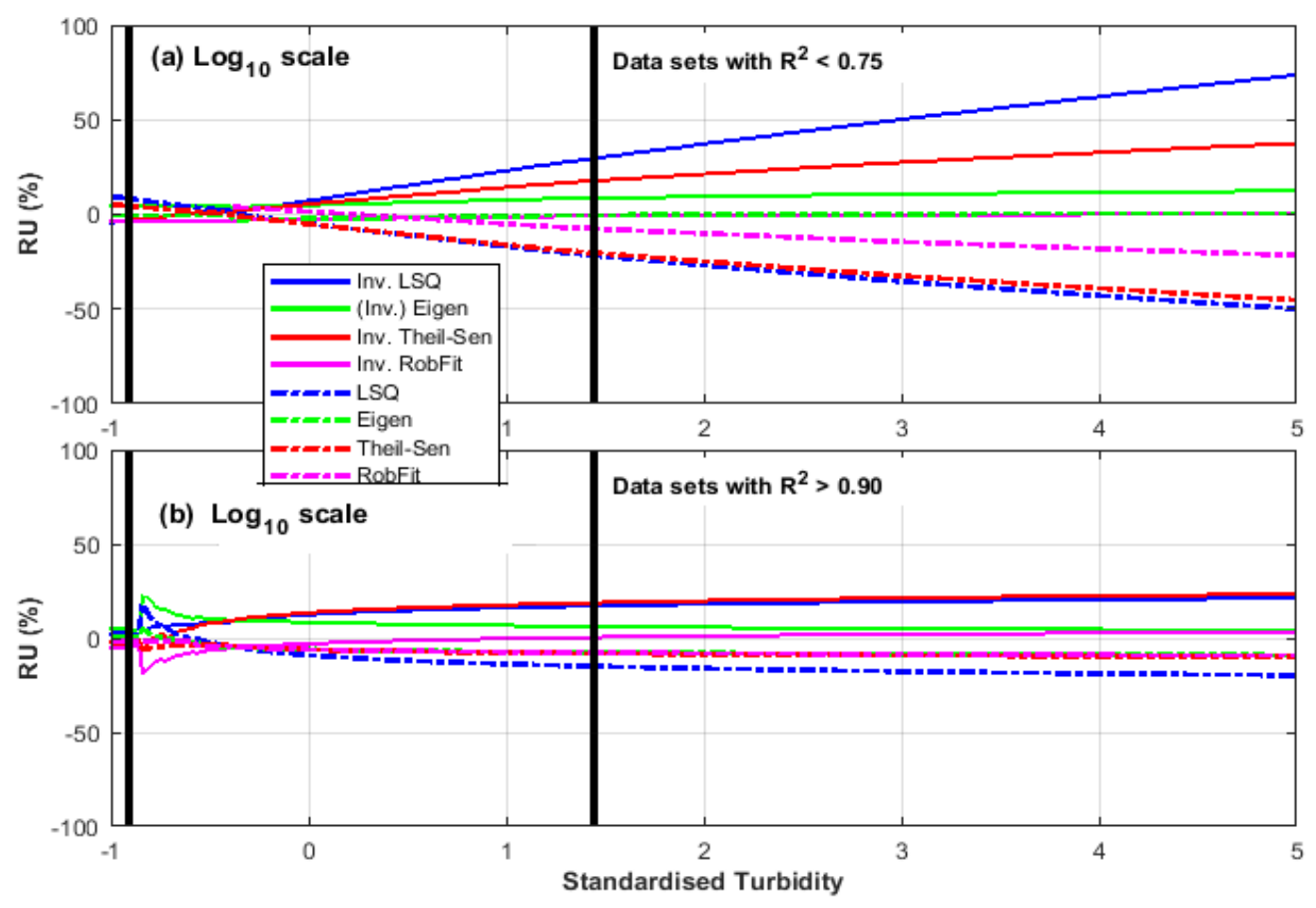

Figure 14: idem Figure 13 but now for the log10 - transformed data. 
Version: August 11, 2019;

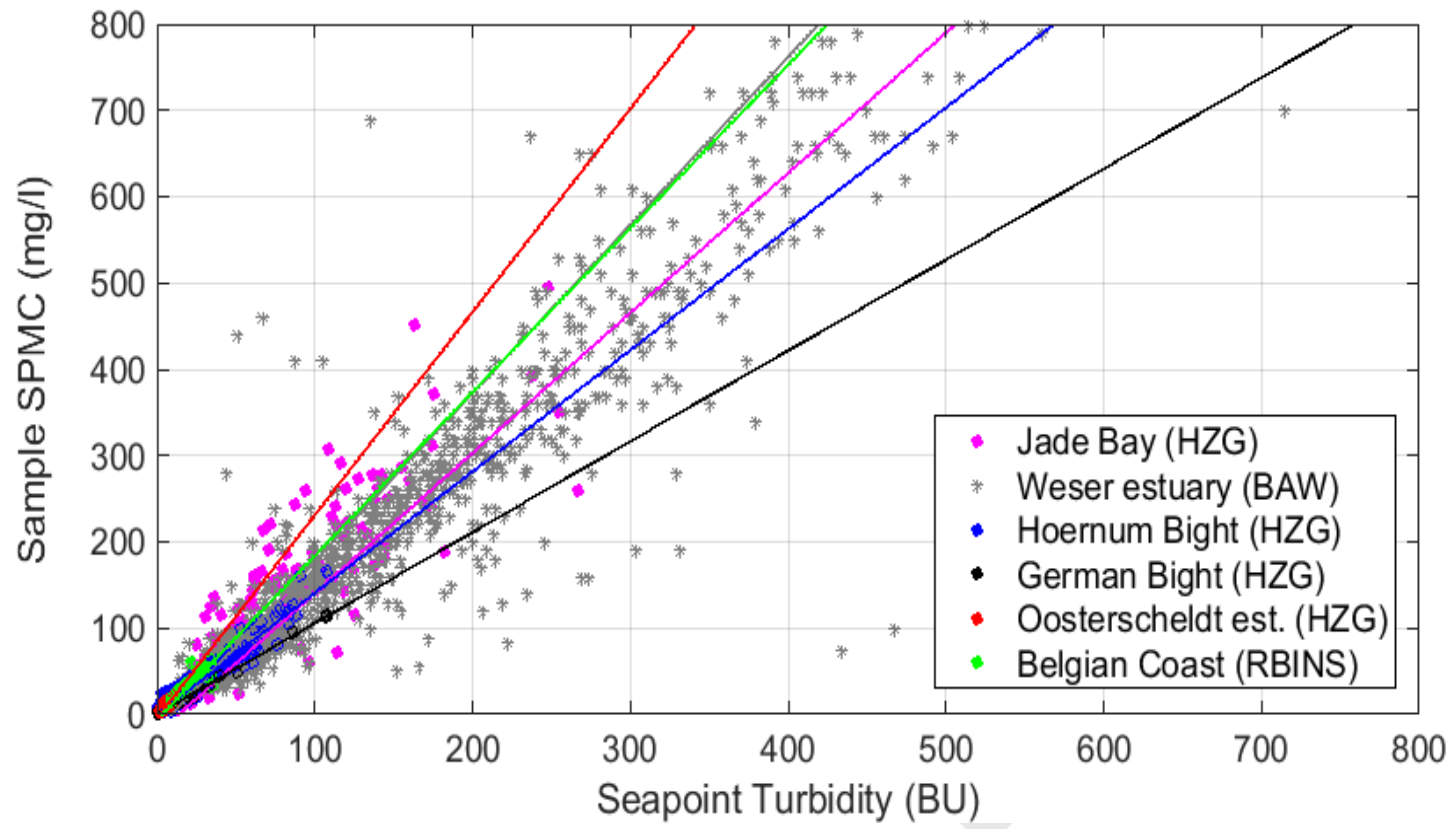

Figure 15: Sample SPMC as a function of Seapoint turbidity collected at various locations in the southern North Sea. The regression is calculated with the Eigenvalue model. The mean regressions are (SPMC $=a x+b)$ : all data $\left(R^{2}=0.88 ; a=1.53, b=-1\right)$; Jade Bay $\left(R^{2}=0.83 ; a=1.95, b=-\right.$ 17), Weser estuary $\left(R^{2}=0.83 ; a=1.62, b=-21\right)$; Hoernum Bight $\left(R^{2}=0.95 ; a=1.41, b=0\right)$; German Bight $\left(R^{2}=0.99, a=1.05, b=1\right) ;$ Oosterschelde estuary $\left(R^{2}=0.74 ; a=2.34, b=-3\right) ;$ Belgian coast (data from Figure 9, $R^{2}=0.61, a=1.90, b=-5$ ). 
Version: August 11, 2019;
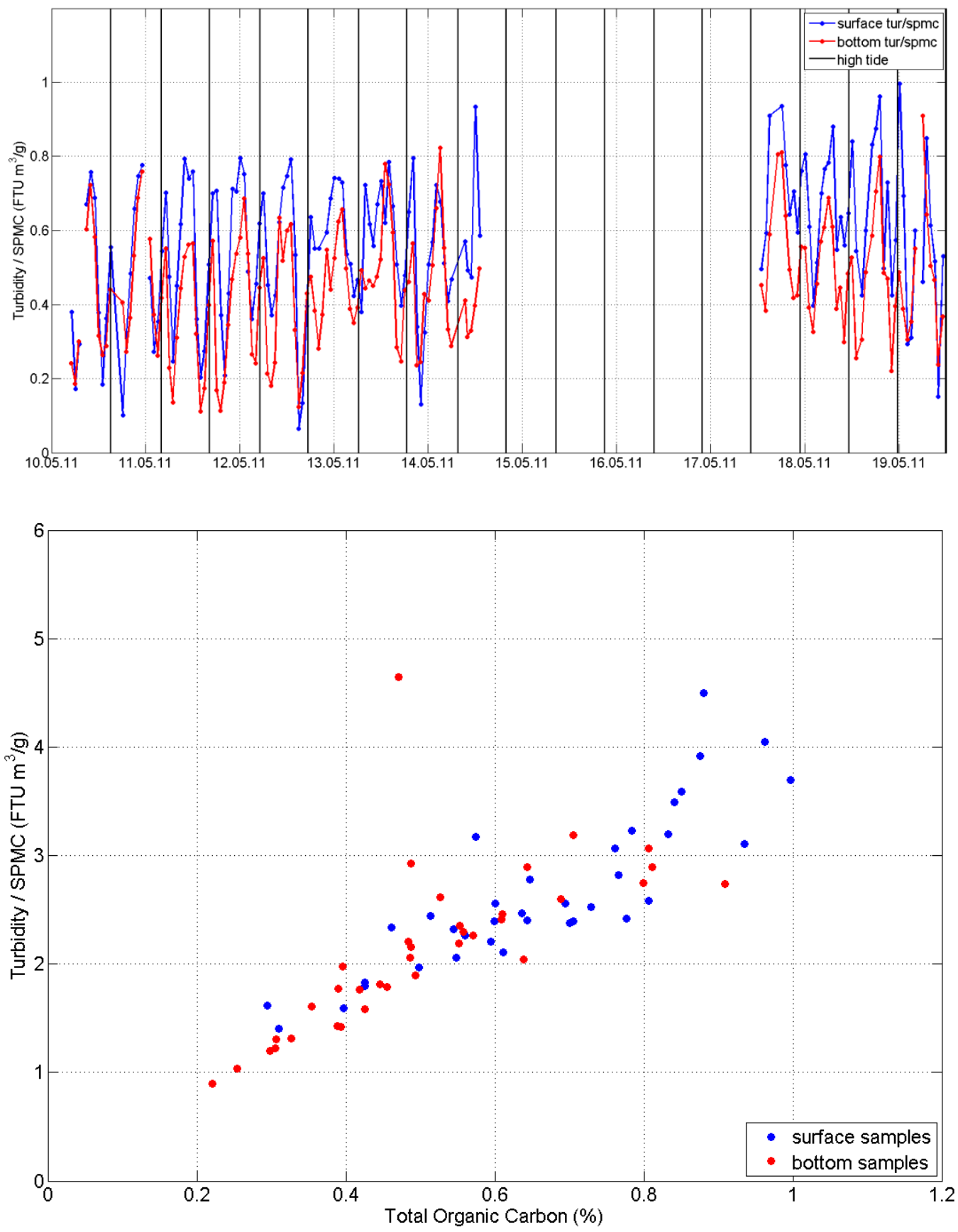

Figure 16: Sample SPMC and turbidity collected in the tidal inlet between two islands in the German Wadden Sea between 10 and 19 May 2011 (Becherer et al., 2016). Sample SPMC ranged between $7 \mathrm{mg} / \mathrm{l}$ and $500 \mathrm{mg} / \mathrm{l}$, during the storm (14-17/05) no samples have been taken. (a) Time series of the ratio turbidity/sample SPMC and (b) dependence of the specific backscatter $b=$ turbidity / sample SPMC on total organic carbon. All samples shown are taken after the storm, from $17^{\text {th }}$ through $19^{\text {th }}$ May. Surface data are taken $1 \mathrm{~m}$ below water surface. 


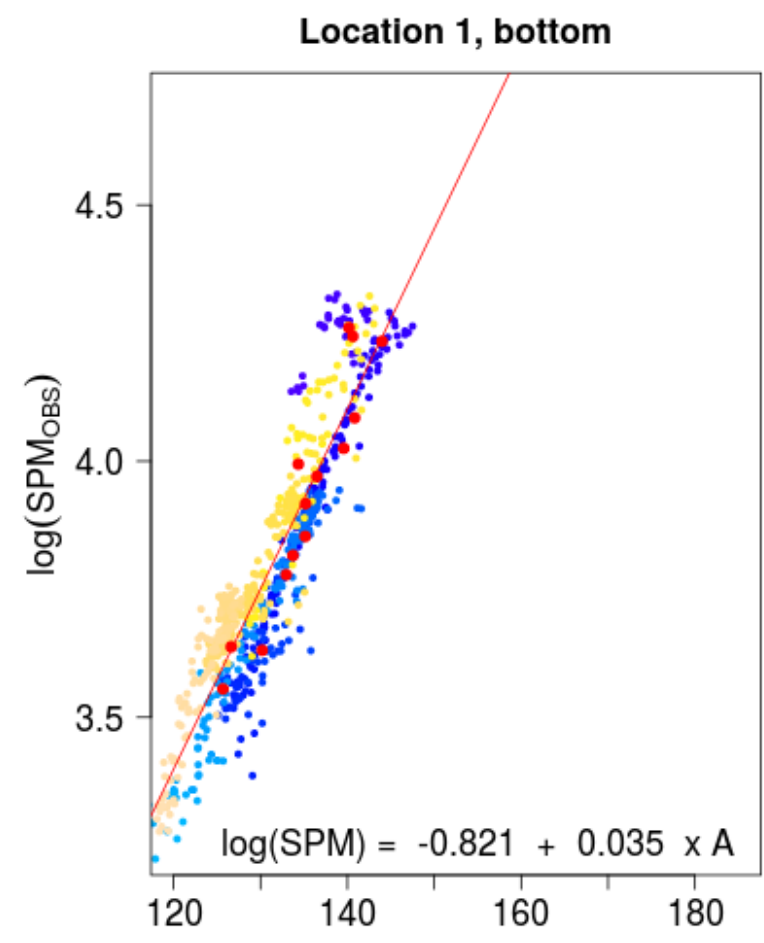

Acoustic backscatter amplitude (counts)

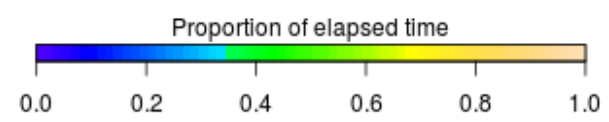

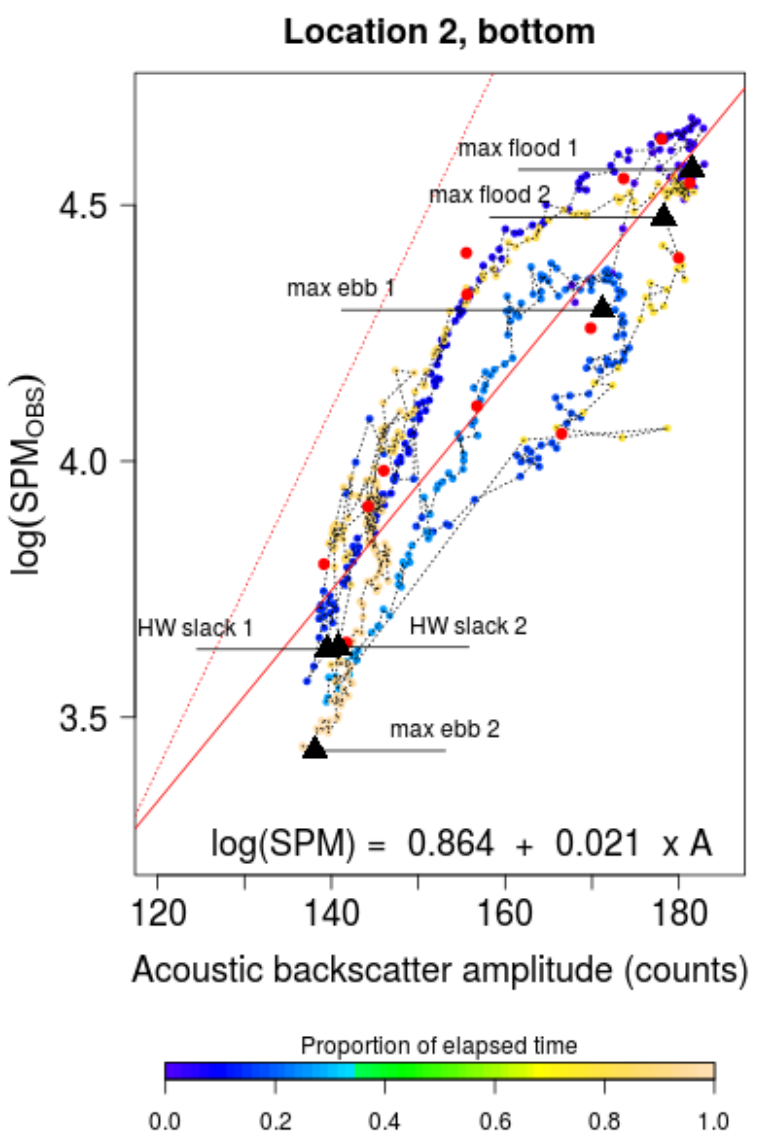

Figure 17: Relation between optical (YSI, type 6920 V2) and acoustical backscatter sensor (ADV (Nortek, 6MHz) at a muddy (location 1) and a sandy bed site (location 2) located 1200m apart in the main tidal channel of a brackish marsh located in the Scheldt estuary during 2 tidal cycles (Schwarz et al., 2017). 
Version: August 11, 2019;

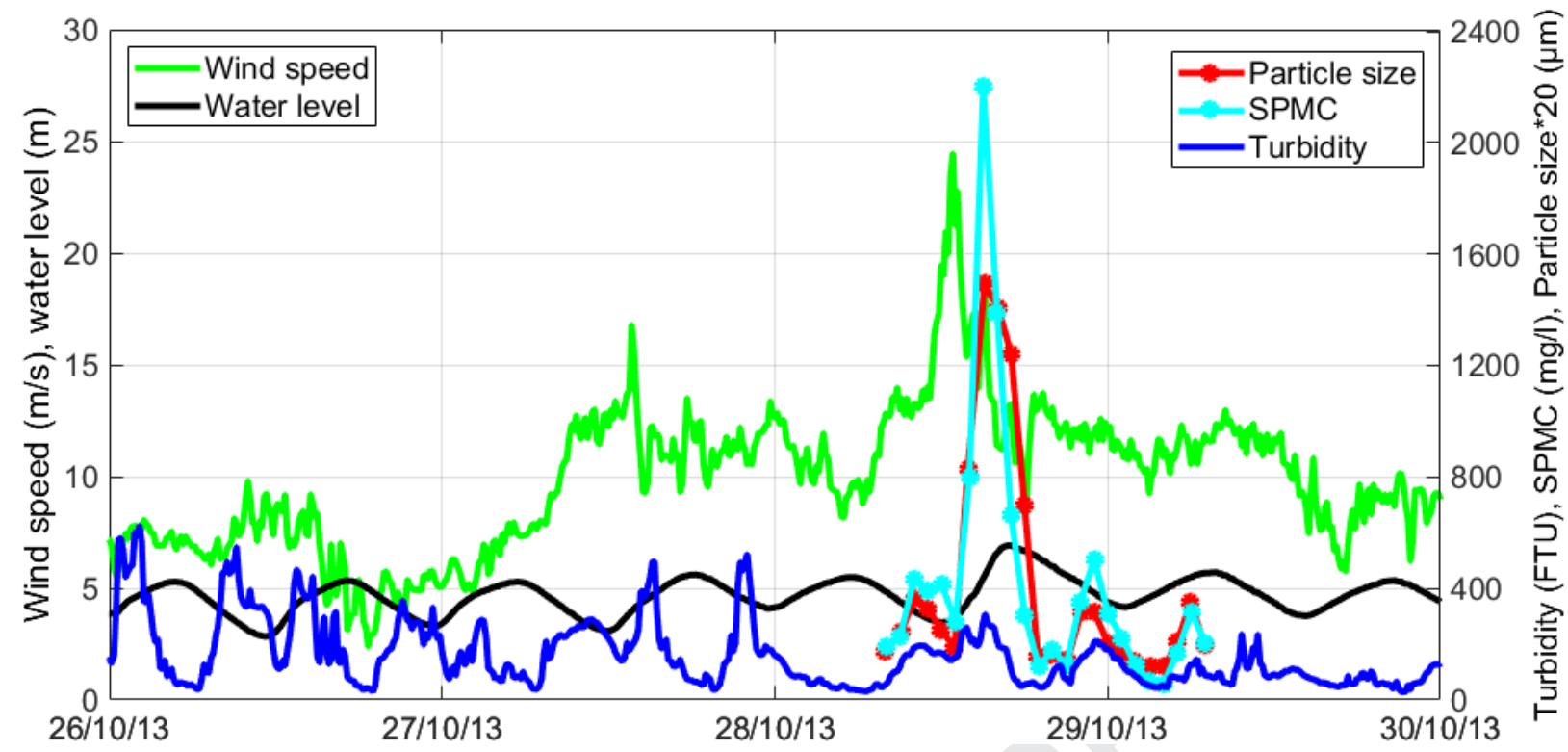

Figure 18: Changes in the ratio of turbidity to sample SPMC from 0.4 to 0.12 during a storm period in the Elbe estuary caused by the resuspension of sand grains. 
Version: August 11, 2019;

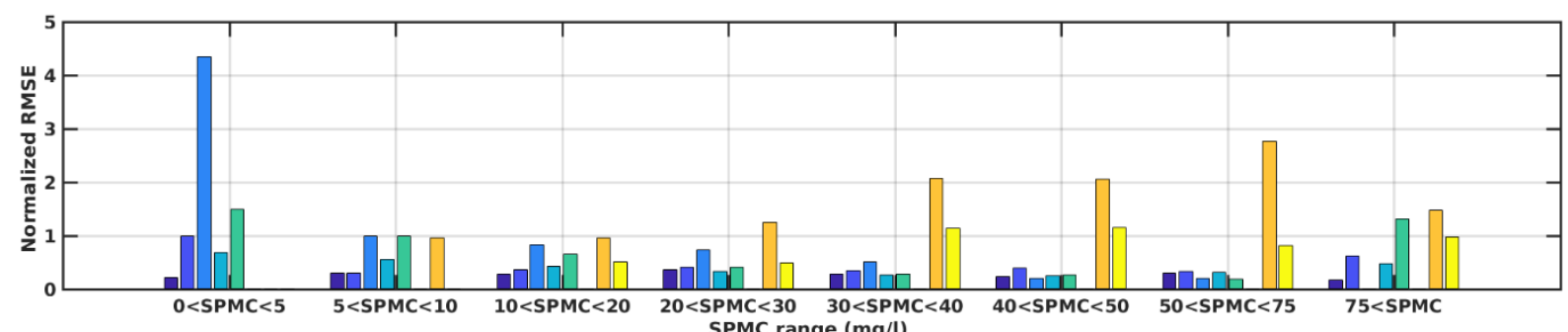
SPMC range ( $\mathrm{mg} / \mathrm{l})$

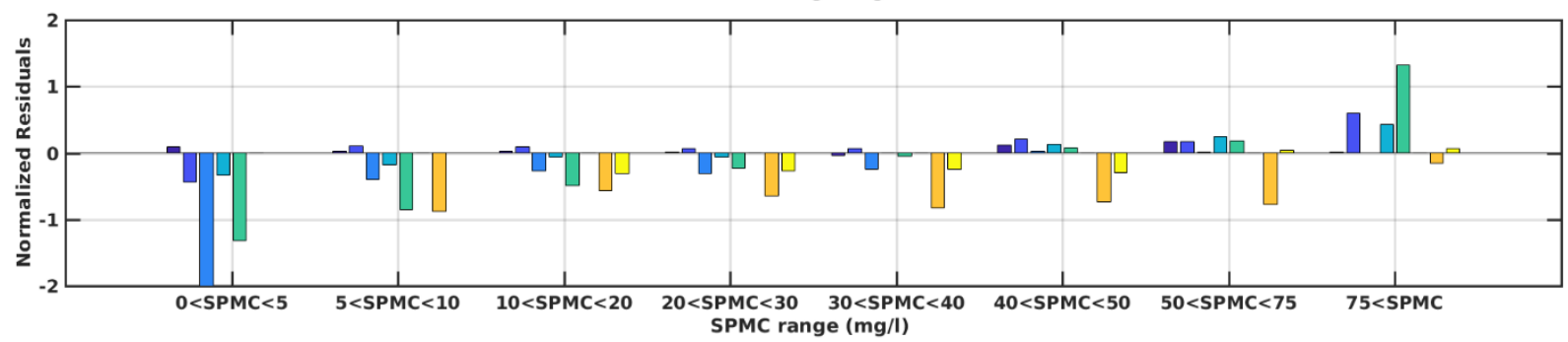

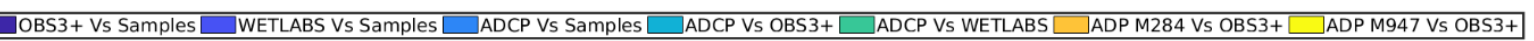

Figure 19: The model SPMC uncertainty (normalized RMSE and residuals) as a function of the reference SPMC (sample or sensor derived SPMC) and SPMC range (see the dataset shown in Figure 10 and 11) for an acoustical and some optical backscatter sensors. The reference SPMC is from sample or OBS3+ derived SPMC. 
Version: August 11, 2019;

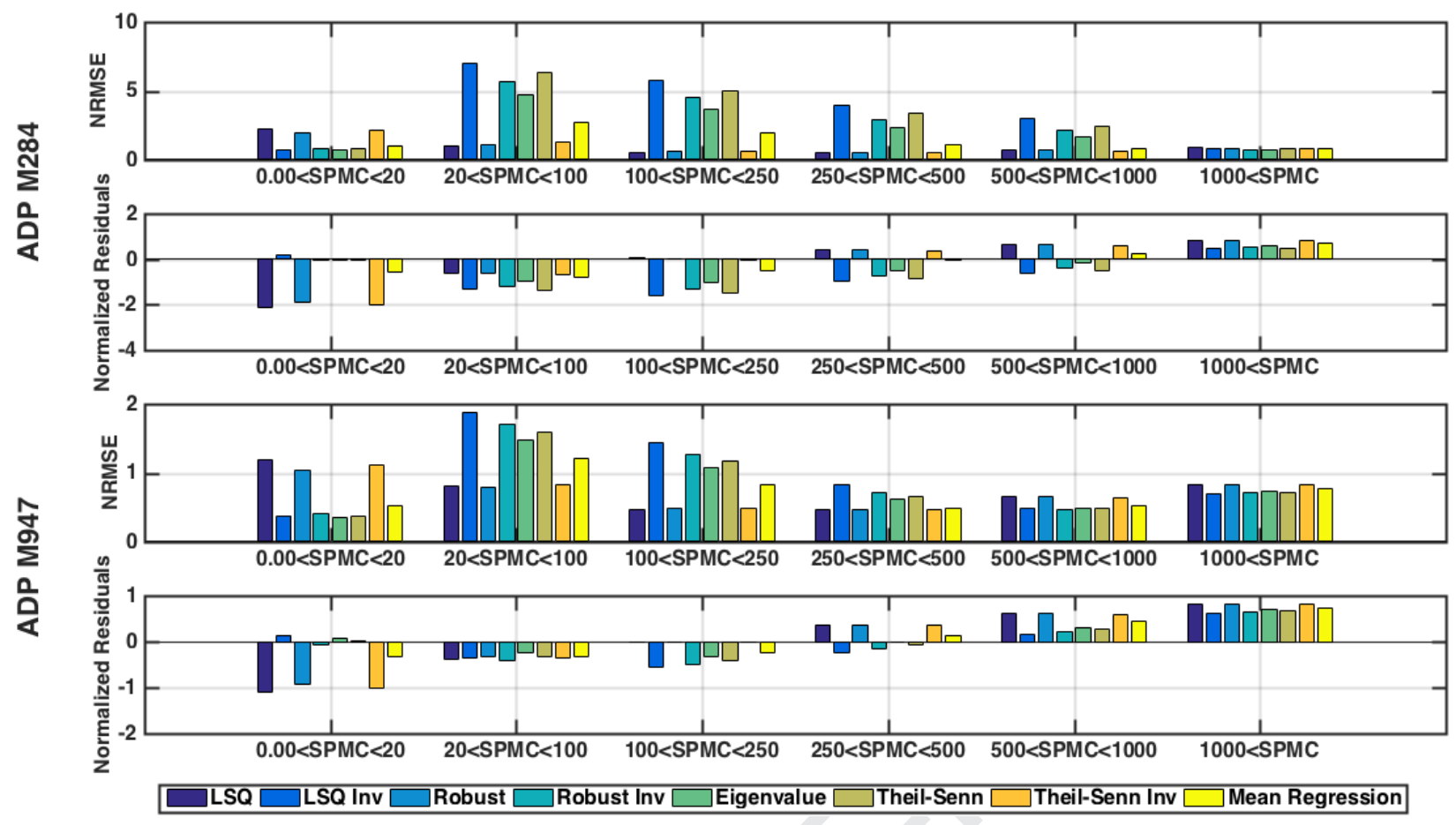

Figure 20: The model SPMC uncertainty for the ADP's of Figure 11 expressed as normalized RMSE and normalized residual for different regression models and as a function SPMC. Negative residuals have a higher ADP-derived SPMC than the OBS-derived one. 


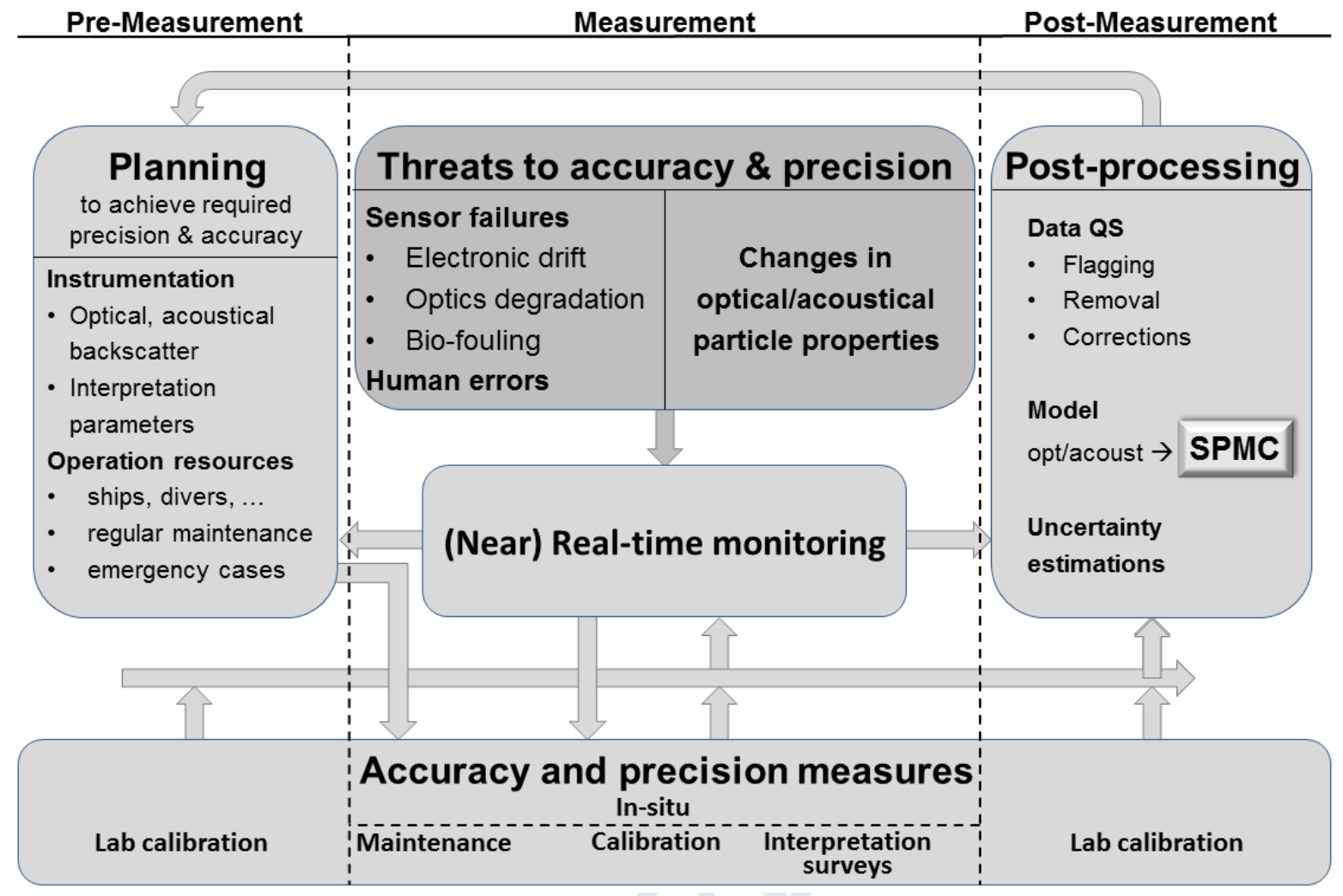

Figure 21: Workflow of activities and tasks to be performed in long-term SPMC measurements. The arrows indicate the flow information between the tasks and activities over the measurement phases. The measurement phases are plotted in serial order, but may overlap in the course of long-term installations. 
Version: August 11, 2019;

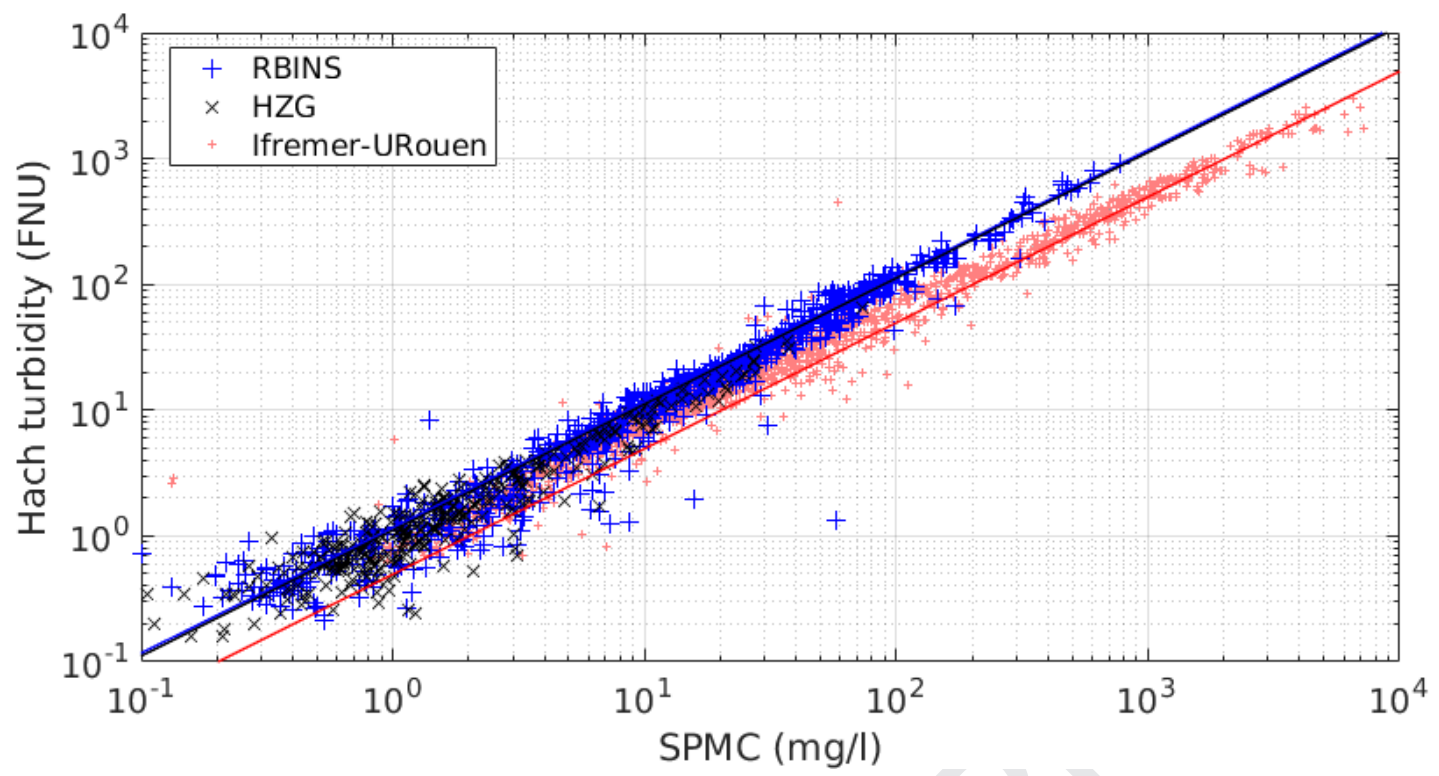

Figure 22: Scatterplot of sample SPMC ( $\mathrm{mg} / \mathrm{l})$ versus turbidity measurements from various waters around the world. The turbidity data from RBINS and HZG have been collected with a Hach 2100 S and those from Ifremer and University of Rouen by a Hach 2100N IS. HZG measurements were collected from 2009 to 2013, RBINS data are from 2007 to 2015 and Ifremer and University of Rouen data from 2015-2016. 

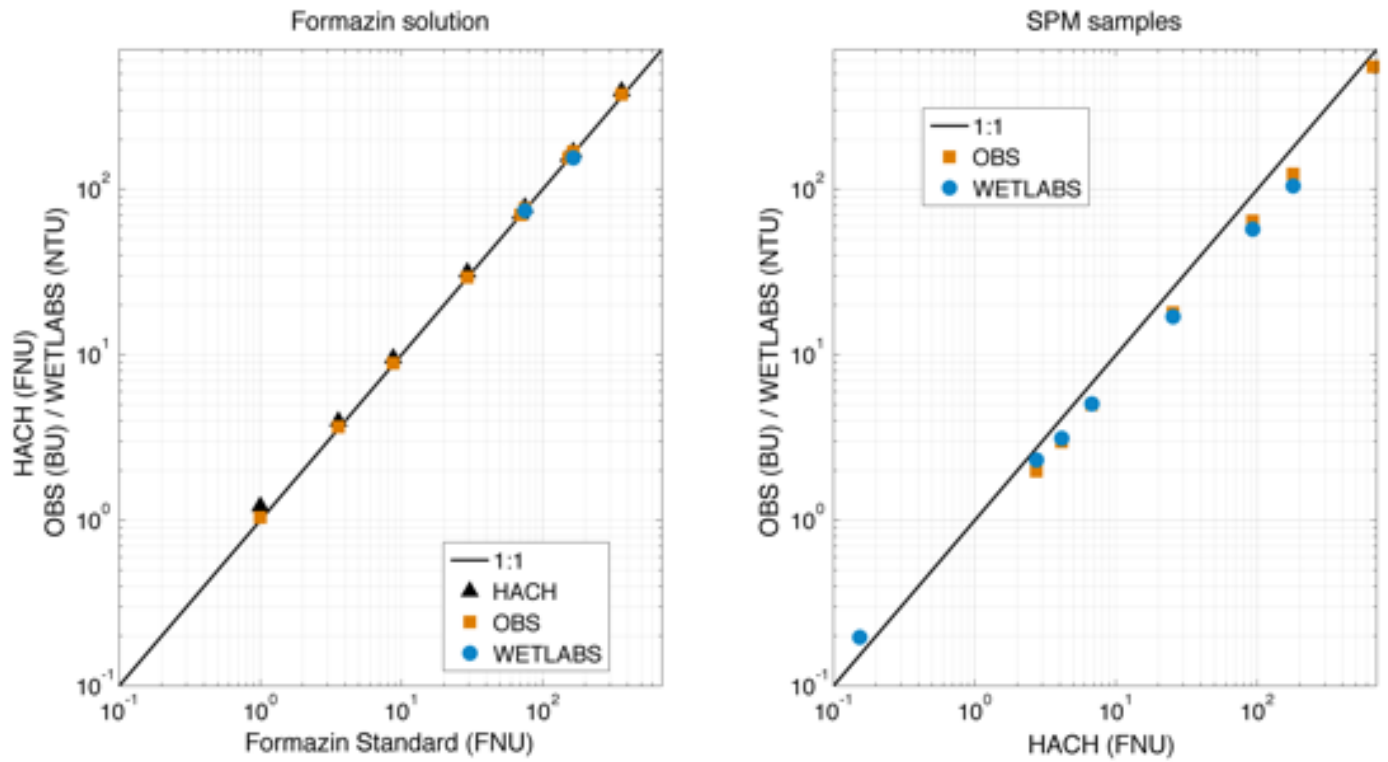

Figure 23: The performance of different sensors (side-scattering: Hach; backscattering: OBS3+ and Wetlabs) during sensor calibration with different formazine solutions (left) and as compared with natural SPM samples. 
Version: August 11, 2019;

Highlights

Errors associated with optical and acoustical backscatter sensors for SPMC are quantified A strict protocol limits the uncertainties

Systematic errors related to sensors, environment, sampling, calibration and humans may reach up to $\pm 20 \%$ and to biofouling to $100 \%$ or more.

Changes of the inherent particle properties may result in uncertainties up to $200 \%$. Additional sensors that detect particle properties are needed to limit uncertainties.

A model based on the $\mathrm{R}^{2}$ quantifies the uncertainty of the sensor derived SPMC in the calibration range and outside of it.

Hach-turbidities are a cheap alternative for sample SPMC within $20 \%$ uncertainty 